\title{
Coherent Control of Biomolecules
}


Samenstelling promotiecommissie:

Prof. dr. ing. M. Wessling Universiteit Twente, Enschede, Nederland

Prof. dr. J.L Herek Universiteit Twente, Enschede, Nederland

Prof. dr. M. Motzkus Philipps-Universität, Marburg, Duitsland

Prof. dr. H.J. Bakker FOM-Instituut AMOLF, Amsterdam, Nederland

Dr. J.T.M. Kennis Vrije Universiteit, Amsterdam, Nederland

Prof. dr. K.-J. Boller Universiteit Twente, Enschede, Nederland

Prof. dr. V. Subramaniam

Copyright (C) 2008 by J. Savolainen

The work described in this thesis was performed at the FOM-Institute for Atomic and Molecular Physics (AMOLF), Kruislaan 407, 1098 SJ Amsterdam. The Netherlands. The work is part of the research programme of the Stichting Fundamenteel Onderzoek der Materie (FOM), which is financially supported by the Nederlandse Organisatie voor Wetenschappelijk Onderzoek (NWO).

All rights reserved. No part of the material protected by this copyright notice may be reproduced or utilized in any form or by any means, electronic or mechanical, including photocopying, recording or by any information storage and retrieval system, without the prior permission of the author.

Cover design: Jenni Ahtiainen

ISBN 978-90-365-2671-5 


\section{COHERENT CONTROL OF BIOMOLECULES}

\section{PROEFSCHRIFT}

ter verkrijging van

de graad van doctor aan de Universiteit Twente, op gezag van de rector magnificus prof. dr. W.H.M Zijm, voorzitter van het College voor Promoties, in het openbaar te verdedigen op vrijdag 16 mei 2008 om 13.15 uur

door

Janne Savolainen

geboren op 3 november 1971

te Bern, Zwitserland 
Dit proefschrift is goedgekeurd door de promotor:

Prof. dr. J.L. Herek 
äidille ja isälle,

e para minha fofinha. 
Publications covered in this thesis:

Fanciulli, R., Willmes, L., Savolainen, J., van der Walle, P., Bäck, T., and Herek, J.L., Evolution Strategies for Laser Pulse Compression, 8th International Conference on Artificial Evolution, 4926 (2007).

Savolainen, J., Dijkhuizen, N., Fanciulli, R., Liddell, P.A., Gust, D., Moore, T.A., Moore, A.L., Hauer, J., Buckup, T., Motzkus, M., and Herek, J.L., Ultrafast energy transfer dynamics of a bioinspired dyad molecule, Journal of Physical Chemistry B, 112 (2008) 2678-2685.

Savolainen, J., Fanciulli, R., Dijkhuizen, N., Moore, A.L., Hauer, J., Buckup, T., Motzkus, M., and Herek, J.L., Controlling the efficiency of an artificial lightharvesting complex, PNAS, (2008) Accepted for publication.

Savolainen, J., van der Linden, D., Dijkhuizen, N., and Herek, J.L., Characterizing the functional dynamics of zinc phthalocyanine from femtoseconds to nanoseconds, Journal of Photochemistry and Photobiology A: Chemistry, 196 (2008) 99-105.

Savolainen, J., and Herek, J.L., Coherent control of the efficiency of a photodrug, in preparation.

Savolainen, J., Hauer, J., Buckup, T., Serrat, C., Motzkus, M., and Herek, J.L., Openloop coherent control of an artificial light-harvesting complex, in preparation.

Savolainen, J., van der Walle P., and Herek, J.L., Retracing the pathways of evolutionary algorithms, in preparation.

Other publications by J. Savolainen:

Pijpers, J.J.H., Hendry, E., Milder, M.T.W., Fanciulli, R., Savolainen, J.Herek, J.L., Vanmaekelbergh, D., Ruhman, S., Mocatta, D., Oron, D., Aharoni, A., Banin, U., and Bonn, M., Carrier multiplication and its reduction by photodoping in colloidal InAs quantum dots, Journal of Physical Chemistry C, 111 (2007) 4146-4152.

Buckup, T., Savolainen, J., Wohlleben, W., Herek, J.L., Hashimoto, H., Correia, R.R.B., and Motzkus, M., Pump-probe and pump-deplete-probe spectroscopies on carotenoids with $\mathrm{N}=9-15$ conjugated bonds, The Journal of Chemical Physics, 125 (2006) 194505-7.

Buckup, T., Wohlleben, W., Savolainen, J., Heinz, B., Hashimoto, H., Cogdell, R.J., Herek, J.L., and Motzkus, M., Energy flow in carotenoids, studied with pumpdeplete-probe, multiphoton- and coherent control spectroscopy, Ultrafast Phenomena XIV, (2005) 368-370. 
Buzady, A., Savolainen, J., Erostyak, J., Myllyperkiö, P., Somogyi, B., and KorppiTommola, J., Femtosecond transient absorption study of the dynamics of acrylodan in solution and attached to human serum albumin, Journal of Physical Chemistry B, 107 (2003) 1208-1214.

Hytönen, V.P., Laitinen, O.H., Grapputo, A., Kettunen, A., Savolainen, J., Kalkkinen, N., Marttila, A.T., Nordlund, H.R., Nyholm, T.K.M., Paganelli, G., and Kulomaa, M.S., Characterization of poultry egg-white avidins and their potential as a tool in pretargeting cancer treatment, Biochemical Journal, 372 (2003) 219-225.

Nordlund, H.R., Hytönen, V.P., Laitinen, O.H., Uotila, S.T.H., Niskanen, E.A., Savolainen, J., Porkka, E., and Kulomaa, M.S., Introduction of histidine residues into avidin subunit interfaces allows $\mathrm{pH}$-dependent regulation of quaternary structure and biotin binding, FEBS Letters, 555 (2003) 449-454.

Laitinen, O.H., Nordlund, H.R., Hytönen, V.P., Uotila, S.T.H., Marttila, A.T., Savolainen, J., Airenne, K.J., Livnah, O., Bayer, E.A., Wilchek, M., and Kulomaa, M.S., Journal of Biological Chemistry, 278 (2003) 4010-4014. 



\section{Contents}

1 Introduction 1

1.1 Dream of laser control . . . . . . . . . . . . . . . . . 1

1.1.1 Coherent control . . . . . . . . . . . . . . 2

1.1.2 Teaching lasers to control chemistry . . . . . . . . . 3

1.2 Biomolecular control . . . . . . . . . . . . . . . 4

1.2.1 Artificial light harvesting . . . . . . . . . . . . 5 5

1.2.2 Photodynamic therapy . . . . . . . . . . . . 6

1.3 Thesis context and overview . . . . . . . . . . . . . 7

\section{Methodology 9}

2 Learning loop and beyond $\quad 11$

2.1 Pulse shaping . . . . . . . . . . . . . . . 13

2.2 Transient absorption spectroscopy . . . . . . . . . . . . . . . . 15

2.2.1 Linear absorption . . . . . . . . . . . . . . . . . . 15

2.2.2 Transient absorption . . . . . . . . . . . . . . 16

2.2.3 Transient spectrum and molecular feedback . . . . . . . . 17

2.3 Evolutionary algorithms . . . . . . . . . . . . . . . 18

2.3.1 Characteristics of evolutionary algorithms . . . . . . . . . 19

2.3.2 Used evolution strategies . . . . . . . . . . . . . . . . . 20

2.3.3 Physical example . . . . . . . . . . . . . . 22

2.4 Challenges of black-box experiment . . . . . . . . . . 23

2.5 Outside the box . . . . . . . . . . . . . . 25

2.5.1 Pulse characterisation . . . . . . . . . . . . . . 25

2.5.2 Analysing search . . . . . . . . . . . . . . . . 25

2.5.3 Reducing search space . . . . . . . . . . . . . 26

2.5.4 Open-loop control . . . . . . . . . . . . . . . . 26

2.6 Quantum control spectroscopy ................ 27 
3 Experimental setup $\quad 29$

3.1 Transient absorption setup . . . . . . . . . . . . 30

3.2 Laser system . . . . . . . . . . . . . . . . . . 31

3.3 Noncollinear optical parametric amplifier . . . . . . . . . . . . . 31

3.4 Delay line . . . . . . . . . . . . . . . . . 32

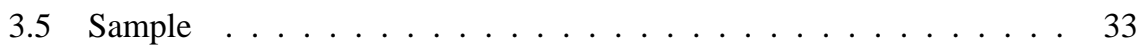

3.6 Detection system . . . . . . . . . . . . . . 33

3.7 Femtosecond pulse shaper . . . . . . . . . . . . . . . . . 34

3.7.1 Calibration ..................... 35

3.8 Pulse characterisation . . . . . . . . . . . . . . 36

4 Evolution strategies for laser pulse compression 39

4.1 Introduction . . . . . . . . . . . . . . . . . 40

4.2 Second harmonic generation as fitness function . . . . . . . . . . . 40

4.3 Introduction to optical second harmonic generation . . . . . . . . . . 41

4.3.1 Conceptual approach . . . . . . . . . . . . . . . . . 41

4.3.2 Mathematical approach . . . . . . . . . . . . 41

4.4 Role of the phase . . . . . . . . . . . . . . . . . . 42

4.5 Fitness and free parameters . . . . . . . . . . . . . . 42

4.6 Properties of fitness function, search landscape, noise . . . . . . . . . 43

4.7 Evolution strategies . . . . . . . . . . . . . . . . . . 43

4.7.1 Handling box constraints . . . . . . . . . . . . . . 44

4.7.2 Derandomised adaptation . . . . . . . . . . . . 46

4.7.3 Covariance matrix adaptation . . . . . . . . . . . . . . 46

4.8 Second harmonic generation: Simulations . . . . . . . . . . . . . 46

4.9 Second harmonic generation: Laboratory . . . . . . . . . . . . . . 48

4.10 Conclusions and outlook . . . . . . . . . . . . . . . . 48

5 Retracing pathways of evolutionary algorithms $\mathbf{5 3}$

5.1 Introduction . . . . . . . . . . . . . . . . . . . 54

5.2 Experimental .......................... 56

5.3 Results and discussion . . . . . . . . . . . . . . 57

5.3.1 Energy transfer yield .................... 57

5.3 .2 Emission yield . . . . . . . . . . . . . 60

5.4 Conclusions ........................ 61

\section{Applications $\quad 63$}

6 Ultrafast energy transfer dynamics of a bioinspired dyad molecule $\quad \mathbf{6 5}$

6.1 Introduction . . . . . . . . . . . . . . . 66

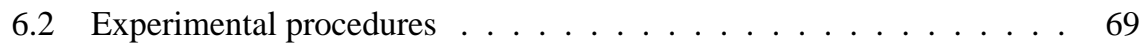

6.2.1 Data analysis ................. 70 
6.3 Results . . . . . . . . . . . . . . . . . 71

6.3.1 Steady-state measurements . . . . . . . . . . . . . 71

6.3.2 Time-resolved measurements . . . . . . . . . . . 71

6.3 .3 Oscillations .................... 74

6.4 Discussion . . . . . . . . . . . . . . . . . . 75

6.4.1 Target analysis . . . . . . . . . . . . . 75

6.4.2 Comparison: Natural versus artificial . . . . . . . . . 79

6.5 Conclusions .......................... 80

7 Controlling the efficiency of an artificial light-harvesting complex $\quad \mathbf{8 3}$

7.1 Introduction . . . . . . . . . . . . . . . . . 84

7.2 Materials and methods . . . . . . . . . . . . . 85

7.3 Results ....................... 86

7.4 Discussion . . . . . . . . . . . . . . . . . 88

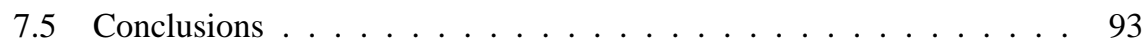

8 Characterising and controlling photodrug efficiency 95

8.1 Introduction . . . . . . . . . . . . . . . . . . 96

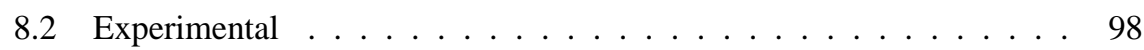

8.2.1 Data analysis . . . . . . . . . . . . . . . . . 99

8.3 Characterisation results . . . . . . . . . . . . . 100

8.3.1 Steady-state and fluorescence measurements . . . . . . . 100

8.3.2 Time-resolved spectroscopy . . . . . . . . . . . . . . 101

8.4 Discussion . . . . . . . . . . . . . . . . . . . 104

8.5 Coherent control results and discussion . . . . . . . . . . . 107

8.6 Conclusions and outlook . . . . . . . . . . . . . 111

$\begin{array}{ll}\text { A Appendix } & 113\end{array}$

$\begin{array}{ll}\text { Bibliography } & 117\end{array}$

$\begin{array}{ll}\text { Summary } & 131\end{array}$

$\begin{array}{ll}\text { Samenvatting } & 137\end{array}$

$\begin{array}{ll}\text { Resumo } & 139\end{array}$

$\begin{array}{ll}\text { Yhteenveto } & 141\end{array}$

$\begin{array}{ll}\text { Acknowledgements } & 143\end{array}$ 



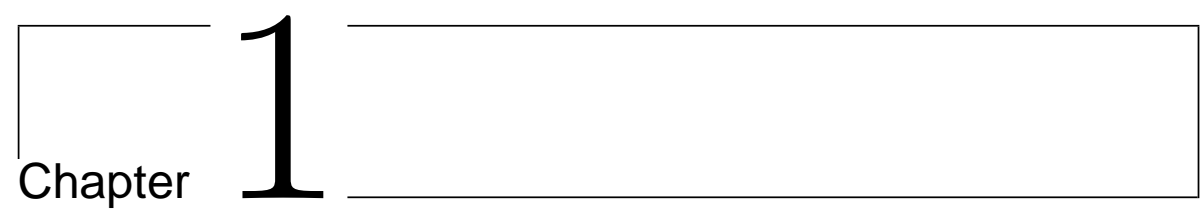

\section{Introduction}

\subsection{Dream of laser control}

Ever since the discovery of the laser in 1960, the dream of using the characteristics of laser light to control chemical reactivity has fascinated scientists. The ultimate goal of chemistry is as old as chemical synthesis itself: Selectivity. That is, to maximise the yield of desired products and suppress formation of unwanted byproducts. Traditionally, the approach to chemical control has been passive, using macroscopic quantities like temperature and pressure to influence the yields of reactions, in which wanted and unwanted products are formed statistically according to the governing potential energy landscapes. In other words, in passive control the environment (i.e. the energy landscape) of a reaction is manipulated and the reaction yields vary accordingly. The fundamental difference with the dream of laser control is the aim for active control where chemical and physical processes are manipulated coherently using the microscopic properties of the light-matter interaction. To compare:

Passive control: Reactant molecules and any surrounding solvent molecules are not subjected to manipulation by external influences during the evolution from reactants to products. Further, the evolution of energised reactants is largely or completely incoherent. Finally, the traditional role of the experimenter is merely to initiate the reaction without having any control of subsequent evolution of the system. Typical passive control knobs are concentration, temperature, pressure, $\mathrm{pH}$, solvent, catalyst, synthetic design of reactants, etc.

Active control: Molecular dynamics are manipulated externally to influence the evolution of the reactant molecule to generate more or all of a particular product. The control knobs of active control relate to the used control field and its intensity, phase, polarisation, spectral content, time profile, etc.

The first attempts of using optical fields of lasers to control chemistry focused on bond-selective (mode-selective) reactivity by laser light. Conceptually, the idea is simple: To choose the light frequency to be in resonance with the vibrational frequency 
of the bond to be broken. The resonant activation then accumulates energy into the vibrational mode and leads to selective dissociation. However, in practice the motion belonging to the chosen vibrational mode is coupled to many atoms within the molecule and intramolecular vibrational redistribution leads to a loss of selective excitation and 'heating' of the molecule. Hence, this simple approach works only for a limited number of small prototype molecules $[1,2]$.

\subsubsection{Coherent control}

To overcome this hurdle, we need to consider the quantum mechanical nature of matter and make use of the coherence properties of laser light in controlling the reactions. In other words, we seek coherent control which means control by exploiting a broad range of quantum interference effects and utilising the relevant properties of the molecular Hamiltonian. Coherent control can be realised using different feasible schemes in time domain, frequency domain, and adiabatic domain.

The multiple path interference control scheme works in the frequency domain. According to the scheme, the probability of forming a product depends on the relative phase of two continuous-wave laser fields tuned to interfering excitation pathways, for example one-photon and three-photon excitation [3]. Briefly, we can postulate that the solutions of the Schrödinger equation (as a function of energy) provide a complete description of the system, and that the solutions to the time-dependent Schrödinger equation can be represented as a superposition of the time-independent eigenstates. Therefore, it is possible to uniquely correlate specific product state wave functions with the total system eigenfunctions. Now, if two independent pathways connect the same initial and final states, the probability of forming a specific final state is proportional to the square of the sum of amplitudes associated with the individual transitions from the initial to final states. Thus, depending on the relative phase between the two fields we have constructive or destructive interference in the probability of forming the product. In practice, this means that by controlling the phase difference between the excitation laser fields we can control the product yield.

The stimulated Raman adiabatic passage (STIRAP) scheme uses electric fields large enough to generate many so called Rabi oscillations. STIRAP uses a 'counterintuitive' pulse sequence where a Stokes pulse first creates a coherent superposition of two initially unpopulated states. When this coherent superposition state is coupled to a populated state by the pump pulse, a 'trapped' state is formed and the pump field cannot transfer population to the intermediate state [4].

The time domain control scheme uses femtosecond laser pulses because that is the time scale of nuclear motion in molecules. In the time domain pump-dump scheme, the reactant can form two or more different products on the ground state potential energy surface (PES) [5, 6]. An electronic excited state of a molecule has generally different conformation than the ground state such that the vibrational wavepacket created on the excited PES evolves by translation. Now, by proper timing of a suitable fs pulse we can interfere with the wavepacket evolution and dump the molecule at a particular moment to the desired ground-state product. Note that according to the second order perturbation theory the transfer of amplitude from excited to ground state is not sensitive to the relative phase of the pump and dump laser pulses. Further, the time 
and frequency tuning of the pump and dump pulses requires precise knowledge of the PESs of the electronic ground state and first electronic excited state.

The presented early strategies of coherent control have all the fundamental limitation that they require precise knowledge of the molecular Hamiltonian and hence work best on small model systems. We want to combine these schemes and utilise a broad range of quantum interferences to discriminate in the favour of desired final states over unwanted pathways when we manipulate the evolution of the system. Hence, we ask: With a given target distribution of photoproducts and the quantum mechanical equations of motion, what are the characteristics of the electric field required to guide the temporal evolution of the system appropriately? In other words, can we perform control field design to find the right settings for the control knobs of active control: intensity, phase, polarisation, spectral content, time profile, etc.

\section{Optimal control theory}

Optimal control theory (OCT) aims to find these control fields by using wavepacket (or density matrix) propagations between initial and desired final states in combination with an optimisation algorithm [7]. However, OCT has many inherent difficulties. First, it needs accurate PESs and molecular Hamiltonians as input. Polyatomic molecules are computationally heavy and PESs are rarely known, especially over all range of nuclear separations necessary to describe a reaction. Second, the BornOppenheimer approximation (which provides the basis for calculations) often fails at numerous points on the multidimensional space. Third, the multidimensional character of the PES ( $3 N-6$ for polyatomic molecules where $N$ is the number of atoms) makes the search space too large to be scanned completely. Finally, the predicted fields are often difficult to reproduce accurately enough under laboratory conditions.

\subsubsection{Teaching lasers to control chemistry}

To overcome the aforementioned difficulties, an experimental approach was proposed where femtosecond pulse shaping is steered by an optimisation algorithm and experimental molecular feedback in an iterative learning loop [8]. A schematic representation of this approach is shown in Figure 1.1. The approach takes a fundamental view to the problem by making use of the fact that a molecule knows its own Hamiltonian: For a given control field $E(t)$, the molecule solves its own the Schrödinger equation rapidly and the answer can be then read out by the experiment. By testing several pulse shapes on the sample and creating new pulse shapes based on the feedback the learning algorithm tries to find important features of the control field. The loop proceeds iteratively as better solutions are found.

The learning-loop approach offers several advantages. For the purposes of biomolecular control, the most important one is that no a priori knowledge of the Hamiltonian is necessary. This makes the learning-loop approach an appealing tool for systems that are intractable on the quantum mechanical level. The approach has proven broadly applicable as coherent control by learning-loop has been realised in several successful studies on complex systems. Examples of pioneering work include control over nuclear motion [9-14], electron dynamics [15] as well as nonlinear optical phenomena $[16,17]$. 


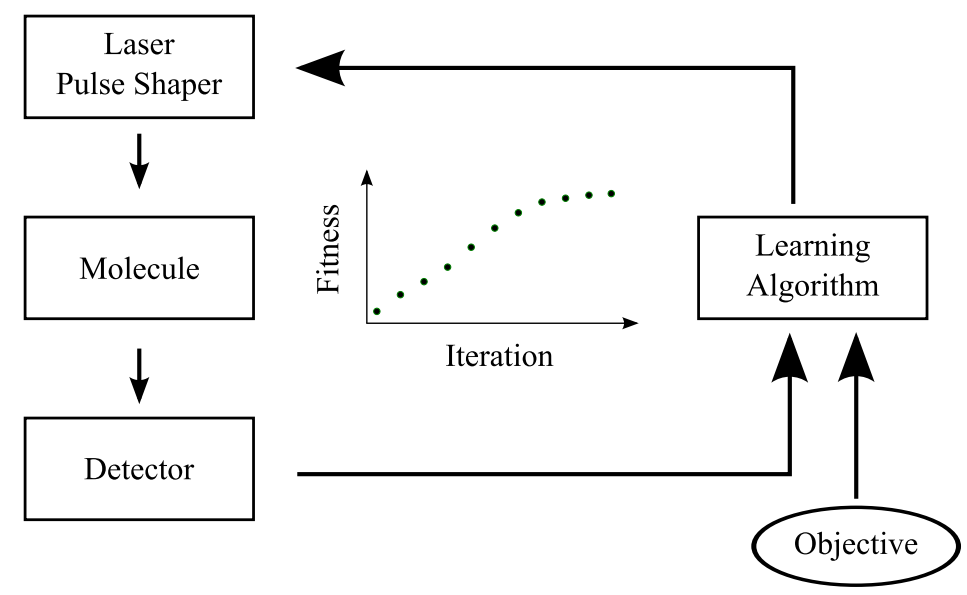

Figure 1.1: Teaching lasers to control molecules. Experimenter sets a control objective for a given molecular system. A learning algorithm steers laser pulse shaping in an iterative loop where new pulse shapes are created based on the feedback information from the molecule. A fitness function is evaluated based on the feedback. As the pulse shapes get better a learning curve shows the increase of the fitness.

In summary, coherent control of chemical reactions is a rapidly expanding field of science. As a versatile approach the learning loop experiments hold many promises for studies of various kinds of systems. The existing literature already offers a great deal of new knowledge on the properties of matter that is obtained using the learning loop. The field is rapidly developing and new leaps in understanding the obtained results and deciphering control mechanisms are appearing one after another as scientists refine methodology, cultivate control objectives and move further towards increasingly relevant and interesting molecular systems.

\subsection{Biomolecular control}

Biomolecules are here loosely defined as complex polyatomic molecules that perform specific biologically-relevant functions i.e. functional molecules. The structural and functional complexity of biomolecules presents a serious challenge for detailed spectroscopical studies. In this work we develop a novel approach for reducing biological complexity. Our strategy is to apply the tools of coherent control to study biomolecules in solution and especially make use of the versatility of the learning loop approach. Such optimal control experiments have a great potential to reveal new functionally relevant information, since finessed quantum mechanical manipulations can be used to unravel interactions that govern molecular dynamics. By analysing the pulse shapes we aim to identify functionally relevant motions as well as other microscopic properties that influence reactivity. In particular, we have applied this approach to explore:

- Emission properties of fluorescent label molecules 

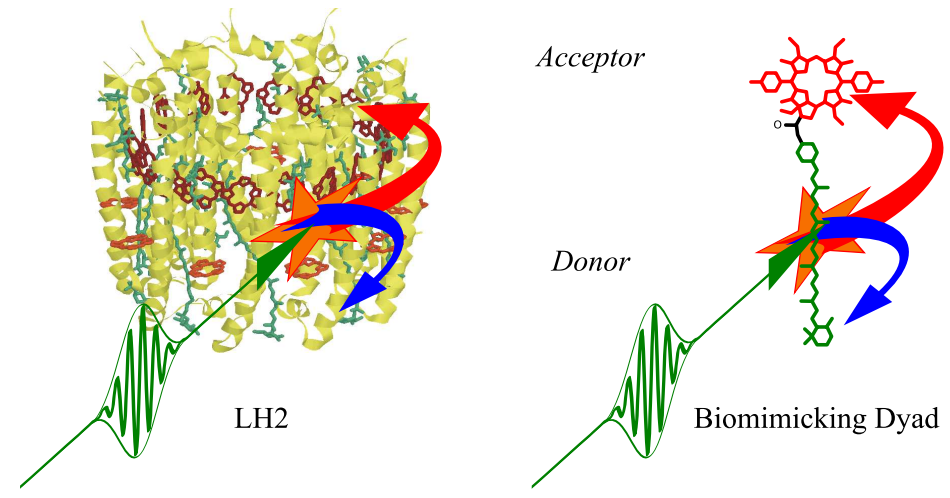

Figure 1.2: Energy transfer process in light harvesting. Left: The structure of the natural LH2 complex. Right: the structure of the artificial light harvesting dyad. Following photoexcitation by a laser pulse (green arrow) the excited donor molecules (green) are deactivated via competing pathways of energy transfer (red arrow) to acceptor molecules (red) and internal conversion (blue arrows).

- Energy-transfer pathways in a bioinspired artificial light-harvesting complex

- Efficiency of photoactive molecular switches

- Emission yield of silicon nanocrystals

- Efficiency of sensitiser molecules for photomedical applications

In this thesis we focus on two of these studies, as introduced below.

\subsubsection{Artificial light harvesting}

The first molecular application is on artificial light-harvesting and relates to one of the important challenges of science and technology today: Conversion of light energy into chemical potentials using artificial photosynthesis [18]. The design of such artificial systems takes inspiration from Nature and its complicated natural light-harvesting complexes. By using this idea of biomimicry the structural complexity can be reduced to the basic elements while the functionality is preserved [19].

In this thesis, we study an artificial light-harvesting complex that closely mimics the photophysics of the LH2 complex from the purple bacterium Rhodopseudomonas acidophila. The chosen system is a dyad molecule consisting of a single donor (carotenoid) and single acceptor (purpurin) moiety, thus reducing the structural complexity significantly compared to the natural complex. Figure 1.2 shows the structure of LH2 having several donor (green) and acceptor (red) molecules embedded in a protein matrix; alongside the structure of the dyad having only one of each covalently bound with an amide linker. In both systems the energy of blue-green light is first absorbed by a donor molecule and then passed on to the acceptor by an ultra-fast energy transfer process. Competing with the energy transfer (ET, red arrow) process, an internal conversion (IC, blue arrow) process leads to intramolecular deactivation and further to 


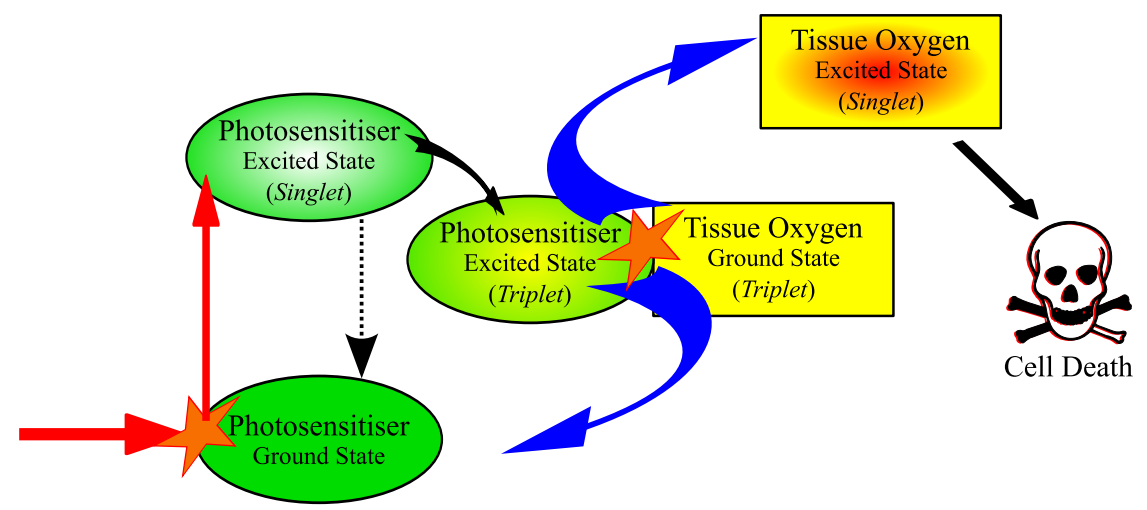

Figure 1.3: Schematic representation of energy-flow pathways of photodynamic therapy. Photoexcited sensitiser molecules trigger the formation of singlet oxygen, which then leads to cell death.

energy loss by heat dissipation. It is this branching between the functional (ET) and the loss (IC) channels that we seek to manipulate by means of coherent control. We first resolve the intricate details of the photophysics in an comprehensive characterisation study (Chapter 6) and then realise coherent control of energy flow in the dyad molecule using adaptive femtosecond pulse shaping (Chapter 7).

\subsubsection{Photodynamic therapy}

Photodynamic therapy (PDT) is a fairly novel and promising technique in cancer treatment [20], in which so-called photosensitiser molecules localise at tumour cells and, upon optical excitation, facilitate the production of singlet oxygen which is toxic to cancerous cells. Figure 1.3 shows a schematic representation of the PDT mechanism via the so-called type 2 sequence. Briefly, photosensitiser molecules are administered and they concentrate in cancerous tissue. The photosensitiser is excited by a light source from the ground singlet state to the first excited singlet state from which it undergoes intersystem crossing to the lowest lying excited triplet state. Oxygen is abundantly available in the tissue and has a ground triplet state. Therefore, as the photosensitiser and an oxygen molecule come in proximity, an energy transfer can take place where the photosensitiser to relaxes to its ground singlet state while oxygen becomes excited to its excited singlet state oxygen. Singlet oxygen is a very aggressive chemical species and will very rapidly react with any nearby biomolecules. Ultimately, these destructive reactions will result in cell death.

The PDT reaction chain offers challenges along the whole process starting from the synthesis of an optimal photosensitiser to delivery mechanisms localising the drug to the proliferating tissue and to providing selective uptake of photosensitisers to particular tissue layers, as well as to precise delivery of suitable light at the treatment site. From the photophysical viewpoint, the efficacy of this technique depends on the properties of the photosensitiser: the wavelength and extent of absorption, quantum 
yields of excited state processes as well as the photodegradation rate. Relatively little is known about the excited state properties and intramolecular dynamics of photosensitisers, and hence the molecular basis of the mechanisms underlying PDT is not well understood.

We chose zinc phthalocyanine (ZnPc) in this study to serve as a model photosensitiser. ZnPc has been in clinical trials of PDT, it produces singlet oxygen with high yield and has a high absorption cross section in the far-red part of the spectrum where human tissue is most penetrable. After characterising the system with techniques such as steady state absorption, fluorescence and transient absorption spectroscopies, we ask if it is possible to find pulse shapes that enhance the functional efficiency and minimises the energy dissipation vie the competing loss channels.

\subsection{Thesis context and overview}

Part I discusses the methodological aspects of coherent control of molecular processes. Chapter 2 (Learning loop and beyond) is a conceptual chapter on the used experimental approach and related techniques as well as on the challenges related to them. Chapter 3 (Experimental setup) dives deeper into the technical details of the used experimental setup. The following Chapters 4 (Evolution strategies for laser pulse compression) and and Chapter 5 (Retracing the pathways of evolutionary algorithms) present the novel results in the methodology development.

Part II presents the biomolecular applications on the chosen two target systems: an artificial light-harvesting complex and a photosensitiser for photomedical applications. In Chapter 6 (Ultrafast energy transfer dynamics of a bioinspired dyad molecule) the photophysics of the artificial light-harvesting complex are studied in detail and in Chapter 7 (Coherent control of an artificial light-harvesting complex) we show how the branching between the competing energy-flow pathways is manipulated by adaptive femtosecond pulse shaping as well as by open-loop quantum control spectroscopy. Photophysics of the model photosensitiser zinc phthalocyanine as well as results of coherent control of the triplet yield of the photosensitiser are presented in Chapter 8 (Characterising and controlling photodrug efficiency). 

Part I

Methodology 



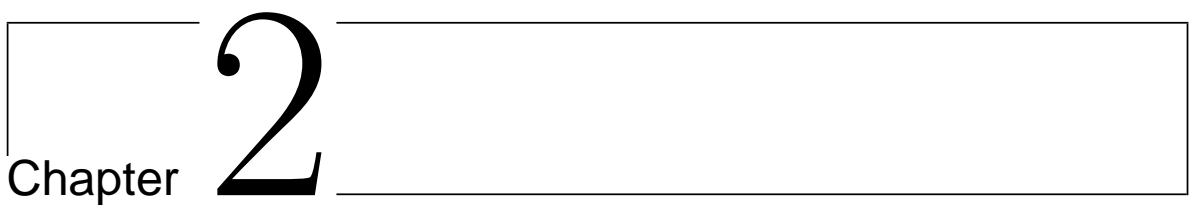

\section{Learning loop and beyond}

In this chapter we will take a closer look at the process of teaching lasers to control molecular reactions. The experimental learning-loop approach is presented and its basic elements are described in detail:

1. Adaptive femtosecond pulse shaping

2. Transient absorption spectroscopy

3. Evolutionary learning algorithm

A description of the electric field of a laser pulse is given together with an introduction to optical pulse shaping in frequency domain. The basics of transient absorption spectroscopy are given as well as how different processes and transitions express themselves in the measured transient spectra. A hypothetical example is used to explain how transient absorption spectroscopy can be used to extract molecular feedback for the learning loop. Evolutionary algorithms and related terms like 'learning curve', 'fitness function', 'mutation rate', etc. will become familiar. Furthermore, we will take a look beyond the closed-loop control. Some fundamental problems related to such a black-box experiment are discussed as well as means to overcome these hurdles. Finally, the idea of quantum control spectroscopy (QCS) will be introduced. 


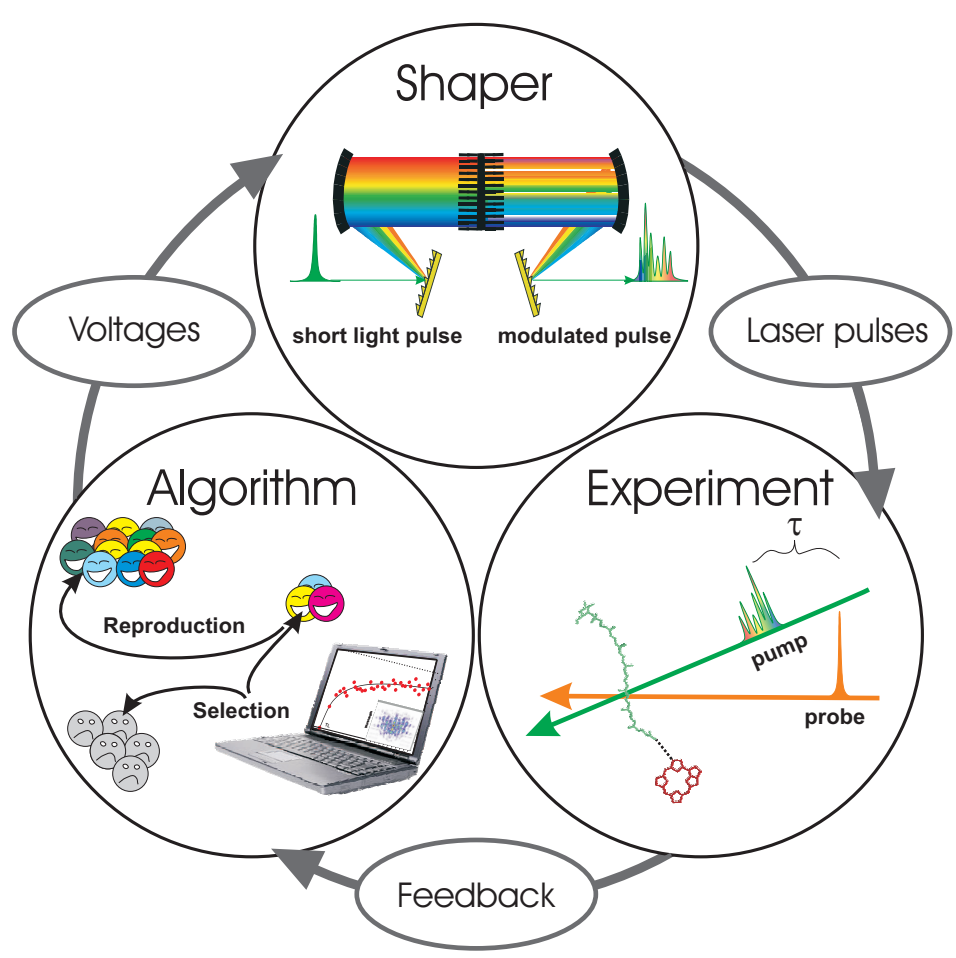

Figure 2.1: The basic elements of the learning-loop experiment. Voltages from the algorithm are transformed into pulse shapes in the pulse shaper and tested in an experiment to obtain molecular feedback. The algorithm creates new pulse shapes based on the molecular feedback.

The optimal control experiments utilise a closed-loop optimisation strategy [8, 21], whose basic elements are presented in Figure 2.1. The molecular systems studied here are complex and available theoretical quantum mechanical information is limited. For instance, the potential energy surfaces belonging to different electronic states are unknown, which makes it impossible to determine the driving optical field in advance. Therefore, we begin with so-called 'blind' optimisations, which means that we start without an initial guess, but rather a set of random phase patterns generated by the pulse shaper. The shaper modulates the dispersed spectrum of the input femtosecond laser pulse to create the shaped pulses. These shaped pulses are then tested on the sample and a feedback signal is derived based on the molecular response. The pulse shapes are then ranked according to how well they achieve the desired objective. A learning algorithm then uses the genetic information coded in the best pulse shapes to create new generation pulse shapes. Another iteration of the cycle may now begin, and the loop proceeds to search for pulse shapes that further increase the value of the feedback function, thus optimising the pulse shapes according to the set target objective. 


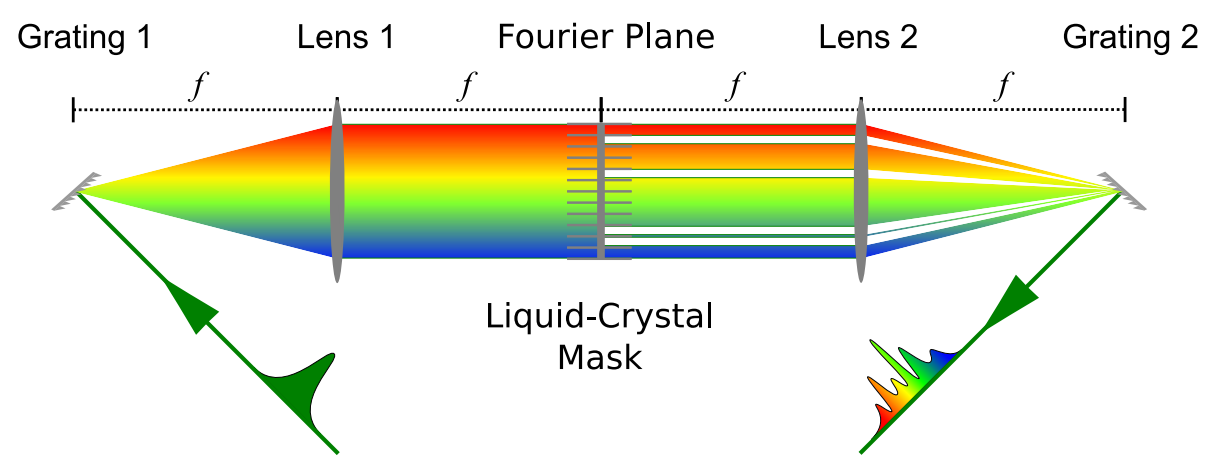

Figure 2.2: Schematic of the zero dispersion grating compressor layout of the 4- $f$ pulse shaper apparatus. An unmodulated pulse enters the shaper. Grating 1 and Lens 1 image the individual frequency components onto the Fourier plane, where the shaping is done by the SLM mask. Lens 2 and Grating 2 perform the inverse Fourier transform collecting the colours spatially and a shaped pulse emerges from the shaper.

\subsection{Pulse shaping}

A laser pulse is defined by its intensity and phase in either the time or the frequency domain

$$
\begin{aligned}
E(t) & =\sqrt{I(t)} e^{-i \varphi(t)} \\
\tilde{E}(\omega) & =\sqrt{S(\omega)} e^{-i \phi(\omega)},
\end{aligned}
$$

where $E(t)$ and $\tilde{E}(\omega)$ are the electric fields in time and frequency domain, respectively. $I(t)$ is the pulse intensity envelope in time and $S(\omega)$ the pulse spectrum, and $\omega$ is the carrier frequency. $\varphi(t)$ is the phase of the electric field in time and $\phi(\omega)$ the spectral phase.

Pulse shaping means manipulating the electric field according to

$$
E_{\text {out }}(t)=h(t) \otimes E_{\text {in }}(t),
$$

where $h(t)$ is the shaping function and $\otimes$ marks convolution. However, since the fastest electronic components operate with bandwidths that correspond to time responses in the picosecond regime, the femtosecond pulses are too fast to be manipulated directly in time domain and the shaping must be done in frequency domain:

$$
\tilde{E}_{\text {out }}(\omega)=H(\omega) \otimes \tilde{E}_{i n}(\omega),
$$

where $H(\omega)$ is now the shaping function in the frequency domain.

Figure 2.2 shows a schematic of a standard $4-f$ shaping arrangement for optical pulses. The incoming unshaped pulse is dispersed by a grating onto a focusing element that focuses the individual frequency components in the Fourier plane of the shaper. The phases and the amplitudes of the different frequency components are manipulated by a computer controlled liquid-crystal spatial light modulator (SLM) that is placed 


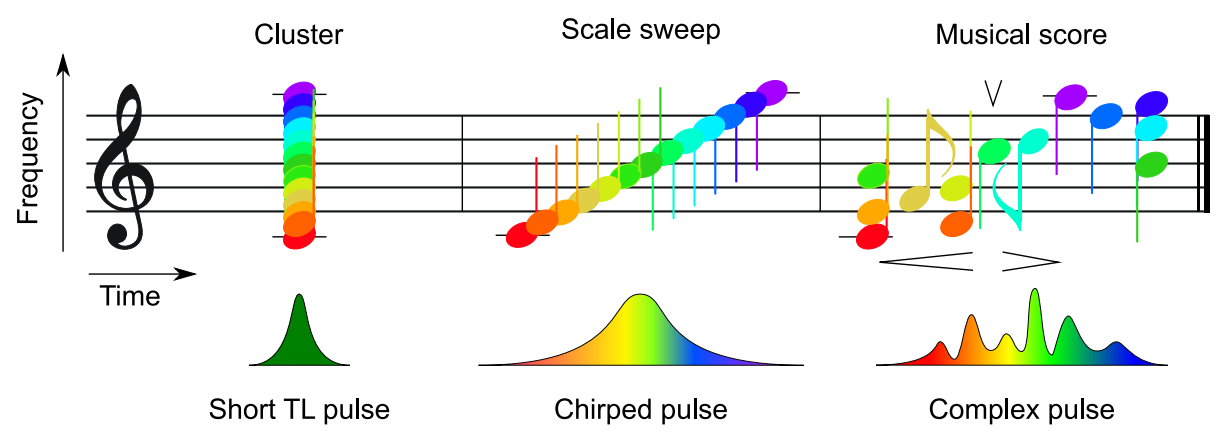

Figure 2.3: Analogy between the acoustic and the optical domain. The stave describes the time and the frequency information of music in the acoustic domain. Similarly, we can express a shaped pulse in time and frequency in the optical domain.

in the Fourier plane. After the SLM, the colours are collected back into one beam and the shaped output pulse exits the shaper. A more detailed description of a pulse shaper is given in Section 3.7.

Generally, any continuous function can be used to describe the spectral phase of a laser pulse. Sometimes, a polynomial form is practical

$$
\phi(\omega)=a_{0}+a_{1}\left(\omega-\omega_{0}\right)+a_{2}\left(\omega-\omega_{0}\right)^{2}+a_{3}\left(\omega-\omega_{0}\right)^{3}+\ldots,
$$

where $\omega_{0}$ is the centre frequency. $a_{0}$ is the absolute phase that describes the phase of the carrier frequency with respect to the field envelope. As our pulses are many optical cycles long we can use the slowly varying envelope approximation and neglect this contribution. The first order term produces a time delay, while the quadratic term creates a linearly increasing (decreasing) colour sweep in the time domain, which is more commonly known as the linear chirp. The following terms describe what is called higher order or nonlinear chirp.

Chirping of a bird is an useful analogy from the acoustic domain, in that it describes well how the frequency (colour) of a laser pulse changes as a function of time analogously to the frequency sweep of the pitch of a bird's singing. Taking this line of thought further, Figure 2.3 shows how the familiar acoustic domain can generally be used to illustrate the shaping of ultrafast laser pulses.

An ultrafast laser pulse is, in fact, a wavepacket that includes a spectrum of different frequencies. In the discrete form, the frequencies in the spectrum can be regarded as individual notes of a musical scale. In a transform-limited pulse where the $\phi(\omega)$ is constant, these frequencies all occur at the same time, which is like a 'cluster' of notes plucked at the same time. A linear chirp, corresponding to the quadratic term of $\phi(\omega)$ in Equation 2.5, is in the time domain analogous to a constant sweep across the scale. Finally, with a sophisticated pulse shaper we have control over the 'harmony' and 'melody' of the laser pulses and we can write highly complex optical scores on command. 


\subsection{Transient absorption spectroscopy}

An optimal control experiment imposes no restriction on how we 'communicate' with the molecular quantum system. In principle, the experiment can be anything from fluorescence measurement comparing the average emission between molecular species [22] to mass spectrometric detection of photofragmentation products [12], etc. We chose transient absorption spectroscopy to be the core experiment for extracting the molecular feedback for the algorithm. The choice relies mainly on three characteristics of the transient absorption technique. First, ultrafast laser pulses are the fastest things created by man and by using these pulses to excite and monitor we obtain a 'camera' with a shutter speed fast enough for assessing processes occurring on the molecular timescales. Secondly, from all ultrafast techniques available nowadays, transient absorption is perhaps the most versatile and flexible technique. It is capable of providing information on various different systems over a broad range of frequencies and on processes that occur on the ultrafast as well as on slower timescales. Finally, we want to utilise electronic resonances of molecules, which occur with transition frequencies from 200 to $800 \mathrm{~nm}$. Therefore, we need photons from the UV and visible region of the electromagnetic spectrum of light, which are readily available with a conventional pump-probe setup. This flexibility is of advantage in both the characterisation experiments and the control experiments. Transient absorption spectroscopy is introduced here conceptually, and the details of the setup are described in Chapter 3.

\subsubsection{Linear absorption}

Macroscopically, the exponential Lambert-Beer law describes linear absorption of light passing through a medium as a transmission change in the light. Accordingly, absorption or optical density (OD) is defined by

$$
\mathrm{A}=\mathrm{OD}=\epsilon C l=-\log \left(\frac{I(\lambda)}{I_{0}(\lambda)}\right),
$$

where $\epsilon$ is the molar absorption coefficient, $C$ is the concentration of the sample, and $l$ is the sample thickness. $I$ and $I_{0}$ are the transmitted intensities with and without the sample, respectively.

The molar absorption coefficient $\epsilon$ in Equation 2.6 links the observed absorption to a molecular quantity of absorption cross section $\sigma$, and further the quantum mechanical quantity of transition dipole moment $\mu$ [23]. $\mu$ is a measure of transition probability between molecular energy levels, and is linked to the molecular reference frame whereas $\sigma$ relates to the laboratory frame and averages all molecular orientations with respect to the polarisation of the excitation field. Further, the magnitude of the transition dipole moment belonging to a specific electronic transition depends on quantum mechanical quantities like the overlap integrals between the orbitals involved, selection rules, etc. [24, 25].

A traditional linear absorption spectrum gives a time-averaged picture of all the possible transitions between all resonant energy levels in the medium. Since, according to the Maxwell-Boltzmann distribution at room temperature [26], all molecules 


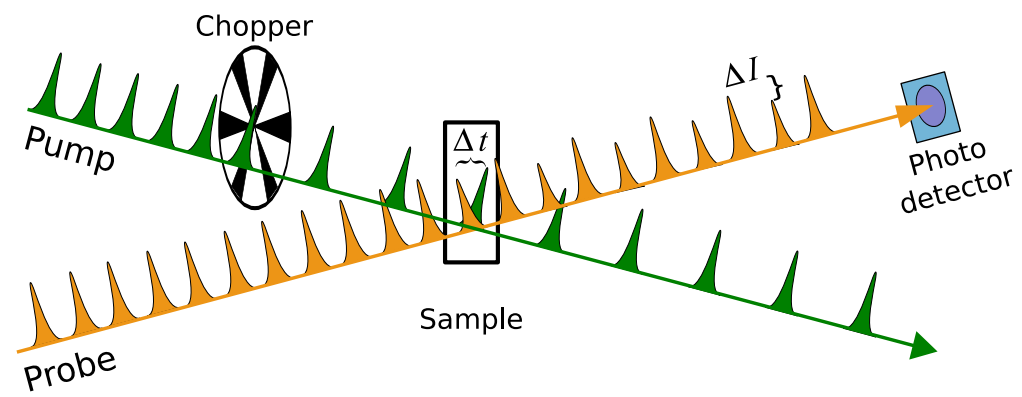

Figure 2.4: Transient absorption measurement. The pump pulses (green) are overlapped with the probe pulses (orange) at the sample. Every other pump pulse is blocked by the chopper. The pump induced difference in the transmitted intensity of the probe light $(\Delta I)$ is measured by a photodetector.

are at their electronic ground states, the lifetimes of transient energy levels or molecular species are generally short, and the intensities of the used electric fields are low, the linear absorption spectrum is almost completely dominated by the resonances belonging to transitions from the electronic ground-state up. To gain information on the transient molecular species and processes (e.g. populated energy levels, energy flow between them, and processes involving nuclear degrees of freedom like bond making and breaking, solvation, vibrational cooling, etc.), time-resolved spectroscopy must be used.

\subsubsection{Transient absorption}

The aforementioned molecular processes occur on timescales ranging from femtoseconds to nanoseconds. For example, an energy transfer process or the inertial part of dielectric solvation processes can take place in just few tens of femtoseconds whereas the lifetime of fluorescence emission is commonly some nanoseconds. Therefore, the time resolution has to be fast enough to measure the ultrafast molecular processes, while the temporal range has to be long enough to follow the course of these processes over longer periods of time.

Figure 2.4 shows the operational principle of the transient absorption spectroscopy. The ultrafast pump and probe beams are overlapped spatially at the sample and the pump induced transmission change in the probe light $(\Delta I)$ is measured. The transient absorption is given by

$$
\Delta \mathrm{A}=\Delta \mathrm{OD}=-\log \left(\frac{I(\lambda, \Delta t)^{\mathrm{ON}}}{I(\lambda)^{\mathrm{OFF}}}\right),
$$

where $I(\lambda, \Delta t)^{\mathrm{ON}}$ and $I(\lambda)^{\mathrm{OFF}}$ are the transmitted probe intensities when the pump pulse is ON or when pump is OFF, respectively. The difference between $I^{\mathrm{ON}}$ and $I^{\mathrm{OFF}}$ depends on the wavelengths of the pump and probe pulses, sample concentration, and the intensity of the pump pulse. Importantly, the $\Delta \mathrm{OD}$ is dependent on the time difference $\Delta t$ between the pump and probe pulses. The time scan $(\Delta t)$ is 
a

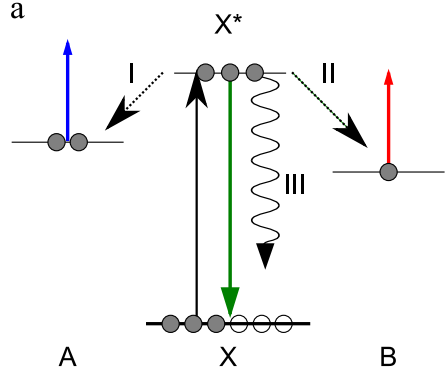

b

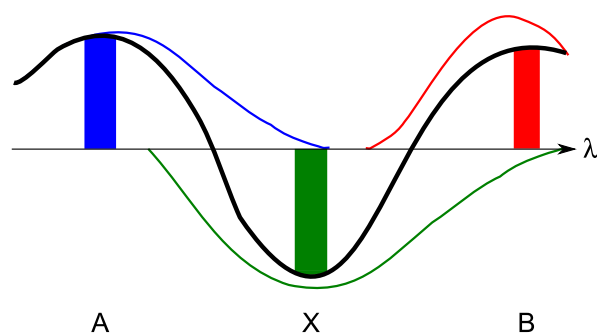

Figure 2.5: An energy-flow diagram and a transient absorption spectrum of a hypothetical system. Panel a: The pump pulse (black arrow, solid line) brings population to the excited state $\mathrm{X}^{*}$. Energy flows via two competing pathways I and II from $\mathrm{X}^{*}$ to A and B. An additional radiative process III is indicated by the wavy line. The coloured arrows show resonances belonging to transitions from A (blue) $\mathrm{X}^{*}$ (green), and B (red). Panel b: The species associated spectra belonging to bleach of $\mathrm{X}$ (green line), and induced absorption from A and B (blue and red lines). The corresponding transient spectrum at a time delay (black line) is a superposition of all contributing signals. The coloured bars represent the experimental feedback signals that can be used in the control experiments to monitor the populations in $\mathrm{X}, \mathrm{X}^{*}, \mathrm{~A}$, and $\mathrm{B}$.

achieved by delaying the probe pulse with respect to the pump pulse by increasing the beam path length with an optical delay line $(1 \mu \mathrm{m} \approx 3.34 \mathrm{fs})$. A transient spectrum simultaneously covering many transitions can be recorded by using spectrally broad probe pulses. In summary, high resolution time scanning combined with the extremely fast laser pulses and spectrally broadband probe pulses enables the desired time-resolved spectroscopy on the timescales of molecular processes.

\subsubsection{Transient spectrum and molecular feedback}

Panel a of Figure 2.5 shows a simplified energy-flow diagram of what could be a candidate molecular system for coherent control experiments. This hypothetical system has three competing pathways I, II and III from the initially excited state $\mathrm{X}^{*}$ taking population to $\mathrm{A}, \mathrm{B}$, and $\mathrm{X}$, respectively. $\mathrm{A}, \mathrm{B}, \mathrm{X}$, and $\mathrm{X}^{*}$ may symbolise electronic energy levels in the same or different molecules, or for instance, photoproducts of a photoisomerisation, or donor and acceptor species in an energy transfer process, etc. Similarly, I, II, and III can belong to a number of molecular processes. Generally, nonradiative processes of internal conversion and intersystem crossing as well as radiative processes of fluorescence and stimulated emission may take place. If a donor/acceptor system is present energy and/or charge transfer processes must also be considered.

After the excitation, $\mathrm{X}$ has less population in its ground state and a bleach/stimulated emission signal appears instantaneously (green arrow and bar). In this simplistic example, A and B have only induced absorption resonances to higher lying states (blue and red arrows and bars, respectively). The corresponding spectral bands appear in time with rate constants belonging to I and II. The magnitudes of the resonant signals belonging to transitions between different energy levels are governed by the corresponding populations and transition dipole moments, as explained above. 
Depending on the rate constants of the competing processes I, II and III and spectral signatures of $\mathrm{X}, \mathrm{X}^{*}, \mathrm{~A}$, and $\mathrm{B}$, a temporally and spectrally resolved feedback signal can be extracted for the control experiments as shown with the colour coded bars in Panel $b$. These signals are then further used to design a feedback function, for instance, to maximise the energy flow to channel I against channel II. In this case, the fitness function $f$ can simply be the ratio: $f=\mathrm{A} / \mathrm{B}$ at a chosen time delay. After evaluating the feedback function, we are ready to pass this information from the molecule to the algorithm that uses it in creating more favourable pulse shapes.

Even for a simple real molecule, the given example is an oversimplification. The participating energy-flow pathways are often complicated and the broad transient signals are overlapping each other. Furthermore, different processes related to solvation and nuclear degrees of freedom might play an important role in the dynamics of molecules. Therefore, real transient signals must be carefully studied in order to understand the details of the molecular dynamics and to resolve a possible reliable feedback signal for coherent control experiments.

\subsection{Evolutionary algorithms}

Evolutionary algorithms are stochastic search methods that mimic natural biological evolution. Evolutionary algorithms operate on a population of potential solutions applying the principle of survival of the fittest to produce better and better approximations to a solution. At each generation, a new set of approximations is created. First, by selecting individuals according to their level of fitness in the problem domain, i.e. their position on the fitness landscape. Secondly, by breeding them together using operators borrowed from natural genetics such as selection, recombination, mutation. The optimisation process leads to the evolution of populations of individuals that are better suited to their environment than the individuals that they were created from, just as in natural adaptation.

Evolutionary algorithms can be divided into three main categories: evolutionary programming [27], genetic algorithms [28], and evolution strategies [29, 30]. Although each has a different approach, they are all inspired by the principles of natural evolution. A good introductory survey can be found in references $[31,32]$. In this thesis work, algorithms from the class of derandomised evolution strategies are applied.

Figure 2.6 shows the progress of an optimisation. At the beginning of the computation, a number of individuals are randomly initialised. We call the number the population size and the set of individuals a generation. A fitness function is then evaluated for these individuals, where fitness corresponds to how well the individual achieves the target objective. If the optimisation criteria are not met, the creation of a new generation starts. Evolutionary operations take place inside the loop: Individuals are selected according to their fitness. The selected parents are then recombined to produce offspring and all offspring will be mutated with a certain probability. In case elitism is in use, some parents may survive as the offspring are inserted into the population. The new generation has been now created and the fitness of the offspring can be evaluated. This cycle is then repeated until the optimisation criteria are reached. The learning curve describes the learning process and depicts the fitness values of the best individuals of each generation. Usually, in the experiments the criterion for dis- 


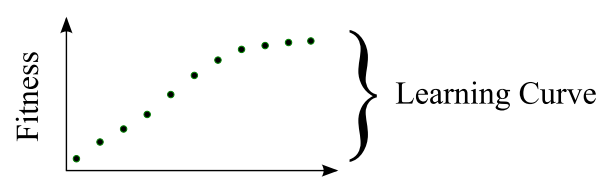

Generation \#

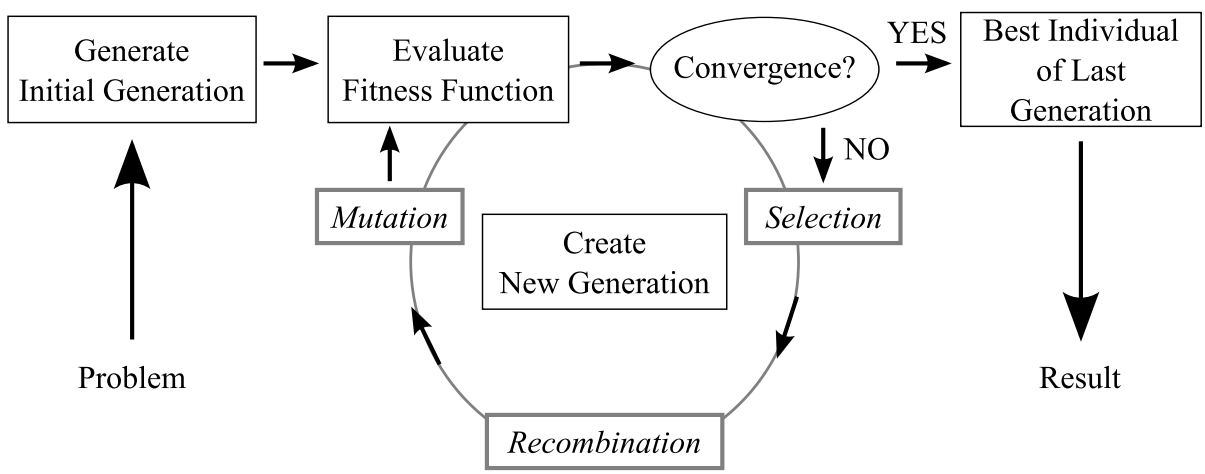

Figure 2.6: A flow diagram of an evolutionary algorithm. After the initial guess, the learning proceeds iteratively in a learning loop until the convergence to the chosen target objective has been reached. The best individual of the last generation represents the best approximation of a result to the original problem. The learning curve shows the fitness values of the best individuals of each generation.

continuing an optimisation is simply a good enough convergence, which is evaluated and decided by the user running the experiment.

Selection determines which individuals are chosen for mating (recombination) and how many offspring each selected individual produces. The first step is fitness assignment, which in this study was rank-based. In the next step, the actual selection is performed.

Recombination produces new individuals by combining the information contained in the parents.

Mutation is performed for every offspring after the recombination operation. Offspring variables are mutated by small perturbations with low probability. The extent of mutation is determined by the size of the mutation step and the probability by the mutation rate.

\subsubsection{Characteristics of evolutionary algorithms}

As evolutionary algorithms are the engine drivers of closed-loop optimisation experiments, it is worth discussing their characteristics a little further. Evolutionary algorithms differ substantially from more traditional deterministic search and optimisation methods: 
- Evolutionary algorithms search a population of points in parallel, not just a single point.

- Evolutionary algorithms use probabilistic transition rules, not deterministic ones.

- Evolutionary algorithms are generally straightforward to apply, because no restrictions for the definition of the target objective and the fitness function exist.

The complicated multidimensional fitness landscapes of coherent control experiments on molecular quantum systems are better explored by using evolutionary algorithms that sample the search space in parallel, rather than build a search path from a single point. The indeterministic nature of evolutionary algorithms is an advantage and a drawback: Since evolutionary algorithms do not repeatedly follow the same search path, a new experiment will scout new regions of the search space. This means that just by repeating the experiment new regions of the search space are explored, and for instance a local optimum can be avoided. However, it also makes repeating experiments impossible, since the search path once taken is unlikely to be followed again in a new experiment.

\subsubsection{Used evolution strategies}

For the experiments of this study, algorithms of the class of evolution strategies (ES) were applied. The most important feature of ES, distinguishing them from other evolutionary algorithms, is their ability to automatically adapt the distribution parameters of the underlying stochastic search. Since all evolutionary algorithms are stochastic search procedures by nature, they all rely on more or less advanced random search methods, usually hidden within the recombination and mutation operators. Yet most of these random search methods are build using some distribution function like a uniform or Gaussian distribution, which by themselves have defining parameters, for instance, standard deviations for Gaussians. The actual values of these parameters trivially influence the course of an optimisation: A Gaussian distribution with wide variances allows for big search steps, whereas a small variances allow for fine tuning. However, fixing parameters to one value for the entire optimisation may not be appropriate for efficient search, since coarsely identifying promising regions and converging to an optimal solution require different search distribution characteristics. This is commonly described as the contrast of exploration and exploitation of search methodologies. To overcome the weaknesses of fixed distribution stochastic search algorithms ES are capable of adapting their search distributions during the course of evolution along with the actual optimisation variables. This is commonly referred to as self-adaptation strategy. Different variants of ES most commonly differ in the self-adaptation mechanisms used. Well known approaches of self-adaptation are the $1 / 5$-th success rule of $1+1$-ES and stochastic self-adaptation originally suggested by Schwefel and Rechenberg [33] or, as used in this study, derandomised adaptation $[34,35]$.

The defining features of the derandomised ES used here, are related to the mutation operation. Generally, derandomised ES use a deterministic adaptation mechanism to derive new step size information from old step sizes and the magnitudes of successful mutation events. The core idea of derandomised step size adaptation is to compare 
the size of actual realisations of mutation events to the expected value of the originally proposed distribution. In the experiments we used two different adaptation schemes that are built on identical concepts but differ in the details of how the new step sizes are computed. The simple derandomised adaptation (DR2) basically adapts the $n$ variances of an $n$-dimensional Gaussian distribution [34], while the more advanced covariance matrix adaptation (CMA) uses the $n(n+1) / 2$ variances and covariances [35]. A detailed description of the used algorithms can be found in Chapter 4, where the performance of DR2 and CMA is evaluated against a real-life physical problem of optimal SHG in a nonlinear crystal, using simulations as well as laboratory experiments.

In general ES can be used both in an elitist and non-elitist fashion. The term 'elitist' refers to a detail of the selection mechanism that specifies which individuals can take part in the recombination process for creating new offspring individuals. If an evolutionary algorithm has an elitist type of selection old parent individuals are allowed to take part in creating new offspring as long as their fitness is better than any other individual's fitness. That means in an elitist strategy some individuals may survive forever. In a non-elitist strategy parent individuals are always discarded once new offspring has been created from them. Thus, in a non-elitist strategy each parent individual survives for exactly one generation. In common ES terms, the elitist strategy is a $(\mu+\lambda)$-ES and the non-elitist strategy is a $(\mu, \lambda)$-ES. Here $\mu$ describes the actual number of parents used and $\lambda$ describes the number of offspring derived from the $\mu$ parents. So the difference between the elitist $(\mu+\lambda)$-ES and the non-elitist $(\mu, \lambda)$-ES manifests in the set of individuals in generation $n$ that may become parents in generation $n+1$ : In a $(\mu+\operatorname{lambda})$-ES the best $\mu$ individuals of the $\mu+\lambda$ parent and offspring individuals of generation $n$ will become the new parent of generation $n+1$. In a $(\mu, \lambda)$-ES only the $\mu$ best individuals of the $\lambda$ offspring of generation $n$ may become parents of generation $n+1$. For this study only $(\mu, \lambda)$-ESs were used. This is for two reasons:

1. $(\mu, \lambda)$-ES have advantages for self adaptation of strategy parameters. In an elitist $(\mu+\lambda)$-ES individuals may survive forever that feature good optimisation variable values but inappropriate search distribution parameters which will inhibit optimisation progress and convergence.

2. For the desired application of the optimisation algorithm in the lab systematic decay of equipment and other measurement noise must be considered. In an elitist strategy individuals might survive that were accidentally conside rd too good because of measurement errors. Using an elitist $(\mu+\lambda)$-ES would require time consuming reevaluations of all $\mu$ parent individuals in each generation.

ES are commonly considered to mainly be driven by the mutation operator which is a contrast to e.g. genetic algorithms where the recombination operator is usually seen as the most important search mechanism. Due to the mutation-centric search of ES it is quite common to apply the extreme case of $(1, \lambda)$-ES, i.e. one parent search strategies that solely rely on mutation for search. In general this is a very efficient and usefull approach ${ }^{1}$.

\footnotetext{
${ }^{1}$ In fact the derandomised adaptation scheme originally as described in [34] was originally suggested as a $(1, \lambda)-E S$
} 

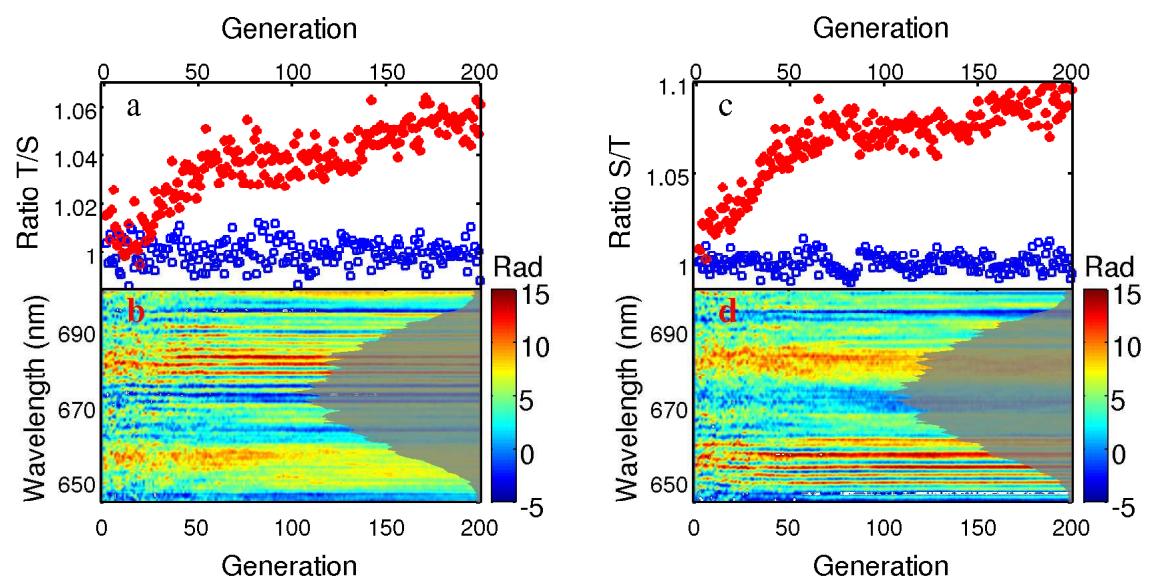

Figure 2.7: The learning curves and the phase surfaces from the optimal control experiments on the triplet yield of a photosensitiser zinc phthalocyanine. Panel a: Optimisation of the ratio Triplet/Singlet. The fitness of the best individual of each generation (red circles), and unshaped pulse (blue squares). Panel b: The phases of the best individual of each generation. Panel $c$ : Optimisation of the ratio Singlet/Triplet. The fitness of the best individual of each generation (red circles), and unshaped pulse (blue squares). Panel d: The phases of the best individual of each generation. The grey areas in Panels $\mathrm{b}$ and $\mathrm{d}$ show the pump spectrum.

In the presence of noise and measurement error in the evaluation function though using more than one parent is a promising approach to avoid getting trapped in false decisions. If no explicit noise control measures like multiple evaluations are implemented in the objective function there is the chance of overestimating the quality of a proposed solution. This may mislead evolution to some extend and therefore slow down both search and self adaptation. $(1, \lambda)$-ESs are unlikely to fail completely in this situation, since mutation will gradually adapt to this situation. Yet, using multiple parents with weighted recombination as suggested in [35] is may increase search efficiency. One aspect of the optimisation experiments performed in this study is finding good values for $\mu$ and $\lambda$ for the optimisation problems under study.

\subsubsection{Physical example}

Figure 2.7 shows an example of an evolutionary learning process in a coherent control experiment of a physical system. The optimisation of the phase of the laser pulse closes into the chosen molecular target objective, which can be seen in the increasing fitness values of the best individuals of each generation shown in Panels a and c (red circles). The molecule here is a photosensitiser zinc phthalocyanine and the target objective is to increase (decrease) the energy flow to the functional channel that brings the photosensitiser to the excited triplet state of the photosensitiser (see details in Chapter 8). Molecular feedback is resolved in a transient absorption experiment for the triplet (T) and singlet (S) signals with the fitness function defined to be $f=\mathrm{T} / \mathrm{S}$ 
in Panels a and $\mathrm{b}$, and $f=\mathrm{S} / \mathrm{T}$ in Panels $\mathrm{c}$ and $\mathrm{d}$. To verify that experimental conditions remained constant, the fitness of the unshaped pulse is evaluated before each generation (blue squares).

In 200 generations, some $\sim 6 \%$ and $\sim 10 \%$ increase is found for the ratios $\mathrm{T} / \mathrm{S}$ and $\mathrm{S} / \mathrm{T}$, respectively. The initial searching by the algorithm can clearly be seen in the Panels $b$ and $d$. The firts 50 generations show a lot of changes in the phase shape of the best individual. During this period the fitness landscape is being broadly searched and some favourable phase features are found. These features are then further refined as the algorithm approaches the best approximation to the solution. As expected, the optimal features differ between the optimal solutions that increase the two competing pathways.

One striking feature of the shown learning curves shown in Figure 2.7 is the noise. Even the fitness found with the unshaped pulse seems varies quite a lot. Obviously, this presents a challenge for the algorithm in the selection step, introducing uncertainty to the ranking. Chapter 4 presents a detailed study assessing this problem by comparing the performance and robustness of the two used algorithms using different initial settings.

\subsection{Challenges of black-box experiment}

However promising this kind of a learning-loop approach using evolutionary algorithms might seem, it also has a number of drawbacks and limitations. A detailed appreciation of the experiments and interpretation of the results presents a great challenge for the scientific community. Coherent control of chemistry is still a relatively new and emerging field and many of the fundamental questions remain yet to be resolved. In this section we raise some of these points related to the black-box nature of the closed-loop experiment.

Figure 2.8 shows the learning-loop experiment is actually a loop inside a superloop that the user actively controls. In this scheme, the learning-loop experiment (recall Figure 2.1) serves as a tool that is used to obtain optimal control. In other words, the user has a question and uses the black box to find the best answer to it. The superloop holds many fundamental issues concerning the different elements:

Question: Is the target objective well defined and physically feasible? Can we extract an unambiguous physical feedback signal? Is the answer included in the given search space? How can we avoid trivial answers?

The black box: Are the laser parameters adequate? Do the experimental conditions remain constant? Is the pulse shaper resolution sufficient? Is the $\mathrm{S} / \mathrm{N}$ ratio large enough? Is the algorithm smart enough?

Answer: Is the result the global optimum? Is the result robust? Does the result answer the question?

How we phrase the question is crucial for a successfull experiment. In order to choose criteria for the feedback signals and set the feedback function, the user must know the quantum system in good enough detail. The type and the size of the search 


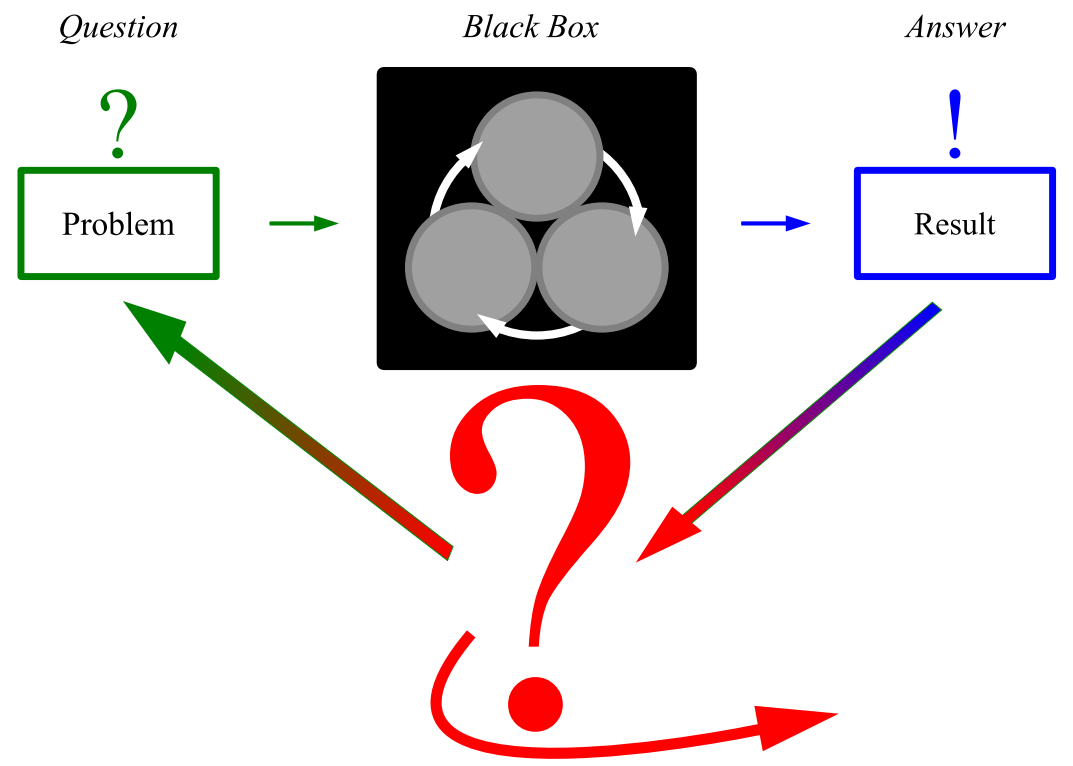

Figure 2.8: A flow diagram of an optimal control experiment that utilises the learning-loop black-box tool. The experimenter has a problem (green box) and formulates a corresponding question. After the decision to use the black-box, the experimenter initialises and starts up the learning-loop experiment. A result (blue box) emerges when the user decides to stop the experiment and the quality of the obtained answer can be evaluated. The red question mark symbolises the numerous questions and challenges arising from using such a black box tool. In the case of an insufficient or incomprehensible answer, the user may try to use the black box tool again, perhaps with better initialisation (red-green arrow) or perform other investigations (red arrow to outside the loop).

space must be carefully chosen to include the possible answer, but at the same time taking care that the number of parameters does not sky-rocket. The search space usually includes several trivial answers, which need to be taken into account in the initialisation step. Also, the initial parameter settings for the algorithm must be set right to ensure a learning process.

Operating the black box requires attention for an almost countless number of experimental variables and conditions. Also the type and the properties of the algorithm belong here. A great part of this thesis work was spend in improving the experimental techniques and details as well as developing the algorithm.

Due to the omnipresent experimental noise, the answer is always an approximation. The quality of the other parts of the experiment reflects directly on how good of an approximation we have obtained. Furthermore, the fitness landscape may suffer from ambiguities that are related to the physical problem itself and/or due to plain experimental limitations. Thus, the experimenter often rejoices at any result as it is often the only way forward and towards further learning by perhaps complementary techniques (see Section 2.5). 
It is the requirements for the question and the answer that ultimately define the control experiment. After the proposal of the learning-loop experiment in 1992 [8], a decade followed in the course of which a number of successful laboratory realisations of coherent control were reported. An overarching feature of all this work was the question: Can we control? During this period quantum systems with increasing complexity relented to the coherent control and yielded the answer: Yes, we can! However, it soon became clear that merely obtaining control brings very little new knowledge into the world and perhaps is not the most interesting goal for coherent control.

Today, as control has been succesfully imposed on various different quantum systems we are seeking answers to new questions. We now ask: Can we learn something new? What, and especially how, can we learn from the control? Thus, we are searching for answers beyond the learning-loop. The new goal is not only to obtain control but to understand the control, and to extract physical insights on the mechanisms behind control. This takes us to methodology outside the black box, and ultimately to quantum control spectroscopy (QCS).

\subsection{Outside the box}

We now discuss the methodology that takes us beyond the learning loop. This is done by presenting a brief overview of the existing methods that in one way or another can be categorised as complementary techniques to the learning loop experiment. Where some techniques take us back to the black box (Figure 2.8, red-green arrow), some take us to new experiments and/or analytical tools (Figure 2.8, red arrow). Nevertheless, the common feature for the first three techniques is that they are concerned in shedding light on the learning-loop results, whereas the open-loop and retracing methods seek answers also beyond the frame of optimal control.

\subsubsection{Pulse characterisation}

One result of an optimisation experiment is the best found pulse shape that in principle includes the solution worked out by the learning loop. I.e. we are searching an answer to an inverted problem: Can we extract microscopic information of the molecular system by analysis of the optimised laser field? Consequently, a 'standard' closed-loop coherent control research report includes pulse characterisation using techniques like auto- or cross-correlation, FROG, MIIPS, etc. [36, 37] as a common extra measurement. However, the mechanism is not always apparent or intuitively derivable just by analysing the pulse shape. As the systems become larger, the resulting pulse shapes become usually too complex to provide direct links to mechanism(s). Furthermore, it has been shown that even prominent pulse features may not correspond to key controls [38].

\subsubsection{Analysing search}

The resulting best pulse shape is just one outcome of the experiments. The pulse shapes and the corresponding fitness values along the learning curve can also be con- 
sidered as a result. In fact, they map out the path through the multi-dimensional search space that is followed by the algorithm. A course analysis can be used to extract physically meaningful parameters like subpulse distances in the course of the optimisation [39]. A mathematical tool, principle component analysis (PCA) can be used to analyse all pulse shapes in an optimisation to reveal principle control directions. PCA has been used to interpret control results on selective excitation of methanol vibrational modes [40]. In same fashion, dimension reduction by partial least squares regression analysis of the normalised covariance matrix of the whole data set has elucidated mechanisms behind two-photon fluorescence yield [41]. Dimension reduction by analysing the phases that were recorded along the learning curve in a free optimisation run was also used in the preliminary analysis of the control results in Chapter 7.

\subsubsection{Reducing search space}

One way to track down mechanistic insights is a stepwise experiment where the black box is reused. We start with an unrestricted 'blind' optimisation and then, after some analysis on the answer, return to the black box and conduct a second optimisation. The analysis step helps us to better phrase our question and prepare the next step. The performed analysis can be for instance the aforementioned pulse analysis or the PCA.

A common outcome of the analysis step is a reduced parameter space [10, 42]. Reducing the dimensionality by describing the search space with an analytical function may speed up the optimisation and, more importantly, reduce the complexity of the obtained result. In case the simplified space still contains the optimal solution more insights about the system become available as the complexity of the answer is reduced. This approach has been successfully applied in references [43, 44] and is used in Chapter 7. The importance of the basis of the search space has also been discussed relating to gas-phase fragmentation studies in references [45-47].

Another way to reduce the dimensionality of the search relates to the running of the black box. In this technique genetic pressure is applied on the spectral components using cost functions during the search. This pulse cleaning has been demonstrated with simulations [38] as well as experimentally [48, 49].

\subsubsection{Open-loop control}

Open-loop control experiments take the aforementioned basis reduction a step further. They are also a natural extension to the parametrisation of the search space. The resulting simplified space may turn out simple enough to be mapped entirely. Thus, in the experiments we map the chosen space by scanning a parameter. The nature and the dimensionality of these scans depends on the analytical function describing the reduced search space. Periodic, polynomial, and phase jump functions are typically used. The power of these scans has been demonstrated by manipulating wave-packet interferences in gas and liquid phase in references $[50,51]$ as well as in Chapters 7 and 8 . 


\subsection{Quantum control spectroscopy}

Coherent control is inherently sensitive to molecular dynamics. Therefore, by realising control we open a door to new insights to the interatomic forces that govern the function of molecular systems. However, as has been already implied, a successful closed-loop optimisation itself often tells us but little about the underlying physics: by running a learning-loop experiment it is perfectly possible to obtain control without actually learning anything new as pointed out in reference [10]. Therefore, we may argue that quantum control is only the first step on the path toward the more general goal of extracting new information. Fortunately, the above-described methodology provides several complementary smart-tools for the further investigations of the control. To use these complementary tools together with the learning loop to learn about the most intimate molecular-scale interactions is to perform quantum control spectroscopy (QCS) $[21,52,53]$. Being sensitive to the function and the goncerning microscopic-scale interactions hidden from conventional techniques, QSC offers several advantages and may ultimately take us closer to one of the penultimate goals of the field of coherent control: To find the coherent control 'rules of thumb', according which a given shaped pulse produces a predictable and universal response [21]. We conclude that the learning-approach and QCS are highly potential novel ways to look at the molecular world and the reviewed methodology bears numerous promises for fundamental research.

From a more prosaic point of view, the aforementioned new information and derived rules of thumb have a potential to serve countless applications [54]. Coherent control using learning loop provides us a handle to the function of molecular systems. A way to utilise this handle is to derive new design principles for chemical engineering. For instance, the new information can be on structrurally relevant motions that play an important role in the deactivation pathways of the molecular system [43]. Another practical aspect is to improve and refine existing analytical techniques, for instance to provide chemical selectivity for two-photon microscopy through tissue [55], or to realise coherent anti-Stokes Raman scattering microscopy with a single beam [56, 57]. Further, we can hypothesise ways of utilising control fields in direct applications of imaging, photomedical therapies, and quantum computing. Part II describes two applications relating to artificial light harvesting and cancer treatment photodynamic therapy. 



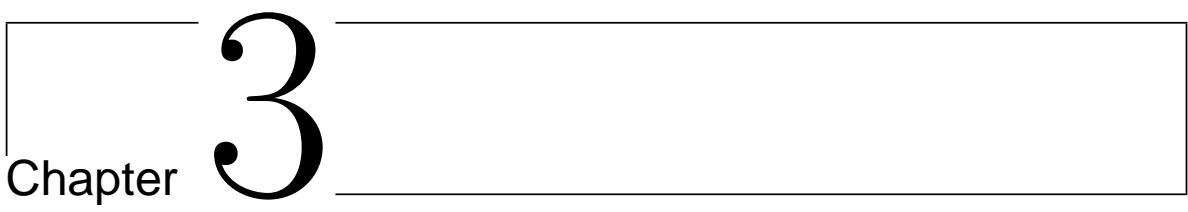

\section{Experimental setup}

This chapter introduces briefly the experimental tools used in this work. Designing and building the setup and various components within it, developing the software, and solving numerous fundamental practicalities formed a significant part of this thesis work. Therefore, we present an overview of these technical aspects of coherent control laboratory work. The laser system, the detection system, and other components are introduced. The key components - the noncollinear parametric amplifier and the femtosecond pulse shaper - are described in more detail. This experimental part will hopefully prove useful to experimental scientists aiming to perform similar work. To appreciate the following chapters, this technical chapter is not an essential read, and those who are more interested in the other aspects of the work may wish to skip these experimental details. 


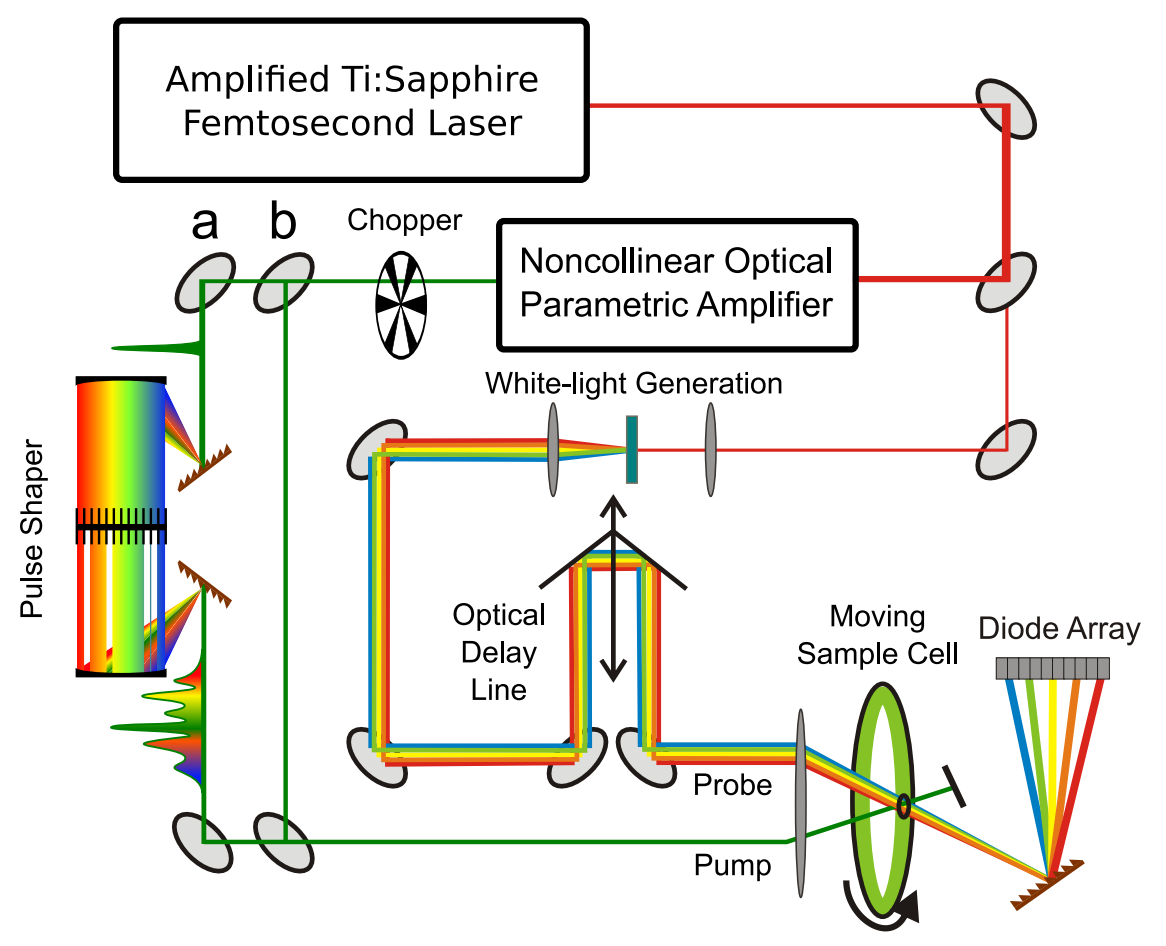

Figure 3.1: Schematic of the pump-probe setup.

\subsection{Transient absorption setup}

Ultrafast transient absorption spectroscopy is the core experiment of this thesis. ${ }^{1}$ It was the tool that provided both the molecular feedback in the coherent control experiments as well as the spectrally and temporally resolved data used to characterise the dynamics of the molecular systems under study.

A schematic of the setup in Figure 3.1 shows the main components of the transient absorption setup. The laser system provides the fundamental femtosecond laser pulses. The wavelength of the pump beam is tuned with a noncollinear optical parametric amplifier (NOPA) to the wavelength of a specific molecular absorption, which in this work belongs to a transition between two electronic states. A synchronised chopper blocks every other pulse to provide the $I^{\mathrm{ON}}$ and $I^{\mathrm{OFF}}$ (see Section 2.2) for the $\Delta$-absorption measurement. The laser output, the output of the NOPA, or a whitelight continuum (WLC), can all be used as the probe beam, depending on the spectral region of interest and the required time resolution. For coherent-control experiments the pump beam is coupled into the pulse shaper in path a, which in traditional pump-

\footnotetext{
${ }^{1}$ The pump-probe and coherent control experiments were complemented by other techniques like steadystate absorption and fluorescence spectroscopies. These are well known standard techniques, and are used here only for coarse characterisation before experiments, or as check-up techniques for sample degradation, and therefore are not described here in detail.
} 
probe measurements is bypassed via path $b$. An optical delay line is used to vary the time delay between the pump and the probe pulses. The pump and the probe pulses are focused and overlapped at the sample that is usually kept in a rotating cell or a flow cuvette. After the sample, the pump beam is blocked and the probe coupled into a house-build diode array detector via a spectrograph.

\subsection{Laser system}

The laser (CPA-2001; Clark-MXR Inc.) is actually three different lasers in a single box, and the creation of the fundamental laser beam proceeds in three consecutive steps. First, a seed beam is generated in the first floor of the laser housing by frequency doubling the output of a mode-locked diode-pumped erbium-doped fibre laser, lasing at $1548 \mathrm{~nm}$. The resulting $774 \mathrm{~nm}$ pulses at $\sim 34 \mathrm{MHz}$ repetition rate having a spectral bandwidth of $\sim 5 \mathrm{~nm}$ and a pulse energy of $\sim 40 \mathrm{pJ}$ are coupled into the second floor to be amplified. In the second floor, a grating pulse stretcher stretches the so-called injection pulses in time from $\sim 200$ fs to few ps by introducing linear chirp, and then passes the pulses to a chirped-pulse amplifier (CPA).

In the second step, pulses at $532 \mathrm{~nm}$ with a pulse duration of $\sim 300 \mathrm{~ns}$ are created in a Q-switched, mercury arc-lamp pumped, and frequency-doubled Nd:YAG laser providing approximately $7.1 \mathrm{~W}$ of average power at a repetition rate of $1004 \mathrm{~Hz}$.

In the third, and the final step the Ti:Sapphire crystal of the CPA is pumped with the high-power Nd:YAG beam in the cavity of the CPA. This is synchronised with a Pockel's cell pulse picker that picks one pulse every millisecond from the $34-\mathrm{MHz}$ pulse train of the injection laser for amplification. After some 20 cavity round trips the initial injection pulse is amplified $\sim 20 \times 10^{6}$ times in energy to $\sim 870 \mu \mathrm{J} /$ pulse. Output of the CPA is then coupled out with another Pockel's cell to a pulse compressor that removes the introduced extra chirp. Finally, the amplified pulses are coupled out from the box. All this is orchestrated by the control electronics and synchronised to the frequency of the mode-locked fibre laser.

Owing largely to the temperature controlled, nitrogen purged, all-in-one-box arrangement, the output characteristics of the laser system are usually stable and remain constant from day to day. The spectral width of the CPA output is $\sim 4.5 \mathrm{~nm}$ supporting approximately $200 \mathrm{fs}$ pulse lengths. Standard deviation of the pulse energy is $\sim 0.6 \%$, an important number for optimal control experiments that after the laser use nonlinear optical processes in creating the driving pump pulses as well as the probe pulses (see Appendix A).

\subsection{Noncollinear optical parametric amplifier}

For the purposes of this work, the characteristics of the fundamental laser pulses are insufficient. The need for improvement is twofold: in order to study different samples we need the capability to tune the wavelength of the excitation pulse to match the desired electronic resonances of the different molecular species under study. Secondly, the spectral bandwidth supports only $\sim 200 \mathrm{fs}$ pulses, which is not short enough for the experiments. Short pulses are required because the processes we characterise and control occur on molecular timescales and we seek better time resolution and more 
bandwidth to accurately resolve dynamical features of the molecules, as well as to allow for several pathways in controlling the molecules. Therefore, we make use of the noncollinear optical parametric amplifier $\left(\mathrm{NOPA}^{\mathrm{TM}}\right.$; Clark-MXR Inc.) [58-60].

In order to provide spectrally broad and tunable output pulses the NOPA ${ }^{\mathrm{TM}}$ utilises three different nonlinear optical processes: white-light continuum (WLC) generation, second-harmonic generation (SHG) (i.e. frequency doubling), and optical parametric amplification (OPA). These processes are introduced in Appendix A. Inside the NOPA $^{\mathrm{TM}}$ box, $4 \%$ of the $250 \mu \mathrm{J} /$ pulse input is focused into a sapphire plate to generate WLC that is used as the seed beam in the first OPA step. The bulk of the initial input energy is frequency doubled in a thin $\beta$-barium borate (BBO) crystal to create a $\sim 80-\mu \mathrm{J} /$ pulse pump beam at $387 \mathrm{~nm}$. The seed beam and $20 \%$ of the pump beam are overlapped in a 1-mm BBO crystal in space and time. The first OPA process produces spectrally broad signal pulses at a chosen centre wavelength. To increase the output yield these pulses are amplified further in a consecutive OPA booster stage using the signal from the first stage as a seed and the main part of the SHG beam as a pump.

The characteristics of the NOPA ${ }^{\mathrm{TM}}$ output vary with the chosen output wavelength and are always a subtle balance between output energy, width of the spectrum, beam profile, spacial frequency distribution, and pulse-to-pulse stability. As is shown in Figures 3.2, 3.5, and 3.4, optimising according to the requirements of different experiments normally gives an output with 7-25 $\mu \mathrm{J} /$ pulse energy, near-Gaussian beam profile, and a $20-50 \mathrm{~nm}$ spectral width. According to the uncertainty principle, for Fourier-transform limited pulses the minimum spectral width with a pulse duration of $\Delta t$ is

$$
\Delta \nu \Delta t=0.441
$$

If we assume a Gaussian shape for the spectral distribution, the shortest pulse supported by a pulse spectrum having a width of $\Delta \lambda$ in nm, can be calculated from

$$
\Delta t=\frac{0.441 \lambda^{2}}{c \Delta \lambda}
$$

where $\lambda$ is the centre wavelength, and $c$ the speed of light. Hence, a NOPA ${ }^{\mathrm{TM}}$ spectrum can theoretically support sub-10-fs pulses. In practice, pulse lengths are normally in $18-30$ fs range, giving a time-bandwidth product of $\sim 0.5$.

\subsection{Delay line}

To vary the time difference between pump and probe pulses an optical delay line is introduced to the probe arm of the setup. In fact, the setup makes use of two different delay lines in order to have a long time range and a good time accuracy. The shorter delay line (M-UTM150CC.1; Newport B.V.) has a $15 \mathrm{~cm}$ scanning range and a minimum step size of $0.1 \mu \mathrm{m}$, whereas the longer one (M-IMS600CC; Newport B.V.) has a $60 \mathrm{~cm}$ range and a minimum step size of $1 \mu \mathrm{m}$. The latter is used in a double-pass configuration enabling scans up to $\sim 7 \mathrm{~ns}$ at the same time with an accuracy of $0.668 \mathrm{fs}$ $(=0.1 \mu \mathrm{m})$. 


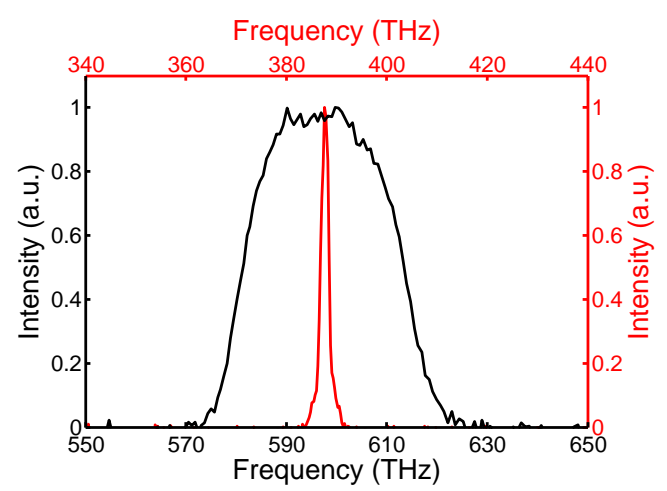

Figure 3.2: The CPA (red) and a typical NOPA $^{\mathrm{TM}}$ (black) output spectra. Spectra are overlapped using two $\mathrm{x}$-axes.

\subsection{Sample}

To avert any accumulation to long lived states and/or unwanted photochemistry, the samples needed to be moved during the experiments. Hence, the molecules were dissolved in appropriate solvents and placed in the rotating sample cell or a flow cuvette. The rotating cell holder was designed and built in house. The design used an air bearing, which provided an extremely stable rotation and ensured that the sample remained at the room temperature during the measurements. The cuvette rotating in the cell holder is a sandwich arrangement, comprising of a front window, spacer, and a back window. The front window is a thin microscope front glass to minimise the dispersion effects to the pump and probe beams. The back window is a $1 \mathrm{~mm}$ glass (BK7) window with a hole in the middle for injecting the liquid sample into the sample space. A ring of viton rubber having a thickness of $\sim 0.5 \mathrm{~mm}$ is used as a spacer between the front and the back windows. During the measurements, the speed of rotation was adjusted so that each pump pulse sees a fresh volume of sample.

The flow cell is a standard $1 \mathrm{~mm}$ thick quartz cuvette (QS-137-1-40; Hellma Benelux B.V.) connected with Teflon lined tubing to a gear pump. Again, the flow speed is adjusted to provide a fresh sample volume for each pump pulse.

\subsection{Detection system}

After the sample, the probe beam is coupled into the detection system consisting of a spectrograph (Acton-SP2150i; Princeton Instruments, Inc.) and a house-built diodearray detector (Diablo). The spectrograph has a focal length of $150 \mathrm{~mm}$ and can house different interchangeable gratings. Different gratings are needed for different applications. For pump-probe experiments, we used a grating blazed at $500 \mathrm{~nm}$ having a groove density of 300 grooves $/ \mathrm{mm}$. For the pulse shaper calibration and pulse characterisation we used gratings blazed at 500 and $300 \mathrm{~nm}$, having groove densities of 600 and 1500 grooves $/ \mathrm{mm}$, respectively. The spectrograph images the dispersed spectrum 


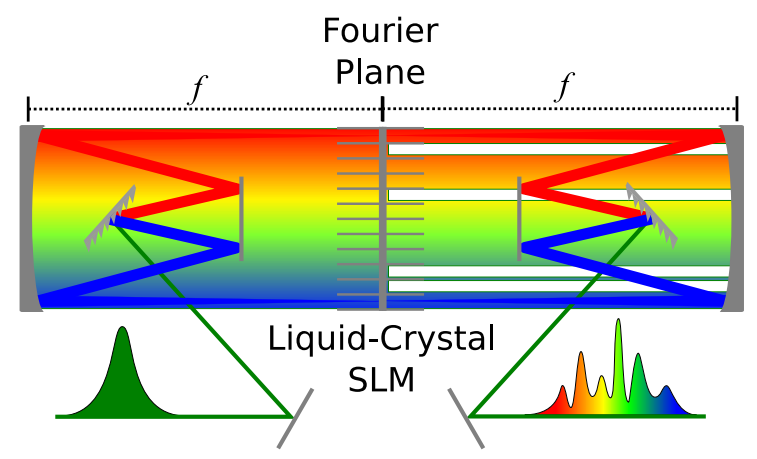

Figure 3.3: Schematic of the pulse shaper. An unshaped TL pulse enters the shaper. The individual frequency components are dispersed and imaged with a grating and a cylindrical mirror at the Fourier plane, where the liquid crystal mask is placed. After the mask, the colours are recombined in a symmetric setup and a modulated pulse exits shaper.

onto the diode array.

Diablo has two arrays having 256 pixels each. The pixel width is $50 \mu \mathrm{m}$ resulting in a $1 \mathrm{~nm} /$ pixel resolution and in pump probe experiments that use WLC for probing. The sensitivity of the diode array provides a detection range from $\sim 200 \mathrm{~nm}$ to $1100 \mathrm{~nm}$. The electronic design allows for $1 \mathrm{kHz}$ read out bandwidth, which makes it possible to have shot-to-shot statistics for individual pixels. This feature has a practical importance in day-to-day optimisation of the signal-to-noise ratio. The feature was also utilised in the evolutionary algorithm study presented in Chapter 4 . The shotto-shot signal statistics provided information on the experimental noise that was used to model the experiment accurately in the simulations.

\subsection{Femtosecond pulse shaper}

The pulse shaper comprises of a dual-stack spatial light modulator (SLM-1280-VN-R; Cambridge Research \& Instrumentation, Inc.), two 1800-grooves/mm gratings, folding mirrors, and cylindrical mirrors with $f=500 \mathrm{~mm}$ in a symmetric lay out shown in Figure 3.3.

The SLM placed in the Fourier plane has two liquid crystal masks with $640100 \mu \mathrm{m}$ wide pixels each. This leads to a theoretical resolution of $0.11 \mathrm{~nm} / \mathrm{pixel}$. The response time of the crystals is $\sim 35 \mathrm{~ms}$, and in the experiment a new phase pattern can be loaded every $\sim 50 \mathrm{~ms}$. The gap between the pixels is only $2 \mu \mathrm{m}$ and the SLM has a low attenuation of approximately $6 \%$. The main losses in the shaper come from the gratings and the throughput of the shaper is, depending on the wavelength, $\sim 50 \%$.

The liquid crystals in the two masks are oriented $\pm 45^{\circ}$ with respect to the direction $(\hat{x})$ of the polarisation of the electric field of the input laser pulses $\left(E_{i n}\right)$. The emerging 
field is given by [61]

$$
E_{\text {out }}=E_{\text {in }} e^{i \frac{\left(\Delta \phi_{1}+\Delta \phi_{2}\right)}{2}}\left[\hat{x} \cos \left(\frac{i\left(\Delta \phi_{1}-\Delta \phi_{2}\right)}{2}\right)+i \hat{y} \sin \left(\frac{i\left(\Delta \phi_{1}-\Delta \phi_{2}\right)}{2}\right)\right],
$$

where $\Delta \phi_{1,2}$ are the retardation of the frequency components when passing through the first and second layer. Using a polariser plate along $\hat{x}$ after the SLM enables phase and amplitude shaping. The transmission of a frequency component then becomes

$$
T \propto \cos ^{2}\left(\frac{\Delta \phi_{1}-\Delta \phi_{2}}{2}\right) .
$$

As we can see from equation 3.4, the difference between the two phases takes care of the rotation in the $(\mathrm{x}, \mathrm{y})$ plane and therefore of the amplitude modulation. The total output phase phase of a frequency component is

$$
\phi=\frac{1}{2}\left(\Delta \phi_{1}+\Delta \phi_{2}\right)
$$

Thus, the sum of the phases introduces a common phase, that is a delay. These two degrees of freedom make it possible to achieve at one time both amplitude and phase modulation of the spectrum of the input pulse.

\subsubsection{Calibration}

To realise such uncoupled phase and amplitude control over the spectral component of the input field the SLM must be carefully calibrated. Essentially, the calibration means finding a relation between the amount of phase retardation and the input voltage that is controlled by the computer. Only a careful calibration can ensure that the shaping introduces no unwanted amplitude shaping to the pump pulse. Therefore, we developed a robust calibration method, where each SLM pixel is calibrated.

We map the 640 SLM pixels to the 256 pixels of the spectrograph (see Section 3.6) and work on each separate color in the bandwidth of the input field by scanning the voltage of the whole mask at the same time. The resolution on Diablo is good enough to resolve the SLM pixels within the optical resolution of the shaper setup. In practice, a small 'smoothing' is present, which however does not reduce the quality of the calibration significantly.

In a calibration scan, one mask is fixed to the maximum voltage value and the voltage of the other mask is scanned over the necessary range. The spectrograph reads the amplitude variations in the output spectrum. After obtaining the $\cos ^{2}()$-shaped curve for each pixel, we extract the monotonic curve for each spectrograph pixel by an automated data processing, which also fits the resulting curves with a polynomial function to get a smooth calibration curve for each pixel. The obtained polynomial parameters are then interpolated to get the calibration curves for each SLM pixel. The quality of the calibration is verified by observing the leakage power when the amplitude shaping is set to zero over the whole SLM. Values less than $1 \%$ leakage at the worst wavelength are routinely recorded.

Following the calibration routine, the phase distortions in the input beam and the phase anomalies introduced by the shaper setup are compensated. The compensating 


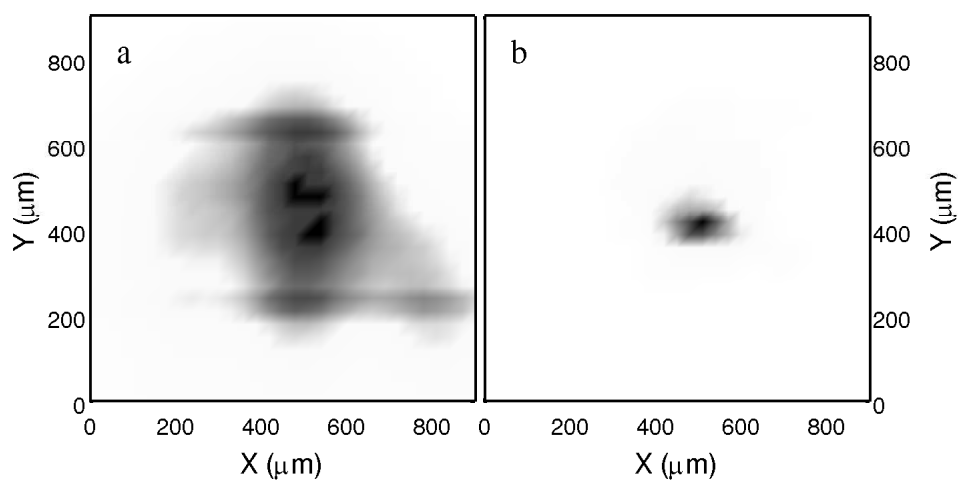

Figure 3.4: Beam profiles at the sample position. Panel a: The pump beam profile. Panel b: the probe beam profile. The 'stripes' in Panel a are due to a read-out error in the untriggered CCD imaging that interferes with the $1 \mathrm{kHz}$ laser frequency.

phase pattern is found by optimising SHG efficiency using a $10 \mu \mathrm{m}$ BBO crystal (see Chapter 4 and Appendix A).

\subsection{Pulse characterisation}

Naturally, the pulses from the laser, NOPA ${ }^{\mathrm{TM}}$ and the shaper need to be characterised. A fibre spectrometer provides (AvaSpec-2048; Avantes B.V.) a spectrum measurement at any position on the optical table. For intensity measurements a power meter (3sigma; Laser2000 Benelux CV) with various sensors (PM10, S10, and J5-09B) are used. Especially useful is the pyro detector (J5-09B) capable of sampling at $1 \mathrm{kHz}$ repetition rate providing statistic on the pulse energy.

The beam profile, the pump and the probe beam sizes as well as the spacial overlap between them at the sample position are determined by coupling the beams onto a CCD surface of a beam-profiler camera (Beam Profiler System; MS Macrosystem). This information is used to verify that the beams are optimally overlapped, and that the probe spot size is small with respect to the pump beam spot size, and thus sees a uniform pump intensity. Further, the pump beam profile allows accurate calculations of pump photon fluxes. An example of the pump and the probe beam profiles is shown in Figure 3.4.

Pulse lengths are measured with an autocorrelator $\left(\mathrm{NOPA}^{\mathrm{TM}}-\mathrm{Pal}\right.$; Clark-MXR, Inc.) using a split-mirror arrangement to provide two replicas of the original pulse that are then crossed in a BBO crystal to create the autocorrelation signal. Using a scanning stage with a piezo motor, the autocorrelator can make a real-time measurement of the autocorrelation function

$$
A C(\tau)=\int_{-\infty}^{\infty} I_{2 \omega}(t, \tau) d t=\int_{-\infty}^{\infty} I_{\omega}(t) I_{\omega}(t-\tau) d t
$$

where $I_{\omega}$ is the intensity of the input field and $I_{2 \omega}$ the intensity of the SHG autocor- 


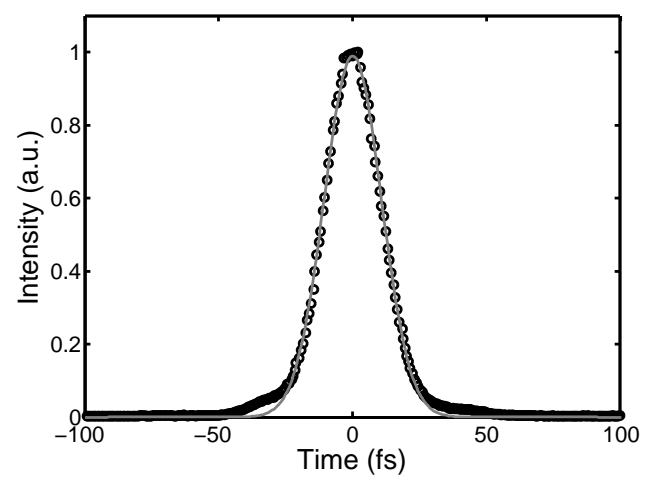

Figure 3.5: Typical autocorrelation of the $\mathrm{NOPA}^{\mathrm{TM}}$ output. The measured trace (circles) is fitted (grey line) with a Gaussian having a fwhm of $25.8 \mathrm{fs}$, which corresponds to a $\sim 18 \mathrm{fs}$ pulse length.

relation signal. An example of a typical autocorrelation of a 18 fs NOPA output is shown in Figure 3.5.

The shaped pulses are characterised more thoroughly using a frequency-resolved cross-correlation measurement (X-FROG)

$$
F(\tau)=\int_{-\infty}^{\infty} I_{\omega}^{\text {Shaped }}(\lambda, t) I_{\omega}^{\mathrm{TL}}(\lambda, t-\tau) d t,
$$

where $I_{\omega}^{\text {Shaped }}$ is the intensity of the shaped pulse, and $I_{\omega}^{\mathrm{TL}}$ the intensity of the transform limited gating pulse. For X-FROG measurements the sample is replaced with a $10 \mu \mathrm{m} \mathrm{BBO}$ crystal, and the cross correlation signal is resolved spectrally with the detection system. Figure 3.6 shows examples of experimental and simulated X-FROG traces. 

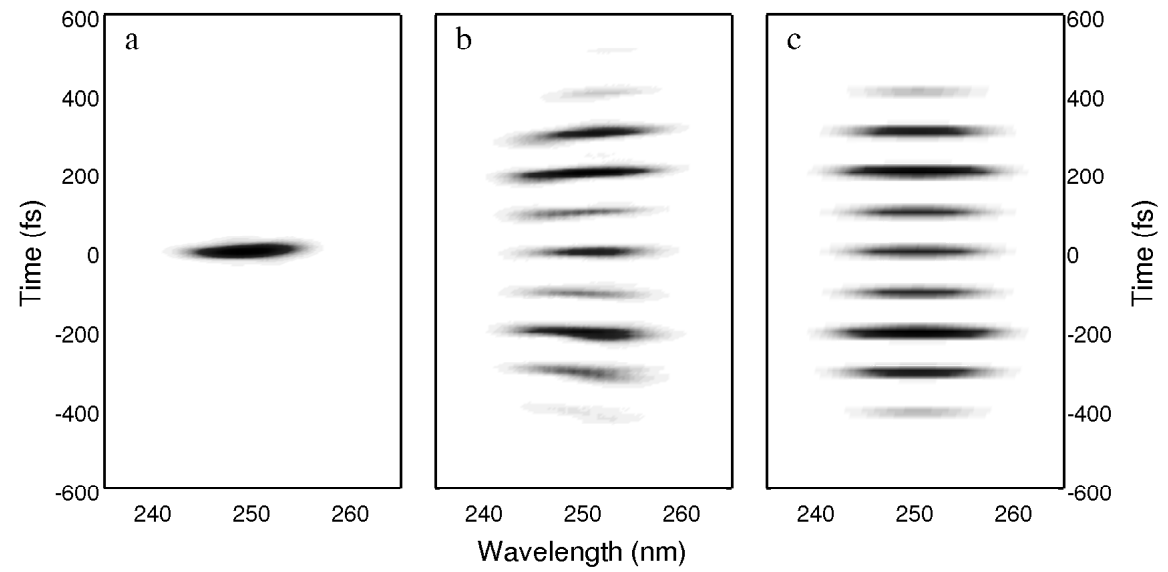

Figure 3.6: Examples of $X-F R O G$ traces of the shaper output. Panel a: SHG-optimised pulse. Panel b: An example pulse train. Panel c: X-FROG simulation of the pulse train. 


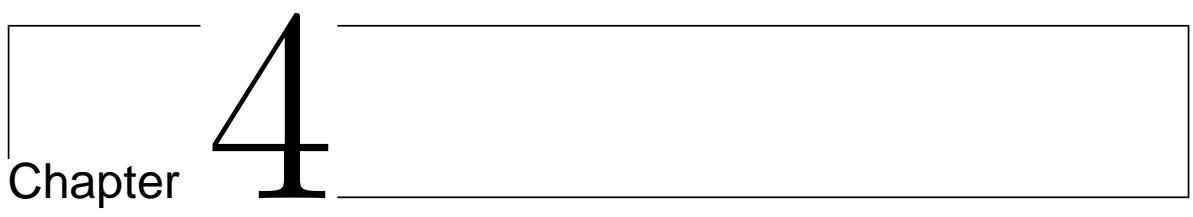

\section{Evolution strategies for laser pulse compression}

This study describes first steps taken to bring evolutionary optimisation technology from computer simulations to real world experimentation in physics laboratories. The approach taken considers a well understood laser pulse compression problem accessible both to simulation and laboratory experimentation as a test function for variants of evolution strategies. The main focus lies on coping with the unavoidable noise present in laboratory experimentation. Results from simulations are compared to previous studies and to laboratory experiments. 


\subsection{Introduction}

The use of evolutionary algorithms (EAs) is already widespread in several branches of science and engineering, whenever a process or product design involving highly nonlinear and complex operating conditions needs to be optimised at a pre-production stage of modelling or planning. In some cases, however, the system to be optimised cannot be adequately modelled or it needs to adapt to some (slowly) changing external parameters that cannot be predicted at a design stage. In these cases, when feedback is measured directly from the physical system, fitness evaluations will always be polluted by noise. The effect of this noise on the performance of the EAs needs to be minimised as much as possible. Generally speaking, one expects different EAs to respond differently to noise, and even the same EA will display a different degree of robustness to noise depending on the values of certain initial settings. In the present paper we report for the first time a study on the effect of real world noise on two commonly applied variants of EAs, namely evolution strategies (ES) using the CMA and the DR2 selfadaptation scheme.

Taking advantage of a physical experiment of laser pulse compression through optimisation (maximisation) of the intensity of second harmonic generation (a well understood process and an experimental set-up where noise can be monitored and controlled), we have run theoretical and experimental comparisons of CMA and DR2 as well as among different parameter settings of both algorithms.

Similar studies based on simulations of pulse shaping experiments have been performed in $[62,63]$. However, in contrast to the work presented here, the earlier simulations did not consider noise. Comparing results can reveal in how far algorithm performance changes due to the addition of noise. Additionally, in this study results of simulations are verified in the laboratory by physical experimentation.

\subsection{Second harmonic generation as fitness function}

The goal of our optimisations is to make the pulses coming out of our laser as short as theoretically possible. In order to do that we send the laser pulses through two devices that, at this point, we will consider as black boxes. First, a control box (pulse shaper) acts on the pulses to lengthen or shorten them depending on some optimisation parameters [61]. Second, a monitoring box (non-linear crystal) produces light at double the frequency of the input pulses by a process called second harmonic generation (SHG) [64]. As we will see in more detail later, the stronger the intensity of the SHG pulses, the shorter the initial laser pulses will be. This allows us to use the intensity of the SHG pulses as the fitness function for our EAs. Next we will go into a little more detail about the system, the way to achieve control over the input pulses and the creation of second harmonic light. 


\subsection{Introduction to optical second harmonic generation}

\subsubsection{Conceptual approach}

The effect of SHG of a laser beam through a crystal can be explained quite simply once some basic terminology is introduced. Whenever a pulsed laser is considered one refers to its shape and duration in time domain to characterise it. Imagining a plane being transversed by the laser pulse and recording over time the intensity of the light going through such a plane, the resulting plot of intensity vs. time is what is called the shape of the pulse in time domain. Now, to explain the concept of SHG, we will start from the idea that a pulse of light can be described as a superposition of sinusoidal functions with different frequencies and phases (Fourier description or description in frequency domain). The number of frequencies (i.e. colours) that are required to describe the pulse depends on its duration and on the complexity of its shape. The set of necessary frequencies is called the bandwidth of the pulse. The plot of the amplitude of these sinusoidals vs. their frequency is defined as the spectrum of the pulse, while the dependence of the phase term inside each sinusoidal on its associated frequency is the phase profile of the pulse. The spectrum tells us how much weight each sinusoidal has in describing the pulse, while the phase profile has to do with the relative delay of each frequency component with respect to a fixed reference (usually the frequency with the highest amplitude).

For our purpose a so called SHG crystal can be regarded as a black box in which all frequency components within the pulse spectrum are combined to give new sinusoidal components characterised by a frequency that is the sum of the input frequencies. When combining all these frequencies, the phase in each sinusoidal function plays a crucial role in determining the intensity of the SHG light in output.

\subsubsection{Mathematical approach}

Defining the pulse shape in time domain as $E(t)$ and its corresponding spectrum as $\tilde{E}(\omega)$, the SHG pulse in output from the crystal is given simply by:

$$
E_{S H G}(t)=E^{2}(t)
$$

In frequency domain, one can use the property of the Fourier transforms that relates a simple product of two functions in one domain to the convolution integral of the two functions separately transformed in the other domain:

$$
\tilde{E}_{S H G}(\omega)=\int_{-\infty}^{+\infty} \tilde{E}\left(\omega-\omega^{\prime}\right) \tilde{E}\left(\omega^{\prime}\right) d \omega^{\prime}
$$

If we change variable $\omega^{\prime}=\frac{\omega+\omega^{\prime \prime}}{2}$, we find that Equation 4.2 can be rewritten as:

$$
\tilde{E}_{S H G}(\omega)=\int_{-\infty}^{+\infty} \tilde{E}\left(\frac{\omega-\omega^{\prime \prime}}{2}\right) \tilde{E}\left(\frac{\omega+\omega^{\prime \prime}}{2}\right) d \omega^{\prime \prime}
$$


Equation 4.3 highlights better the fact that every frequency component $\omega$ in the $\tilde{E}_{S H G}$ spectrum is the result of the combination of a (infinite) number of frequency couples $\omega_{\text {left }}=\frac{\omega-\omega^{\prime \prime}}{2}$ and $\omega_{\text {right }}=\frac{\omega+\omega^{\prime \prime}}{2}$ whose center is located in $\omega$.

\subsection{Role of the phase}

The last step to understand our experiment of SHG optimisation consists in recognising the importance of the phase of the input pulse for the intensity of the SHG pulse in output. In our mathematical approach to SHG, the phase terms have been hidden until now in the functions $E(\omega)$.

In the next equations we pull the phases out.

$$
\begin{aligned}
\tilde{E}_{S H G}(\omega) & =\int_{-\infty}^{+\infty}\left|\tilde{E}\left(\frac{\omega-\omega^{\prime \prime}}{2}\right)\right| e^{i \phi\left(\frac{\omega-\omega^{\prime \prime}}{2}\right)}\left|\tilde{E}\left(\frac{\omega+\omega^{\prime \prime}}{2}\right)\right| e^{i \phi\left(\frac{\omega+\omega^{\prime \prime}}{2}\right)} d\left(4^{\prime} .4\right) \\
\phi(\omega) & =\phi_{0}(\omega)+\phi_{s h}(\omega)
\end{aligned}
$$

In Equation 4.5, now, the phase profile of the input pulse is explicitly written as the sum of two conceptually very different terms, the natural term $\phi_{0}(\omega)$ and a computercontrolled term $\phi_{s h}(\omega)$ introduced by means of our pulse shaper. The effect of the phase terms on the integral can be immediately understood if we regard the amplitude of the integrand as the length of a vector (length dependent on $\omega$ ) and the phase as the direction of such vector (also dependent on $\omega$ ). The best scenario that optimises the output of the integral is then clearly when all the vectors are pointing in the same direction. This case translates in all the vectors having the same phase (modulus $2 \pi$ ), which is tantamount to having a constant phase profile $\phi(\omega)$ in the input pulses. In an ideal pulse the term $\phi_{0}(\omega)$ should be constant as all frequencies should have the same phase when coming out of the laser. Unfortunately, in real life applications, as the pulse travels through optical tools like amplifiers, non-linear crystals for frequency tuning, simple lenses, beam splitters etc., it picks up a significant frequency-dependent phase profile that severely reduces the amount of SHG light coming out of our monitoring black box.

\subsection{Fitness and free parameters}

We define the fitness parameter for our evolutionary search as the total intensity of the SHG light generated in the crystal. In mathematical terms this would be:

$$
F=\int_{-\infty}^{+\infty}\left|\tilde{E}_{S H G}(\omega)\right|^{2} d \omega
$$

This function needs to be optimised by adding an adequate phase profile $\phi_{s h}(\omega)$ with our computer-controlled pulse shaper. In both our experiments and simulations, this additional phase function $\phi_{s h}(\omega)$ is described by 320 parameters free of varying within a $[0,2 \pi]$ range. The result of optimising a fitness function $F$, as defined 
above, is to make the total phase function $\phi(\omega)=\phi_{0}(\omega)+\phi_{s h}(\omega)$ a constant (modulus $2 \pi$ ), which, in return, yields the shortest possible pulse for the given bandwidth (transform-limited (TL) pulse).

\subsection{Properties of fitness function, search landscape, noise}

In this special case it is easy to prove that, modulus $2 \pi$, there is only one optimal solution with no local solutions. This lack of false directions or secondary solutions would seem to make the problem easy to solve. However, as we will show both with simulations and laboratory data, the most commonly used evolutionary algorithms do not always converge to the optimal, constant phase, solution. This behaviour is expected in the presence of noise in the fitness function $F$. In the following sections we will show a comparison between CMA and DR2 algorithms and we will try to find their best settings to handle a (realistic) noise level. After estimating the noise level of the fitness function $F$ in the laboratory, we modelled the SHG process based on Equations 4.3 and 4.6 and we introduced a random noise on top of the $F$ function according to Equation 4.7 and 4.8. Due to software requirements the original problem had to be turned from a maximisation problem to a minimisation problem. Equation 4.8 also expresses the fact that $F^{-1}$ was used as a fitness function ${ }^{1}$.

$$
\begin{aligned}
n_{c} & \sim \frac{5}{29} \cdot \mathcal{U}(0,1), \quad n_{v} \sim \frac{0.05}{29} \cdot \mathcal{U}(0,1) \\
F_{\text {opt }} & =F^{-1} \cdot\left(1+n_{c}\right)+n_{v}
\end{aligned}
$$

where $\mathcal{U}(0,1)$ denotes uniformally distributed random numbers from the interval $(0,1)$.

\subsection{Evolution strategies}

For this study two algorithms from the class of derandomised evolution strategies (ES) were applied. The defining feature of derandomised evolution strategies is a deterministic adaptation mechanism that derives new step size information from old step sizes and the magnitudes of successful mutation events.

The two variants applied here differ mainly in the distribution information that is adapted and used for creation of new offspring individuals: The simple derandomised adaptation (DR2) as suggested in [34] basically adapts the $n$ variances of an $n$-dimensional Gaussian distribution while the more advanced covariance matrix adaptation (CMA) as in [35] uses the $n(n+1) / 2$ variances and covariances. Though the details of the two adaptation schemes differ they are built on identical concepts. The core idea of derandomised step size adaptation is to compare the size of actual realisations of mutation events $(|z|)$ to the expected value of the originally proposed distribution $(E[|z|])$. Let $\sigma$ denote a parameter of the mutation distribution, and let $\sigma^{\prime}=A(\sigma, z, \theta)$ be the adapted parameter derived from the old $\sigma$, the successful mutation event $z$ and some internal parameters $\theta$. Then a derandomised adaptation function $A$ (i.e. here

\footnotetext{
${ }^{1}$ For future studies we would rather choose $-F$, but since $F$ is strictly positive using $F^{-1}$ is a feasible approach.
} 
either DR2 or CMA) basically implements a deterministic method that ensures the following conditions:

$$
\begin{aligned}
& E[|z|] \geq|z| \quad \longrightarrow \quad \sigma \geq \sigma^{\prime}=A(\sigma, z, \theta) \\
& E[|z|]<|z| \quad \longrightarrow \quad \sigma<\sigma^{\prime}=A(\sigma, z, \theta)
\end{aligned}
$$

That means that whenever a successful mutation is observed whose magnitude is smaller than what would be expected from the current step size the step size will be reduced and vice versa.

To stabilise the adaptation process both DR2 and CMA use the concept of cumulation path which means that the random variable $z$ used for adaptation is not directly the latest single successful mutation event but a weighted sum of successful mutations stretching over multiple generations. Therefore $z$ needs to be updated with successful mutation events $m$ by

$$
z^{\prime}=c m+(1-c) z
$$

where $c \in[0,1]$ is an algorithm specific constant ${ }^{2}$.

The implementations of DR2 and CMA used for this study also share the use of weighted recombination as introduced in [35]. Although the DR2 algorithm was originally suggested as a $(1, \lambda)$ strategy without recombination the adaptation scheme is sufficiently general to allow application of weighted recombination, i.e. new individuals are created by recombination

$$
x^{\prime}=x_{1: \mu} \cdot w
$$

where $x_{1: \mu}$ denotes the matrix of the $\mu$ best column vectors of design variables and $w$ is a weights vector with

$$
w_{i}=\frac{\log (\mu+1)-\log (i)}{\sum w_{i}}, \quad 1 \leq i \leq \mu .
$$

After recombination individuals are mutated by

$$
\begin{aligned}
m & \sim \mathcal{N}(0, \Sigma) \\
x^{\prime \prime} & =x^{\prime}+m
\end{aligned}
$$

where $m \sim \mathcal{N}(0, \Sigma)$ denotes sampling $m$ from a Gaussian distribution with expectation 0 and covariance matrix $\Sigma$.

\subsubsection{Handling box constraints}

As already mentioned in Section 4.6, optimal solutions to the SHG problem are unique modulus $2 \pi$. This introduces a certain difficulty for derandomised evolution strategies, since a successful mutation event might appear to be big on first sight, but reduce to a small change modulus $2 \pi$. In this situation the ES will falsely consider a too big mutation as a successful event. To avoid this kind of situation the Gaussian mutation

\footnotetext{
${ }^{2} c$ is usually assumed to depend on problem dimensionality.
} 
operator was slightly extended to ensure $0 \leq x_{i} \leq 2 \pi$ for all optimisation variables $x_{i}$.

The basic idea of a mutation is a small change to the original data, such that evolution proceeds as a series of minor changes rather than a big singular, dramatic change. This is one of the main reasons for using Gaussian distributions as mutation operators in evolution strategies [33]. Since Gaussian distributions are at least theoretically unbounded the usual mutation operators as defined in Equations 4.14 and 4.15 cannot assure upper or lower bounds on the outcoming, mutated optimisation variable $x$. Assume box constraints of the form $l b \leq x \leq u b$, where $l b$ and $u b$ denote lower and upper bounds on $x$, respectively. We propose a repair method working on top of the usual Gaussian mutation, where the following conditions should hold:

1. Whenever an infeasible Gaussian mutation event is detected, the new mutation operator should yield a mutation that is smaller or equal to the Gaussian mutation event. By doing so, we assume that the Gaussian mutation event always classifies as a small change, and ensuring that the new mutation event is even smaller and therefore qualifies as a mutation like small change.

2. Whenever an infeasible Gaussian mutation event is detected, the new mutation operator should create a mutation in the same direction, i.e. if the Gaussian mutation event increased $x$ then the new mutation event should also do that and vice versa. This is different from a simple resampling approach, where Gaussian mutations are performed repeatedly until a feasible value is generated. Resampling biases resulting values away from the interval bounds, which is undesirable.

To implement this we suggest to mutate Gaussian first, and whenever that yields infeasible values, to replace the Gaussian sampling by a uniform sampling in the interval given by the original $x$ and the respective bound violated by the Gaussian mutation event. E.g. if the Gaussian mutation violates $l b$ the mutation samples uniformly in the interval $[l b, x]$. Doing so of course assumes that $x$ is always feasible, but that should not be problematic by initialising feasibly. This approach is formalised in the following equations:

$$
\begin{aligned}
m_{0} & \sim \mathcal{N}(0,1) \\
x^{\prime} & =\left\{\begin{array}{lll}
x+s \cdot m_{0} & \text { if } & l b \leq x+s \cdot m_{0} \leq u b \\
x+\mathcal{U}(0,1) \cdot(x-l b) & \text { if } & x+s \cdot m_{0}<l b \\
x-\mathcal{U}(0,1) \cdot(u b-x) & \text { if } & x+s \cdot m_{0}>u b
\end{array}\right. \\
m & =\left(x^{\prime}-x\right) \cdot s^{-1}
\end{aligned}
$$

where $\mathcal{U}(0,1)$ denotes sampling uniformally from $[0,1)$. Equation 4.18 is required for path accumulation (Equation 4.11) during derandomised adaptation. For CMA and DR2 the repair mechanism simply pretends that the mutation event was originally created by Gaussian mutation, such that search distributions can be adapted appropriately. 


\subsubsection{Derandomised adaptation}

The derandomised adaptation approach (DR2) used in this study was first introduced in [34]. For an $n$-dimensional optimisation problem it uses $n+1$ strategy or step size parameters, where one $\left(\sigma_{0}\right)$ defines the global width of the Gaussian search distribution and the remaining ones $\left(\sigma_{i}, 1 \leq i \leq n\right)$ define the variances of $n$ independent Gaussian distributions used to mutate the corresponding $n$ optimisation variables. Equation 4.19 shows the working principal of the adaptation scheme using the accumulated path $z$ as defined in Equation 4.11.

$$
\sigma_{0}^{\prime}=\sigma_{0} \cdot \exp \left(c_{1}\left(\frac{|z|}{c_{2}}-E[|z|]\right)\right), \quad \sigma_{i}^{\prime}=\sigma_{i} \cdot \exp \left(c_{3}\left(\frac{\left|z_{i}\right|}{c_{4}}-E\left[\left|z_{i}\right|\right]\right)\right)
$$

where $E[|z|]$ and $E\left[\left|z_{i}\right|\right]$ denote approximations to the expected value of the length of the $z$ vector and the absolute value of its components, respectively, and $c_{1, \ldots, 4}$ are normalisation constants. For further details sees $[34,65]^{3}$.

\subsubsection{Covariance matrix adaptation}

The following equations give a bird's eye view of the main aspects of the covariance matrix adaptation (CMA) scheme. Further details, especially on the vastly simplified setting of the various normalisation constants, can be found in [35]. Consider $\Sigma$ the covariance matrix to be adapted and $B$ the corresponding matrix of eigenvectors and $D$ the corresponding eigenvalues, and let $\mathcal{N}(\mu, \Sigma)$ denote a Gaussian random variable with expectation $\mu$ and covariance matrix $\Sigma$. The eigenvectors and eigenvalues are used for rotation and scaling such that a vector of independently $\mathcal{N}(0,1)$ distributed Gaussian random variables can be turned into a vector of $\mathcal{N}(0, \Sigma)$ distributed Gaussian random variables and vice versa. Assume further that $m_{0,1}$ is the vector of $\mathcal{N}(0,1)$ distributed Gaussian random variables that happened to be a successful mutation ${ }^{4}$.

CMA first of all adapts a global scalar step size $\sigma_{0}$ that determines the overall width of the next search distribution. To do so CMA compares the length of the accumulated successful mutation information vector to its expected value $E\left[\left|z_{\sigma_{0}}^{\prime}\right|\right]$.

$$
z_{\sigma_{0}}^{\prime}=c_{1} z_{\sigma_{0}}+c_{2} B m_{0,1}, \quad \sigma_{0}^{\prime}=\sigma_{0} \exp \left(c_{3}\left(\frac{\left|z_{\sigma_{0}}^{\prime}\right|}{E\left[\left|z_{\sigma_{0}}^{\prime}\right|\right]}-1\right)\right)
$$

The actual covariance matrix is adapted after accumulation by adding a rank one matrix yield from multiplying the accumulated path information by itself.

$$
p_{\Sigma}^{\prime}=c_{4} p_{\Sigma}+c_{5} B D m_{0,1}, \quad \Sigma^{\prime}=c_{6} \Sigma+c_{7} p_{\Sigma}^{\prime} p_{\Sigma}^{\prime T}
$$

\subsection{Second harmonic generation: Simulations}

The simulation study considers the following parameters of the optimisation algorithms:

\footnotetext{
${ }^{3}$ In [34] the adaptation of the local step size $\sigma_{i}$ in Equation 4.19 has a slightly different form, yet in our experience both versions perform equally well.

${ }^{4}$ In accordance with Equations 4.14 and $4.15 m=B D m_{0,1}$ holds true.
} 


\section{Adaptation Mechanism}

The core method implementing self adaptiveness of the evolution strategy.

\section{Number of Parents}

The $\mu$ parameter in a $(\mu, \lambda)$ evolution strategy

\section{Number of Offspring}

The $\lambda$ parameter in a $(\mu, \lambda)$ evolution strategy

\section{Initial Step size}

The magnitude $\sigma_{0}$ of the initial step sizes, i.e. width of the search distribution.

These parameters are probably the most frequently used parameters to tune evolution strategies. Although by now there are useful suggestions available for automatically setting population sizes [35] these heuristics are not necessarily applicable for noisy functions. It has been observed before [66] that population sizes are highly influential for noisy fitness functions. From early on the numbers of parents and offspring have not been considered as independent parameters (see e.g. [33]), it has rather been assumed that the ratio of $\mu$ and $\lambda$ is a key parameter. Therefore, in this simulation study, combinations of a number of parents $\mu$ and the parent to offspring numbers ratio $\mu / \lambda$ were tested rather than combinations of $\mu$ and $\lambda$ directly. The following parameter values were combined for the simulation study:

$$
\mu \in\{1,5,10,20\}, \quad \frac{\mu}{\lambda} \in\left\{\frac{1}{2}, \frac{1}{4}, \frac{1}{10}\right\}, \quad \sigma_{0} \in\{0.2,0.1,0.01\}
$$

Table 4.1 summarises some of the results achieved with simulation runs. First of all the table contains the best parameter sets detected. As quality measure the median of the best fitness value recorded in 15 repetitions of the same optimisation run was used. Table 4.1 also shows the mean, minimum, maximum, and standard deviation of the 15 best fitness values associated with each parameter set. Additionally some of the worse results are listed for reference. Due to limited space not all results can be given. It is obvious from the table that with the exception of the last row there are no trials listed using the smallest initial step size of $\sigma_{0}=0.01$. It turned out that almost all optimisations using this small step size achieved only very bad results. This can be seen in Table 4.2, where performance metrics were aggregated over all results with identical initial step size. The aggregation averages over very differently performing strategies, as can be seen from the respective minimum, maximum, and standard deviation columns. Still there seems to be a dramatic difference in the mean and median values for $\sigma_{0} \in\{0.1,0.2\}$ on the one hand and $\sigma_{0}=0.01$ on the other hand.

Coming back to the results of Table 4.1 significant differences between the SHG problem with and without added noise can be seen. While in [63] the $(1,10)$-ES using DR2 adaptation outperformed a $(1,10)$-ES using CMA adaptation, which itself outperformed an $(8,17)$-ES using CMA, the addition of noise clearly discards the $(1,10)$-strategies, which finished with ranks 35-38. Interestingly though, within the set of $(1,10)$-strategies DR2 still performs better than CMA, so one might conclude that DR2 manages the basic characteristics of the SHG problem more successful than CMA, without noise changing this big picture for a $(1,10)-\mathrm{ES}$. 
From Table 4.1, no clear answer to the question of which adaptation scheme to prefer can be deduced. Although the table contains more well performing results based on CMA than on DR2, the second best performing strategy uses DR2. The fact that there are more rows filled with CMA results than with DR2 results basically means that CMA is less sensitive to its parameter settings, i.e. it is less critical to determine good population sizes for CMA than it is for DR2. In general, a certain improved reliability turns out to be an advantage of CMA that is also visible in the standard deviation column of Table 4.1. Apart from less sensitive parameter settings CMA's higher reliablity can also be seen in smaller variance of optimisation results. With the exception of the $(10,40)$-ES using DR2 all CMA results have considerably less spread than their DR2 counterparts.

\subsection{Second harmonic generation: Laboratory}

One of the main motivations for this study apart from studying the influence of laboratory noise on optimisation performance was to assess the transfer of knowledge gained from simulated optimisation runs to real world laboratory experimentation. This is largely because laboratory time is considerably more expensive and restricted than computation time. A single optimisation run in the laboratory takes up to 30 minutes, neglecting considerable time to set up the experiment correctly. Due to limited laboratory infrastructure optimisation runs cannot be parallelised which is most easily done with simulations. So in the light of limited experimentation time available for this study we decided to trade widespread parameter testing for statistical significance, and ran multiple repetitions of a limited number of paremeter settings in the laboratory. In order to not bias results by selecting only the best parameter settings for both CMA and DR2, the best, a mediocre, and a rather bad setting as found in the simulations were tested. In Table 4.1 these settings are marked with a $\star$.

Table 4.3 summarises the results achieved in the laboratory together with the respective simulation experiments. For technical reasons the numerical values for simulations and experiments in the table do not match exactly. To extract more meaningful information on the link between simulations and experiments, we separately normalised both type of results to their best value. Comparing these ratios in the last two columns of Table 4.3 we can see how the results of simulations and experiments are strikingly similar.

In contrast to the simulation results, though, CMA yields better results than DR2, except when used with the much too small step size of $\sigma_{0}=0.01$. The issue of too small initial step size is likely to be more problematic in the laboratory than it was for simulations, since there are potentially additional sources of noise acting on the inputs that were not covered during simulations. Too small initial step sizes may easily lead to variable changes on the same scale as the noise, which makes optimisation practically impossible.

\subsection{Conclusions and outlook}

In this study we compared two different adaptation schemes of evolution strategies, namely DR2 and CMA, together with variations of their parameter settings in a proto- 
type laboratory situation. We found significant differences between simulations with and without added noise. Nonetheless, the results from the noisy simulations transferred nicely to the laboratory, as verified by a number of experiments. Although both variants of the evolution strategy in principle produced good results both in simulations and in the laboratory, CMA turned out to be the more reliable algorithm. Since reliability is of fundamental importance in the laboratory, CMA is considered the method of choice for future experimentation.

The results of this study suggest that Second Harmonic Generation can be used as a prototype application for online control of laboratory experiments by evolutionary algorithms. The obvious next steps are improvement of algorithm performance for the targeted noisy fitness functions on the one hand, using SHG as a primary test function. On the other hand, more challenging applications can be addressed with the knowledge gained by the SHG experiments. Especially laboratory optimisation experiments for which today no feasible computer simulations are available, may be tackled using the methods and insights available from continuing improvement of the algorithms. 
Table 4.1: Performance of different parameter settings after 5000 evaluations showing median (Median), mean (Mean), minimum (Min), maximum (Max), and standard deviation (Std) of the best fitness values of 15 repeated optimisation runs. The Rk column indicates the position of the row if ordered by the respective value. Rows marked with $\star$ were tested in the laboratory.

\begin{tabular}{|c|c|c|c|c|c|c|c|c|c|c|c|c|c|c|}
\hline & Alg & $\mu$ & $\lambda$ & $\sigma_{0}$ & Median & $\mathbf{R k}$ & Min & $\mathbf{R k}$ & Max & $\mathbf{R k}$ & Mean & $\mathbf{R k}$ & Std & $\mathbf{R k}$ \\
\hline 夫 & CMA & 20 & 40 & 0.10 & 2.471 & 1 & 2.207 & 2 & 3.117 & 2 & 2.525 & 1 & 0.229 & 4 \\
\hline \multirow[t]{7}{*}{$\star$} & DR2 & 20 & 80 & 0.20 & 2.703 & 2 & 2.073 & 1 & 3.932 & 9 & 2.834 & 3 & 0.532 & 13 \\
\hline & CMA & 20 & 40 & 0.20 & 2.716 & 3 & 2.441 & 4 & 3.114 & 1 & 2.747 & 2 & 0.210 & 3 \\
\hline & CMA & 10 & 40 & 0.10 & 2.876 & 4 & 2.515 & 6 & 3.928 & 8 & 3.024 & 5 & 0.462 & 11 \\
\hline & CMA & 10 & 20 & 0.10 & 2.937 & 5 & 2.453 & 5 & 3.291 & 3 & 2.981 & 4 & 0.237 & 5 \\
\hline & DR2 & 10 & 100 & 0.20 & 2.938 & 6 & 2.432 & 3 & 6.258 & 22 & 3.248 & 8 & 0.988 & 27 \\
\hline & CMA & 10 & 20 & 0.20 & 3.018 & 7 & 2.635 & 7 & 3.759 & 7 & 3.047 & 6 & 0.355 & 8 \\
\hline & DR2 & 10 & 40 & 0.20 & 3.125 & 8 & 2.740 & 8 & 3.613 & 4 & 3.192 & 7 & 0.271 & 7 \\
\hline \multirow{4}{*}{\multicolumn{2}{|c|}{$\begin{array}{c}\text { CMA } \\
\text { CMA } \\
\text { CMA } \\
\text { DR2 } \\
\end{array}$}} & 20 & 80 & 0.10 & 3.336 & 9 & 2.989 & 13 & 3.739 & 6 & 3.317 & 9 & 0.188 & 1 \\
\hline & & 5 & 20 & 0.10 & 3.340 & 10 & 2.797 & 9 & 5.156 & 15 & 3.543 & 11 & 0.588 & 15 \\
\hline & & 10 & 40 & 0.20 & 3.415 & 11 & 3.165 & 17 & 3.719 & 5 & 3.446 & 10 & 0.193 & 2 \\
\hline & & 20 & 40 & 0.20 & 3.607 & 12 & 3.093 & 15 & 6.656 & 23 & 4.014 & 16 & 1.130 & 30 \\
\hline \multirow{4}{*}{$\begin{array}{l}\star \\
\star \\
\star\end{array}$} & DR2 & 5 & 50 & 0.10 & 4.043 & 18 & 2.963 & 12 & 5.537 & 16 & 3.969 & 15 & 0.733 & 21 \\
\hline & CMA & 5 & 10 & 0.10 & 4.256 & 21 & 3.835 & 24 & 4.970 & 12 & 4.275 & 20 & 0.266 & 6 \\
\hline & DR2 & 20 & 40 & 0.10 & 7.256 & 32 & 5.025 & 30 & 12.497 & 31 & 7.477 & 32 & 2.010 & 42 \\
\hline & DR2 & 1 & 10 & 0.20 & 9.886 & 35 & 7.269 & 35 & 12.513 & 32 & 9.993 & 35 & 1.669 & 40 \\
\hline \multirow[b]{4}{*}{$\star$} & DR2 & 1 & 10 & 0.10 & 10.308 & 36 & 7.254 & 34 & 13.978 & 35 & 10.168 & 36 & 1.974 & 41 \\
\hline & CMA & 1 & 10 & 0.10 & 11.282 & 37 & 8.758 & 39 & 16.961 & 38 & 12.198 & 37 & 2.336 & 44 \\
\hline & CMA & 1 & 10 & 0.20 & 13.553 & 38 & 9.254 & 40 & 15.974 & 37 & 13.032 & 38 & 2.195 & 43 \\
\hline & CMA & 20 & 40 & 0.01 & 16.967 & 42 & 14.955 & 45 & 18.409 & 40 & 16.747 & 40 & 1.030 & 28 \\
\hline
\end{tabular}


Table 4.2: Performance of initial step size values $\left(\sigma_{0}\right)$ (Column headings as in Table 4.1).

\begin{tabular}{|c|cc|cc|cc|cc|cc|}
\hline$\sigma_{0}$ & Median & Rank & Min & Rank & Max & Rk & Mean & Rank & Std & Rank \\
\hline 0.10 & 5.16 & 1 & 2.21 & 2 & 74.46 & 2 & 15.08 & 2 & 20.67 & 3 \\
0.20 & 5.52 & 2 & 2.07 & 1 & 72.40 & 1 & 14.75 & 1 & 20.13 & 1 \\
0.01 & 26.97 & 3 & 13.15 & 3 & 83.11 & 3 & 35.84 & 3 & 20.28 & 2 \\
\hline
\end{tabular}

Table 4.3: Performance of different parameter settings in laboratory experimentation. $\bar{L}$. denote average performance of laboratory experiments, $\bar{S}$. denotes average performance of simulation experiments. $\{\bar{S}, \bar{L}\}^{\star}$ denote the best average performance achieved with the respective adaptation scheme.

\begin{tabular}{|c|c|c|c|c|c|c|c|}
\hline Alg & $\mu$ & $\lambda$ & $\sigma_{0}$ & $\bar{L}$ & $\bar{S}$ & $\bar{L} / \bar{L}^{\star}$ & $\bar{S} / \bar{S}^{\star}$ \\
\hline CMA & 20 & 40 & 0.1 & 70.42 & 2.47 & 1.00 & 1.00 \\
CMA & 5 & 10 & 0.1 & 142.86 & 4.26 & 2.03 & 1.72 \\
CMA & 20 & 40 & 0.01 & 500.00 & 16.97 & 7.10 & 6.87 \\
\hline \hline DR2 & 20 & 80 & 0.2 & 156.25 & 2.70 & 1.00 & 1.00 \\
DR2 & 5 & 50 & 0.1 & 222.22 & 4.04 & 1.42 & 1.50 \\
DR2 & 20 & 40 & 0.1 & 250.00 & 7.26 & 1.60 & 2.68 \\
\hline
\end{tabular}





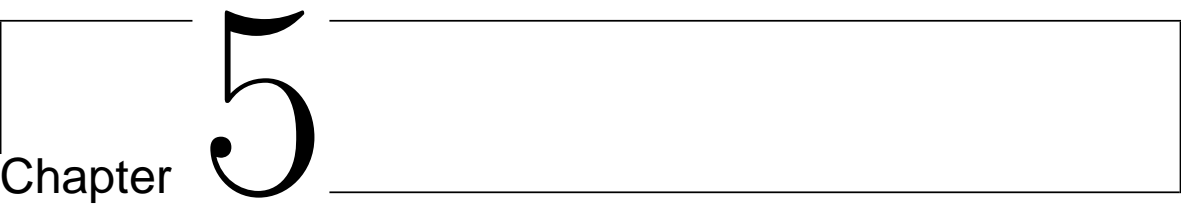

\section{Retracing pathways of evolutionary algorithms}

We have developed an experimental technique to expand upon optimisations using stochastic search methods. The technique is based on retracing the search path taken by a learning algorithm in a closed-loop optimisation experiment. In a study on the control of the energy-transfer process in an artificial light-harvesting complex, we use repeated measurements to disentangle two control mechanisms responsible for the learning curve. In another example, we use repeated measurements to compare fitness landscapes between variant systems. A solvent dependency of the fluorescence yield optimisation result in a model dye, Coumarin 6, reveals a linear trend in the control amplitude while preserving the general features of the learning curve. In addition, the repeatability of closed-loop optimisations is discussed. 


\subsection{Introduction}

Traditionally, the process of physical learning by experimental research proceeds with controlled experiments in which a problem of interest is studied as a function of one or more relevant experimental variables. The analysis of the experimental results then provides further understanding of the system by providing insights into the specific roles of these variables. One famous example of this approach is provided by Galileo. In his book 'Two new sciences' he demonstrates by a series of experiments how the distance travelled by a falling object is proportional to the square of the time [67]. This approach has led to a wealth of accumulated knowledge. However, as the systems grow in complexity, it becomes increasingly difficult to control all factors in an experiment, simply because the experimenter may not account for all factors. To establish causation the experimenter needs to have a control group where only one variable the variable of interest - is changed, and all other variables are held constant. The difficulty lies in how to control all other variables when there could potentially be an infinite number of variables.

Le Chatelier's principle can be used to further exemplify the issue of controlled variables. The principle states that "If a chemical system at equilibrium experiences a change in concentration, temperature, volume, or total pressure, then the equilibrium shifts to counteract the imposed change", and can be used in chemistry to predict the effect of a change in conditions on a chemical equilibrium; i.e. as a tool to explain and predict 'passive' control of the outcomes of reversible reactions. It has been successfully applied for instance in chemical industry to optimise product yields. However, couplings between variables, extra factors or additional changes in the conditions will cause such predictions to fail. One example of a complex system and failure to model it wrongfully assuming a set of controllable variables is shown in Figure 5.1. Modelling macroeconomical systems that have interdependent variables and in which organisations change, unexplored opportunities arise, and new choices are made, by using such models is not straightforward [68] and can ultimately lead to serious misjudgements. In general, applying simple principles like the one of Le Chatelier or using overly simplified models like the one in Figure 5.1 for systems that cannot be described by a set of independently controllable variables are doomed to provide an unrealistic picture of the system.

These days, such complex problems with large number of variables and unknown factors are encountered also frequently in natural sciences. Examples of such problems are numerous, including scientific, engineering and problems of commercial nature concerning product design and optimisation of industrial product lines, optical applications, acoustic problems, etc. etc [69-73]. In general, the realisation that a systematic variation of all parameters in a many-parameter phy sical system is not feasible has strongly motivated the use of alternative ways to aid in addressing these problems. Making approximations and conducting experiments with simplified model systems that mimick the physics of the real systems are common scientific methods to tackle this problem of complexity. Another approach to such multiparameter problems is to use stochastic search methods like evolutionary algorithms that allow for a large number of variables in finding the right combination to a given problem. 


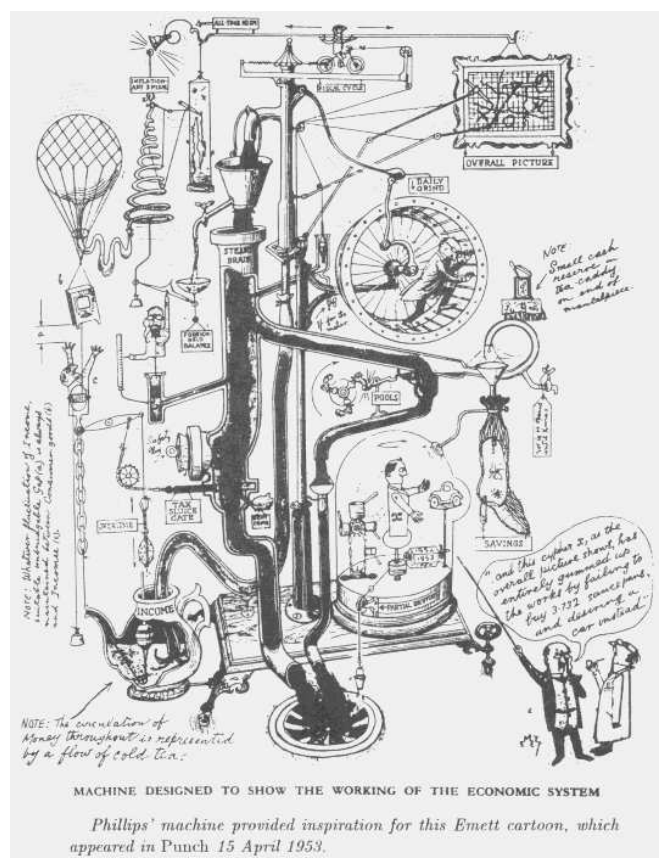

Figure 5.1: Moniac, a peculiar contraption that most resembles the work of a deranged plumber. Yellow tubes connect together a number of tanks and cisterns, around which coloured water can be pumped. Sluices and valves govern the flow of liquid and makeshift meters record the waterlevels. The plumber responsible for this device was William Phillips. His machine, first built in 1949 , is meant to demonstrate the circular flow of income in an economy. It shows how income is siphoned off by taxes, savings and imports, and how demand is re-injected via exports, public spending and investment. It is perhaps the most ingenious and best-loved of economists' big models. The figure shows a cartoonist's interpretation that appeared in Punch magazine 15 April 1953.

\section{The problem under study}

In this chapter we discuss the implications of using evolutionary algorithms in the field of laser control. Coherent control using laser light aims to steer physicochemical processes of atomic and molecular systems on the microscopic level [74]. For a limited number of simple systems, a driving field that takes the system from its initial state to the desired target state can be found by means of optimal control theory. However, in polyatomic systems insufficient explicit knowledge of the system Hamiltonian prohibits calculation of the optimal control field. To resolve this problem, a practical approach incorporating adaptive pulse shaping in a closed loop guided by a learning algorithm was developed [8]. This learning-loop control technique lets a physical quantum system solve its own Schrödinger equation in real time in laboratory, thus evading the problem of inaccuracies in a priori calculations due to inevitable approximations. Today, optimal control using adaptive learning-loop technique is well established and the number of systems brought under control is impressive and rapidly 
increasing $[75,76]$.

Performing such black-box experiments, however, comes with certain drawbacks. First, realising control by closed-loop optimisations provides but little knowledge about the instigating mechanisms behind the control. Therefore, it is possible to reach the control objective without actually learning anything new about the system [10]. It can be argued that a successful optimisation is only completed by solving of the corresponding problem of inversion. Thus, to resolve the necessary and/or essential control knobs and their settings, more often than not extra measurements are needed as discussed in Section 2.5. The second drawback relates to the reproducibility of the experiments. One key characteristic of the learning algorithms used in the closedloop optimisations is that, unlike traditional search algorithms relying on derivative methods, they are indeterministic. In principle, this makes it impossible to 'repeat' an optimisation since there is no guarantee that the algorithm will follow the same path on the multidimensional fitness landscape. This disagrees strongly with one of the basic methodological principles of experimental science: the measurements must be reproducible.

In this chapter we propose a simple idea on how to expand upon closed-loop experiments by retracing the pathways taken by the algorithm in a repeated measurement. First, we demonstrate how the aforementioned drawback of reproducibility can be avoided and the repeatability of results can be verified. Secondly, we show how the found control results can be further utilised. A closed-loop optimisation actually provides us a limited map of the fitness landscape. Reusing the obtained optimisation results by retracing the pathway in a series of measurements using a set of controlled variables can provide insights to the importance and the influence of a particular variables to the control. Hence, the repeated measurements can actually be used to bring optimal control results from the learning-loop approach back to the realm of Galilean hard science. The technique is demonstrated by two case studies: The control of energy flow in an artificial light-harvesting complex, and control of emission yield of Coumarin 6. However, we emphasise that this technique is general and can be applied to the result of any optimisation involving a stochastic search.

\subsection{Experimental}

The experiments make use of a pump-probe setup with a tailored pump pulse and an unmodulated probe pulse as described in Chapter 3. In the energy-flow study, the pump spectrum was tuned to the first optically allowed transition $\left(S_{0} \rightarrow S_{2}\right.$, Figure 5.2) of the carotenoid moiety of the artificial light-harvesting complex. In the solvation study the pump spectrum was located at the red side of the linear absorption band of Coumarin 6 dye overlapping with the emission band of the dye. In these studies only phase shaping was used.

The experiments begin by recording a successful learning curve in a learning-loop optimisation experiment using an evolutionary search algorithm. During the optimisations, the best pulse shape out of each generation is saved allowing us to remeasure the obtained learning curve, which is then subsequently compared to the original. 


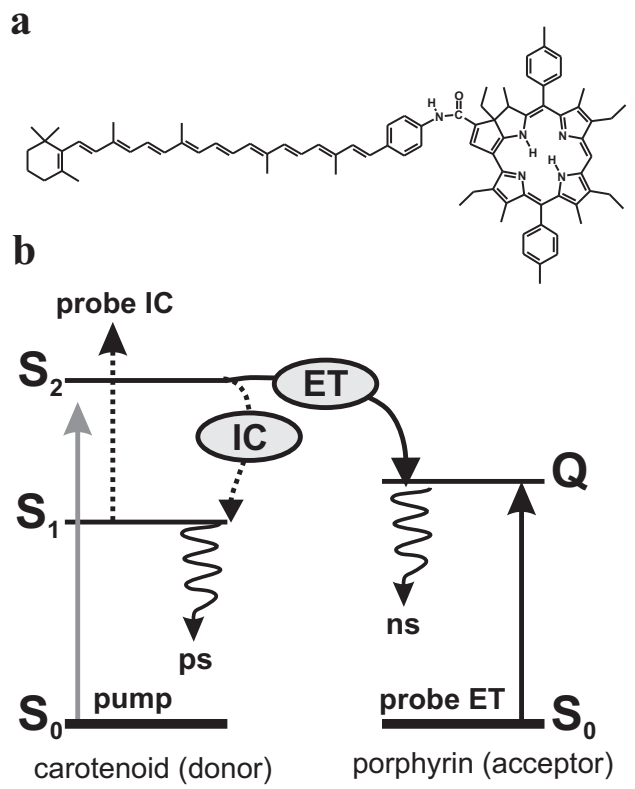

Figure 5.2: Structure (a) and the simplified energy-level diagram (b) of the artificial lightharvesting dyad.

\subsection{Results and discussion}

\subsubsection{Energy transfer yield}

The first sample is an artificial light-harvesting complex that closely mimics the earlytime photophysics of the LH2 complex [77, 78]. The artificial light-harvesting complex consists of a single donor (carotenoid) and single acceptor (porphyrin) moiety [77] (see Figure 5.2a), thus reducing the structural complexity significantly compared to LH2 [79]. In the original optimal control experiments the target objective was to manipulate the branching ratio between the functional energy transfer (ET) channel and the internal conversion (IC) loss channel. The signals belonging to ET and IC are resolved from the transient spectrum measured at 8 ps time delay and used as feedback for the optimisation (see Figure 5.2b, and for more details Chapter 6).

Figure 5.3 shows the learning curve from the original optimisation (filled circles) having a total increase of the IC/ET ratio of some $20 \%$ : an initial jump of $\sim 10 \%$ and a gradual learning part providing further $10 \%$ improvement. The repeated measurement recorded two days later (open squares) shows a good match with the obtained result thus verifying the repeatability of the obtained result.

The learning curve presented here is one of many closed-loop optimisations on the ratio IC/ET, all sharing two characteristic features. As in Figure 5.3, we invariably observed an initial 'jump' after which, if the optimisation was successful, a gradual rise to a further enhancement of some degree. To better understand these common characteristics of the obtained learning curves we now proceed with simulations and 


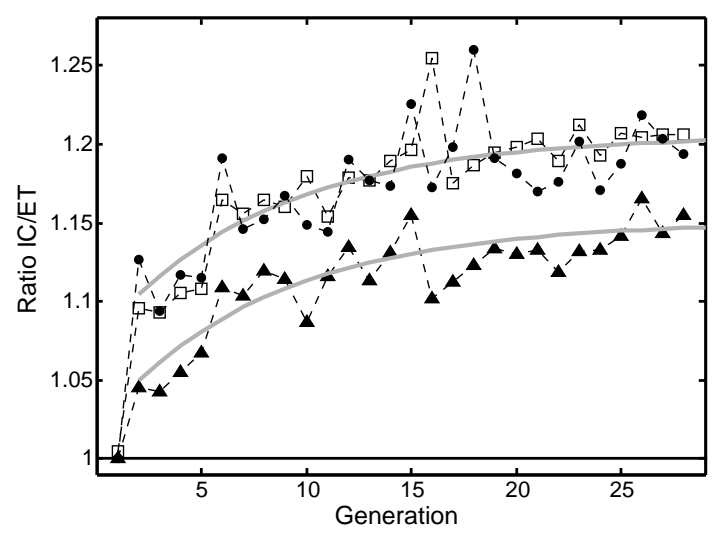

Figure 5.3: Original optimisation and the repeated experiments of the IC/ET ratio in artificial light harvesting complex. Original (filled circles) repeated same power (open squares), and repeated with half of the laser fluence (filled triangles). The grey lines show the fit for the repeated measurements with full and half power, normalised to the first generation

repeated measurements.

The optimal control experiments were done using laser fluences that partially saturate the carotenoid $\mathrm{S}_{0} \rightarrow \mathrm{S}_{2}$ transition when using a short unshaped pulse. Therefore, we wanted to simulate if this saturation plays a role in the measured IC and ET signals and/or in the control results. In general, using a pulse shape that is stretched in time will give the photons in the trailing end of the pulse a probability to re-excite molecules that have had time to relax back to the ground state (see Figure 5.2). In the case of signal saturation, the number of excitation processes can this way be increased leading to an increase in the measured time-integrated signal compared to the short pulse. Since the pulse analysis of the optimisations revealed a pulse-train structure we simulated the magnitude of the IC and ET signals using a pulse train with 7 subpulses together with the experimental saturation curves of IC and ET (Figure 5.4, inset). Note, that the same transition (carotenoid $S_{0} \rightarrow S_{2}$ ) is responsible for the saturation of both signals and an increase in the time-integrated signal of IC would be matched with an increase of ET. Nevertheless, Figure 5.4 shows that, the ratio IC/ET increases as a function of the subpulse spacing. At the intensity where the original optimisation was made, the jump is approximately $10 \%$ for a total pulse time span (= subpulse spacing $\times$ number of pulses) of few picoseconds, which is an average time stretch for a random pulse with the used experimental parameters. The increase originates from the difference between the lifetimes of the two signals. In summary, assuming partial saturation, and compared to the TL pulse, the pulse stretch essentially causes a time shift for the 'free' evolution of the signals: the long-lived ET is invariable to this time shift at $8 \mathrm{ps}$, whereas IC shows an increase.

To verify that a trivial mechanism that avoids the partial saturation by stretching the pulse in time is behind the observed jump in the learning curves and, more 


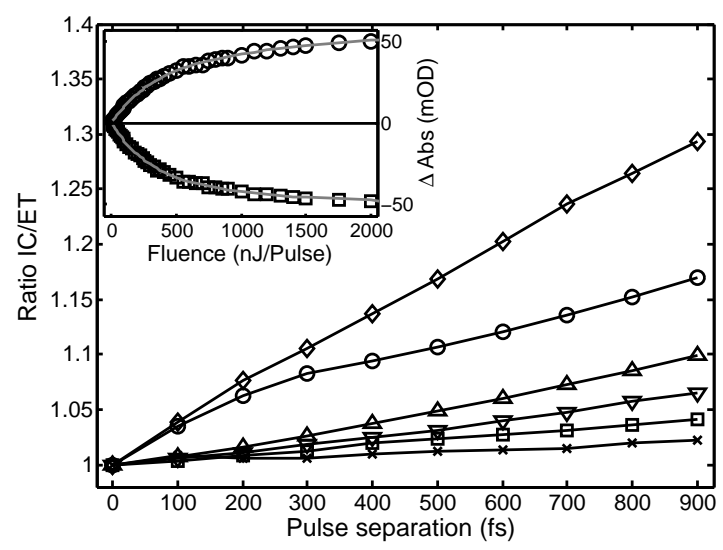

Figure 5.4: Simulated IC/ET ratio taking saturation into account. Ratio IC/ET as a function of pulse separation with increasing pulse pulse energies: 50, 100, 250, 500, 1000, and 2000 $\mathrm{nJ} /$ Pulse (asterisk, square, delta, triangle, circle, and diamond, respectively). Inset: Experimental saturation curves for IC (circle) and ET (square) together with the exponential fit (grey lines).

importantly, is not the cause of the subsequent gradual growth of the IC/ET ratio, we conducted repeated measurements. The recorded pulse shapes from the IC/ET optimisation were tested with the full fluence (Figure 5.3, open squares) used in the original optimisation as well as only half the fluence (Figure 5.3, filled triangles). Data from the repeated measurements was fitted with a simple exponential function, showing how the same curve fits both measurements, when normalising to the first generation (Figure 5.3, grey lines). This shows how the jump and the 'learning' part have different fluence dependencies: the initial jump decreases to approximately half of its amplitude whereas the learning part amplitude remains the same $(\sim 10 \%)$. We conclude that the initial jump observed in the optimisations is due to a trivial and incoherent control mechanism that merely avoids saturation, and that the learning originates from an active control mechanism over the branching of the energy flow in the dyad which can be separated from the trivial control mechanism.

In summary, using the energy transfer example we demonstrated how the retracing technique can be used to overcome the drawback related to the reproducibility of experiments. By repeating the recorded pulse shapes this ambiguity is avoided and results can be directly compared. To verify the repeatability and robustness of optimal control results, we further showed how it is possible to disentangle two different mechanisms underlying the fitness curve by monitoring how the shape of the learning curve reacts to the varying pulse intensity. This elucidates the power of repeated measurements as a complementary technique to learning-loop experiments. 


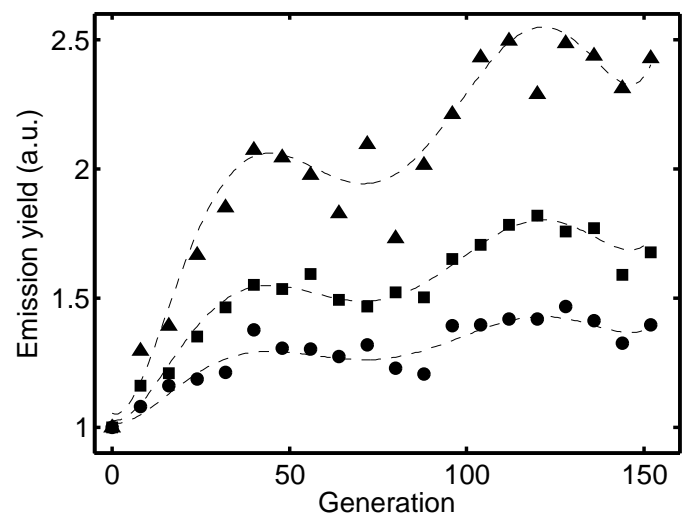

Figure 5.5: Normalised emission yield curves for Coumarin 6 with different solvents hexane (triangles), octane (squares), and decane (circles). The dashed line in all six curves is the same polynomial function only scaled in amplitude.

\subsubsection{Emission yield}

In the second example, we demonstrate how repeated measurements can be used to explore fitness landscapes. We study the effect of the solvent on a control experiment by repeating a learning curve obtained by closed-loop optimisations on Coumarin 6 fluorescence yield and varying the solvent. The original optimisation was made in cyclohexane where the feedback signal was stimulated emission probed by a whitelight pulse at $1 \mathrm{~ns}$ delay. In the repeated measurements we used a range of linear alkanes (hexane, octane and decane) as solvents.

To reduce the experimental time, the remeasured fitness curves were undersampled by only using the pulse shape from every 8th generation. Multiple runs at varying laser intensities were performed to check the power dependence of the optimisation result. The fitness curves for the repeated optimisations in the different solvents were normalised to the fitness of the transform-limited pulse, allowing for direct comparison. The resulting curves were fit with a polynomial function that maps out the ridges and valleys in the traversed fitness landscape. Remarkably, the same polynomial shape fits all curves; only a scaling factor is adjusted. Hence, we conclude that the fitness landscapes of the dye in the different solvents are comparable. The magnitude of the scaling factor shows how well the system can be controlled in the corresponding solvent. We indeed see that the ability to control the intensity of the stimulated emission strongly depends on the solvent. The enhancement of the stimulated emission compared to excitation with the transform limited pulse varies from $40 \%$ in decane to $145 \%$ in hexane.

We stress that due to the indeterministic nature of evolutionary algorithms, this trend could only be found with the use of repeated measurements of a single lear- 
ning curve. Further, the repeated curves indicate that the fitness landscape is the same across all systems and that the result of the optimal pulse shape can be directly compared. Although in some cases comparing the transform limited (TL) pulse to the obtained best pulse may suffice and bring further understanding of the control [14] we have shown that also the shape of the learning curve is significant: The saved pulse shapes map out the path through the multidimensional search space and the fitness curve depicts the value of a chosen physical parameter along this path. Generally, variations in the shape of the fitness curve in the repeated experiments indicate that the fitness landscape is different, and therefore the underlying physics have changed. It is hence essential to retrace the shape of the learning curve, as changing a parameter might still result in a different value between TL and the best found pulse, but perhaps not due to the same mechanism.

\subsection{Conclusions}

We have presented a simple technique that complements adaptive femtosecond control experiments and can ultimately be used to utilise the obtained optimisation results further. The power of repeated measurements is demonstrated with two examples, and the results show how the technique allows for a direct comparison between the results when a parameter is systematically varied. The repeated measurements allow for the quantification of the robustness of optimisation results. Furthermore, by reapplying recorded pulse shapes along the learning curve, it is possible to systematically elucidate the influence of a single parameter (laser fluence, solvent viscosity, temperature, pressure, etc.). 



\section{Part II}

\section{Applications}





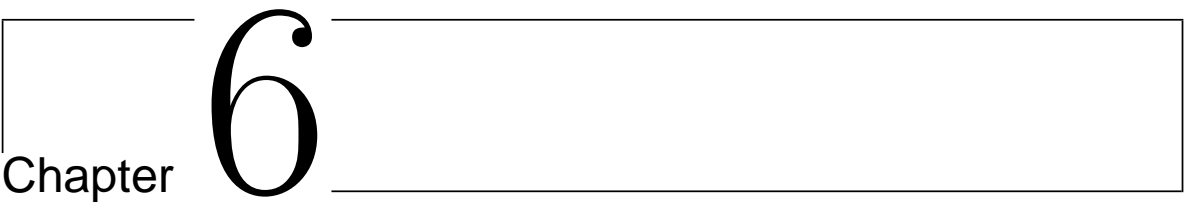

\section{Ultrafast energy transfer dynamics of a bioinspired dyad molecule}

A carotenopurpurin dyad molecule was studied by steady-state and pump-probe spectroscopies to resolve the excited-state deactivation dynamics of the different energy levels as well as the connecting energy flow pathways and corresponding rate constants. The data were analysed with a two-step multi-parameter global fitting procedure that makes use of an evolutionary algorithm. We found that following ultrafast excitation of the donor (carotenoid) chromophore to its $\mathrm{S}_{2}$ state, the energy flows via two channels: energy transfer $(70 \%)$ and internal conversion $(30 \%)$ with time constants of 54 and $110 \mathrm{fs}$, respectively. Additionally, some of the initial excitation is found to populate the hot ground state, revealing another limitation to the functional efficiency. At later times, a back transfer occurs from the purpurin to the carotenoid triplet state in nanosecond timescales. Details of the energy flow within the dyad as well as species associated spectra are disentangled for all excited-state and groundstate species for the first time. We also observe oscillations with the most pronounced peak on the Fourier transform spectrum having a frequency of $530 \mathrm{~cm}^{-1}$. The dyad mimics the dynamics of the natural light-harvesting complex LH2 from Rhodopseudomonas acidophila and is hence a good model system to be used in studies aimed to further explain previous work in which the branching ratio between the competing pathways of energy loss and energy transfer could be manipulated by adaptive femtosecond pulse shaping. 
$\mathbf{a}$
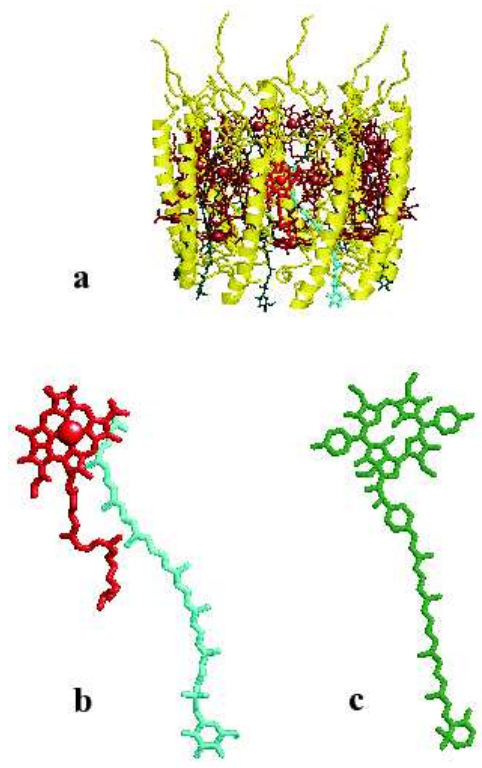

Figure 6.1: Structure of LH2 pigments as compared to the dyad. Panel a: LH2. Panel b: expansion of two highlighted pigments in Panel a (the carotenoid donor in blue and the porphyrin acceptor in red). Panel c: schematic structure of the carotenopurpurin dyad; the donor and acceptor are chemically bridged by an amide link.

\subsection{Introduction}

In photosynthetic plants and algae, the early time energy transfer (ET) between two chromophores (from carotenoid molecules to the porphyrin molecules) is the key step in making use of the energy of the blue-green photons of sun light in photosynthesis [80]. In a photosynthetic light-harvesting complex of Rhodopseudomonas acidophila, a photosynthetic purple bacteria (Figure 6.1a), energy is transferred from rhodopin glucoside carotenoids to bacteriochlorophyll $a$ molecules. In this particular system, the efficiency of ET from the donor (carotenoid) to the acceptor (bacteriochlorophyll) is about $56 \%$, while the rest of the harvested energy is dissipated via the intramolecular process of internal conversion (IC) [81-83]. Previously, Herek and co-workers demonstrated that, by using adaptive femtosecond pulse shaping, it is possible to attain coherent control over the branching ratio between the two competing pathways [43]. The relative efficiencies of the two processes, the functional pathway (energy transfer, ET) and the loss channel (internal conversion, IC), could be manipulated by $30 \%$. The features of the potential energy surfaces, and the detailed pathways of the energy flow in the natural light-harvesting complex LH2, are critical factors in understanding the mechanisms leading to this control. However, this system is comprised of several chromophores of both types, embedded in a large protein, and the size becomes a problem in describing the system by means of molecular modelling. In this work, to better understand the mechanism of coherent control, we proposed an ex- 


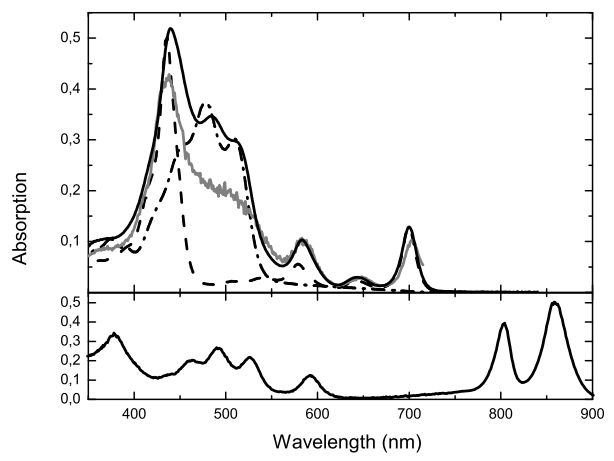

Figure 6.2: Upper panel: absorption spectra of the dyad (solid line), carotenoid (dashed-dotted line), purpurin (dashed line), and excitation spectrum of the dyad (grey line). Lower panel: absorption spectrum of the LH2 complex from $R$. acidophila.

perimental method to investigate the mechanisms behind the LH2 control study by controlling a simpler model system, comprised of only an acceptor and a donor molecule [84]. As a preparatory step to the coherent-control experiments we performed a detailed ultrafast transient-absorption study of a caroteno-purpurin dyad model system (Figure 6.1c), and compared the structure, spectra, and dynamics to that of LH2. We begin with a structural description of LH2 and the dyad. The crystal structure of the LH2 has already been determined to an atomic resolution [79]. LH2 consists of nine subunits, arranged in a ring structure, each including three bacteriochlorophyll (Bchl) molecules and one rhodopin glucoside carotenoid (Car) molecule, all embedded and noncovalently bound to the surrounding protein. The Bchl molecules are arranged in two rings that are physically and spectrally different. According to the maxima of their $\mathrm{Q}_{y}$ absorption peak, they are called the B800 and B850 BChls. Each carotenoid has an S-like conformation and interacts with the neighbouring Bchls in two protomers. The distance from the carotenoid $\pi$-conjugated stem to the Bchl-B800 macro cycle is $\sim 3.4 \AA$, and $\sim 3.6 \AA$ to the Bchl-B850. In comparison, the dyad has only a single carotenoid and a single purpurin, covalently linked together by an amide group (Figure 6.1c). The distance from the edge of the pi-conjugation of the carotenoid to the edge of the pi-conjugation of the purpurin ring is $\sim 3.7 \AA$.

Despite these structural dissimilarities, the dyad mimics the major spectral features of the LH2 complex well. The steady-state spectra of both systems consist of overlapping carotenoid and purpurin (Bchl in LH2) bands, depicting their well-known features (see Figure 6.2). Both LH2 and the dyad show a characteristic three-peak structure in the region of $400-550 \mathrm{~nm}$, corresponding to the carotenoid absorption. The peak of the $0-0$ vibrational band of the carotenoid $S_{2}$ state in the dyad $(\sim 520 \mathrm{~nm})$ is slightly blue shifted relative to that of LH2 $(\sim 525 \mathrm{~nm})$, and the same trend is seen in the purpurin/Bchl $\mathrm{Q}_{x}$ bands (583 nm vs. $590 \mathrm{~nm}$ ). In LH2, the position of the Bchl $\mathrm{Q}_{y}$ band is strongly dependent on the embedding of the pigment in the two distinct 
binding sites of the protein ( 800 or $850 \mathrm{~nm}$ ). Further, excitonic coupling in the B850 ring affects the line shape of the absorption band [81]. In solution the difference is much less: Bchl absorbs at $\sim 770 \mathrm{~nm}$, while the purpurin tetrapyrrole is at $\sim 700 \mathrm{~nm}$. More important is that the $\mathrm{Q}_{x}$ bands, which are primarily involved in the ET process from the carotenoids (see below), are overlapping.

To probe the degree of similarity between the LH2 and the dyad, we focused on the function of LH2 as a light-harvesting complex. Only by showing a similarity in the internal dynamics of ET in the two molecules, can we really conclude that our model system is a good candidate for investigating the previous results of coherent control. Critical in this analysis are details of carotenoid photophysics, that were proposed to play a crucial role in the control experiments in LH2 [44]. Hence, we made it our priority to identify the spectral signatures belonging to the different species involved in the dynamics and to understand the pathways, and their efficiencies, related to the ET from the carotenoid to the purpurin.

In the case of LH2, the pathways and related rate constants have been previously described in detail by many studies $[81,82,85]$. According to the generally accepted energy-flow model, about $51 \%$ of the absorbed energy in this particular lightharvesting complex is transferred to the accepting Bchl states (to B850 Bchls in about $190 \mathrm{fs}$, and to B800 Bchls in about $280 \mathrm{fs}$ ). The ET process can be described to be first-order by a Förster mechanism [81]. The rest of the energy is from the carotenoid $\mathrm{S}_{2}$ state and is dissipated via intramolecular vibrational redistribution within about 120 fs down to the so-called dark $S_{1}$ excited electronic state of the carotenoid. This process is enhanced by a conical intersection between the $S_{2}$ and the $S_{1}$ states of the carotenoid [86]. In the LH2 complex, about $20 \%$ of the energy is transferred from the carotenoid $\mathrm{S}_{2}$ state to the B800 Bchls, and about $31 \%$ to the B850 Bchls. The energy transferred to B800 will subsequently arrive to the B850 ring Bchls. In addition, a small amount of the energy (about $5 \%$ ) is transferred from the $\mathrm{S}_{1}$ state of the carotenoids to the B800 Bchls, giving a total efficiency of about $56 \%$ for the ET from the carotenoid to the Bhcls [82].

The biomimetic dyad molecule has been characterised previously by Macpherson et al., by using pump-probe, fluorescence up-conversion, and time correlated single photon counting techniques [77]. Results show that in the dyad, approximately $70 \%$ of the energy is transferred to the purpurin moiety after excitation of the carotenoid portion of the molecule with a $488 \mathrm{~nm}$ laser pulse. According to their analysis, all of the energy is transferred from the $S_{2}$ state of the carotenoid. Unfortunately, the time resolution in these experiments was about 3 times longer than the fastest resolved rate constant. In similar systems, it was shown that some of the energy is also transferred from the $S_{1}$ state of the carotenoid, depending on the conjugation length of the carotenoid [87]. Since the publication of ref [77] in 2002, considerable interest in carotenoid reaction dynamics has shifted to the presence and role of additional (dark) states (i.e., the hot ground state or fleeting intermediate states) [88-90]. To date, no discussion on the involvement of the hot ground state or additional carotenoid excited states and their role in the photophysics of the dyad has been presented, nor has any previous study extracted the spectra belonging to the different spectral species involved in the dyad photophysics.

In the LH2 coherent-control experiments, a possible mechanism is the activation of 
a backbone vibrational mode of the carotenoid favouring the loss channel by directing the wave packet on the excited state toward the conical intersection between $S_{2}$ and $S_{1}$ states [44]. Carotenoid molecules in LH2 are embedded in the protein by noncovalent interactions, which may lead to augmentation of the importance of such skeletal modes on the branching of the energy flow. This raises the question as to whether the dyad exhibits similar low-frequency modes, and if so, what role might they play in the early time photophysics? Further, is the efficiency of ET from the carotenoid to the purpurin in the dyad in solution dependent on these modes? Is it possible to activate such modes of the dyad by impulsive Raman scattering (IRS) as is the case in the LH2 complex?

In the coherent-control experiments, the feedback signal from the molecule will be resolved by means of pump-probe spectroscopy. The resulting transient-absorption spectra are a sum of many overlapping signals, originating from excited-state absorption, stimulated emission, and bleach signals of different spectral species, each depicting unique spectral and temporal characteristics. Previously, the data analysis of the pump-probe data was based on so-called single trace fitting [77], and no global analysis of transient-absorption data has been reported on this system. A thorough understanding of the interplay of different signals contributing to the transient absorption is essential for any future studies that utilise these signals. For example, for the purposes of the coherent-control experiments, it is important to extract a reliable feedback signal that correlates with the process(es) one aims to control. In this study, we used a global fitting procedure to unravel the complex data by using a spectro-temporal model [91].

The caroteno-purpurin dyad (Figure 6.1) is a model system that mimics the salient features of the natural photosynthetic complex well, while preserving structural simplicity. A complete/global characterisation of the photophysics of this system is described in this article; it will be advantageous in the interpretation of the coherentcontrol experiments.

\subsection{Experimental procedures}

The caroteno-purpurin dyad was prepared according to published procedures [77]. Steady-state absorption spectra were measured with a Jasco V-530 spectrophotometer, with the sample in a $0.5 \mathrm{~mm}$ rotating cuvette (built in-house) used in the pump-probe experiments. Corrected fluorescence excitation and emission spectra were obtained using a SPEX Fluorolog 112 and optically dilute samples $(A<0.07$ in toluene). The excitation spectrum was corrected using a correction file obtained with the parent purpurin methyl ester by assuming that the absorption and fluorescence excitation spectra were identical [79].

The pump-probe setup was as follows: Part of the output of an amplified Ti:sapphire (Clark CPA-2001) laser was coupled into a non-collinear optical parametric amplifier (NOPA), which produced $\sim 10 \mu \mathrm{J}$ near-transform-limited pulses at $510 \mathrm{~nm}$ with $\sim 28 \mathrm{~nm}$ fwhm and $18 \mathrm{fs}$ pulse duration; these pulses were used as the pump pulses. A small fraction of the residual fundamental light was focused to a $2 \mathrm{~mm}$ sapphire window to create a white light continuum (WLC) that provided spectrally broad probe pulses ranging from 450 to $710 \mathrm{~nm}$. To avoid any anisotropy effects, the polarisation angle between the pump and the probe pulses was set to the magic angle $\left(54.7^{\circ}\right)$. The 
two beams were focused and overlapped at the sample position, where the fwhm of the Gaussian intensity profile of the pump beam was $250 \mu \mathrm{m}$. The pulse energy was set to $100 \mathrm{~nJ}$ with an adjustable filter, giving $\sim 5 \times 10^{14}$ photons $\mathrm{cm}^{-2}$. The sample cuvette was rotated sufficiently quickly to provide a fresh sample for every pulse to avoid sample degradation or the accumulation of long-living states. The probe pulses were coupled into a spectrograph (Acton), and individual spectral components were focused onto a 256 pixel diode array. Diode signals were read out and AD converted at a rate of $1 \mathrm{kHz}$, thus obtaining shot-to-shot statistics for each measured spectra. Spectral resolution of the detection system was approximately $1 \mathrm{~nm} / \mathrm{pixel}$.

The time resolution of the pump-probe experiments as well as the amount of spectral dispersion in the WLC was determined by measuring the sum-frequency-mixing signal of the pump and probe pulses at the sample position in a $25 \mu \mathrm{m}$ thick BBO crystal. The wavelength to be mixed from WLC was selected by tuning the phase matching angle of the crystal; in this way, the mixing of different wavelengths of the WLC could be measured. The time resolution was $\sim 70$ fs across the spectrum, and the overall time delay between the blue and the red parts of the WLC spectrum (chirp) was approximately $\sim 300$ fs. The measured chirp curve was used to remove the WLC dispersion from the data prior to analysis.

Spectroscopy-grade toluene was purchased from Riedel-DeHan and used without further purification. The optical density used in the pump-probe experiments was $0.3 \mathrm{OD}$ at $510 \mathrm{~nm}$ in the $0.5 \mathrm{~mm}$ path-length rotating cuvette. To check for any sample degradation, the steady-state absorption spectrum was measured before and after the measurements. No changes in the OD or spectral shapes were observed, indicating sample stability. All measurements were performed at room temperature.

\subsubsection{Data analysis}

Temporally and spectrally resolved data were analysed globally using analysis software developed in-house. The principles of the method were first described by Holzwarth [91] and further refined and used by van Grondelle's group [92]. Recently, a development adding the use of evolutionary algorithms to unravel the deactivation pathways in LH2 of Rhodopseudomonas acidophila was reported by Wohlleben et al [93]. Here, we further extend this approach by introducing a powerful covariancematrix adaptation of an evolutionary algorithm allowing a large number of parameters $[35,94]$.

In the first step, the data are fitted using a so-called sequential model where the 2D surface is described by a number of evolution associated difference spectra (EADS) that evolve sequentially and irreversibly from one to another. The free parameters in this step are the rate constants (i.e., the lifetimes) of each EADS and their spectral shapes. This provides us with preliminary information on the timescales that are involved in the kinetics as well as where they are represented spectrally.

In the second step, various, more elaborate models are tested (target analysis). The goal of the target analysis is to resolve the SAS that belong to each excited state that depicts a transient spectrum, and the rate constants and the deactivation pathways connecting these states. These models are comprised of parameters for the initial distribution of population (states (de)populated by the excitation pulse), rate constants 
connecting the states and species associated spectra (SAS). Ground-state bleach signals are described by inverted absorption spectra, having only their amplitude as a free parameter. The spectral profiles of the rest of the SAS were each described by 50 parameters, using a small weighting factor, based on the second derivative of the spectra, to favour smooth spectral shapes.

The fitting proceeds within a learning loop, where the target objective is to find the spectro-temporal model that, together with corresponding rate constants, best fits the data. The quality of the fit is evaluated by its $\chi^{2}$ value. The program employs an algorithm based on evolutionary principles. We start with a generation of 20 random sets of parameters, corresponding to 20 simulated surfaces, comparing the surfaces to the measured data, and evaluating their $\chi^{2}$ values. On the basis of this information, a new generation of individuals is created by the algorithm. The iteration is then left to run until convergence to an acceptable $\chi^{2}$ value is reached and the measured data are simulated sufficiently. Details of the fitting program and examples will be presented elsewhere. The errors in the resolved time constants (and quantum yields) were determined using the variance in several fits resulting in comparable $\chi^{2}$ values.

\subsection{Results}

\subsubsection{Steady-state measurements}

Absorption spectra of the dyad, the reference carotenoid, and the reference purpurin (all dissolved in toluene) are shown in Figure 6.2, along with the LH2 spectrum for comparison. Essentially, the dyad spectrum is a sum of the absorption of the two contributing chromophores, showing the first optically allowed transition of the carotenoid from the ground state to the $S_{2}$ state between 350 and $550 \mathrm{~nm}$ with its vibrational substructure, as well as the Sôret, $\mathrm{Q}_{x}$ and $\mathrm{Q}_{y}$ absorption bands of the purpurin with maxima at 439, 583, and $699 \mathrm{~nm}$, respectively. In the dyad the amplitude of the $\mathrm{Q}_{x}$ band is slightly enhanced and shifted $5 \mathrm{~nm}$ to the red as compared to the isolated purpurin in solution. Also, the Sôret band shows a minor red shift in the dyad. The vibrational substructure in the absorption of the carotenoid moiety is reduced as compared to the free Car in solution.

The fluorescence excitation spectrum of the dyad was measured to determine the efficiency of the singlet-singlet ET. Figure 6.2 shows the fluorescence excitation spectrum of the dyad, normalised with the linear absorption spectrum at the maximum of the $\mathrm{Q}_{x}$ band $(583 \mathrm{~nm})$, where all of the absorption is due to the purpurin. From the ratio between these normalised spectra, in the region where the carotenoid absorption dominates (460-515 $\mathrm{nm}$ ), the yield of the ET is on average $67 \%$.

\subsubsection{Time-resolved measurements}

To characterise the energy flow pathways following the excitation by the pump pulse, we performed spectrally resolved ultrafast transient-absorption measurements, where the time delay between pump and probe pulses was scanned from negative times to $6.2 \mathrm{~ns}$ and the broad band probe light was detected from 470 to $710 \mathrm{~nm}$. The resulting 2-D pump-probe data are shown in Figure 6.3. After the excitation, five major contributions are visible. At early times, the negative signal on the blue side of the measured 


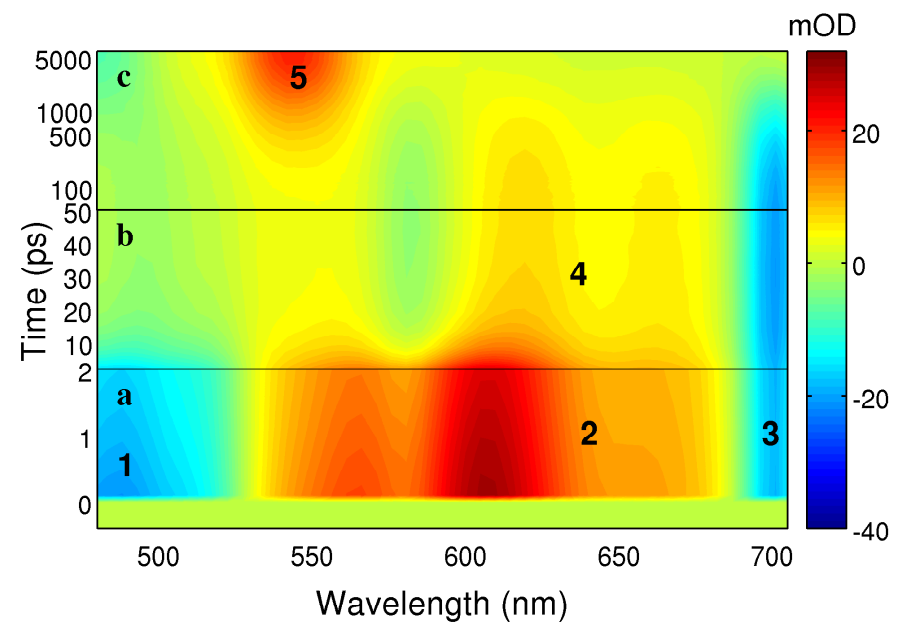

Figure 6.3: Pump-probe data as a function of time (y) and wavelength (x). The characteristic

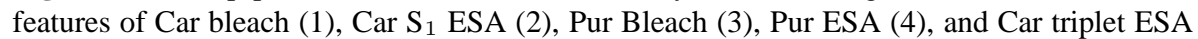
(5) are indicated. Note the three different time axes. a: 0-2 ps, linear; b: 2-50 ps, linear; and c: 50 ps to 6.2 ns (logarithmic).

spectral range coincides spectrally with the carotenoid steady-sate absorption (1); the strong positive signal in the central region of the measured spectral range (2) is due to an excited-state absorption; and the negative signal at $\sim 700 \mathrm{~nm}$, originates from the bleach of the $\mathrm{Q}_{y}$ transition of the purpurin moiety (3). Further, the bleach signal due to the $\mathrm{Q}_{x}$ band of the purpurin is seen as a crevice carved at $580 \mathrm{~nm}$ on the positive excited-state absorption extending from 520 to $690 \mathrm{~nm}$.

A major part of the positive excited state absorption (ESA) as well as the carotenoid bleach signal decays during the first 20 ps. A broad and weak positive signal in the central region remains together with the features of the purpurin bleach (4). A small rise of the negative signal at $700 \mathrm{~nm}$ can be seen. At the later times, as the positive signal between 600 and $690 \mathrm{~nm}$ and the negative signal at $700 \mathrm{~nm}$ both decay on a nanosecond timescales, a new positive band arises, peaking at $540 \mathrm{~nm}$ (5). This is accompanied by a slight increase of the negative signal on the blue side of the measured spectrum (Figure 6.3c, top left corner).

It is evident that various signals are superimposed. This congestion and/or overlap of signals causes difficulties in the data analysis. For example, the so-called singletrace fitting results in a multi-exponential function, of which physical interpretation may become an intractable problem [91, 92]. To extract quantitative and physically interpretable information out of the measured pump-probe data, we made use of a global fitting procedure described in the Experimental Procedures.

To determine the number of time constants involved in the overall spectral evolution, the data were fitted using a simple sequential model. The model consists of a number of states evolving subsequently to the next one with time. The related spectra are left to develop without any constraints as the fitting proceeds. In principle, 


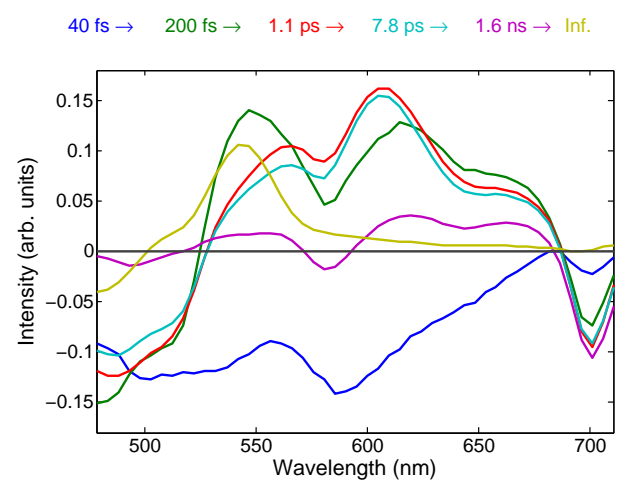

Figure 6.4: Results of the global analysis using the sequential model. The following EADS are shown: 40 fs (dark blue), $200 \mathrm{fs}$ (green), $1.1 \mathrm{ps}$ (red), $7.8 \mathrm{ps}$ (light blue), $1.58 \mathrm{~ns}$ (magenta), and inf. (beige)

this approach is purely mathematical, and requires no physical intuition or previous knowledge of the system, both of which may well be a hindrance to an objective data analysis. Hence, this approach provides a beneficial way to begin to unravel the kinetics of any molecular system. In this first step, the pump-probe surface is expressed by so-called evolution-associated difference spectra (EADS) and the corresponding lifetimes that link the spectra together (e.g., the third EADS rises with the second lifetime and decays with the third). The found EADS (Figure 6.4) are used in concert with the experimental data to provide the first insights into the kinetics of the dyad molecule. To aid the description of the rich dynamics, depicting features ranging from femtosecond to nanosecond timescales, the data are presented here in three different time regimes: early ( $0-2 \mathrm{ps}$ ), medium (2-50 ps), and late time ranges (50 ps to $6.2 \mathrm{~ns}$ ). The pump-probe surface and the temporal cuts are plotted accordingly (regions a, b, and $\mathrm{c}$ in Figure 6.3 and Figure 6.6). From the pump-probe surface (Figure 6.3), accompanying spectral and temporal sections (Figure 6.5 and Figure 6.6) as well as EADS (Figure 6.4), the following characteristic temporal and spectral features, are qualitatively recognisable.

Early time range. Located spectrally in the blue end (from 480 to $525 \mathrm{~nm}$ ) lies the instantaneous negative carotenoid bleach $\left(\mathrm{Bl}_{\mathrm{Car}}\right)$ signal, which is partially overlapped by the negative signal originating from the stimulated emission (SE) from the carotenoid $\mathrm{S}_{2}$ state between 500 and $620 \mathrm{~nm}$ (Figure 6.4, blue line). The first EADS lives only some tens of femtoseconds before the strong positive carotenoid $S_{1} \rightarrow S_{n}$ ESA dominates. This broad band at $530-700 \mathrm{~nm}$ is superimposed with the negative purpurin bleach $\left(\mathrm{Bl}_{\mathrm{Pur}}\right)$, with its characteristic features originating from the $\mathrm{Q}_{x}$ and $\mathrm{Q}_{y}$ bands peaking at 583 and $699 \mathrm{~nm}$, respectively (Figure 6.4, green line). Note, that also the features of the purpurin bleach $\left(\mathrm{Bl}_{\mathrm{Pur}}\right)$ appear during the first few tens of femtoseconds, revealing that at least part of the ET process must occur rapidly right after the excitation.

Medium time range. The $\mathrm{S}_{1}-\mathrm{S}_{n} \mathrm{ESA}_{\mathrm{Car}}$ band first narrows and then decays in 


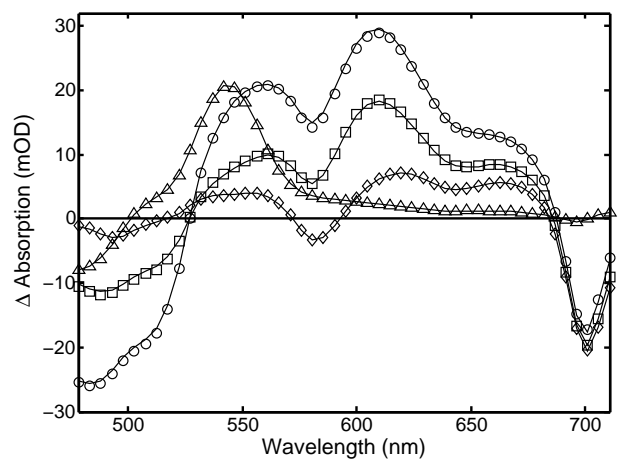

Figure 6.5: Spectral sections of the pump-probe data (symbols) at selected time delays together with the fit (solid line): $420 \mathrm{fs}$ (circles), $7 \mathrm{ps}$ (squares), $50 \mathrm{ps}$ (diamonds), and $6.2 \mathrm{~ns}$ (triangles)

picosecond timescales. This is accompanied by the partial decline of $\mathrm{B}_{\mathrm{Car}}$ (Figure 6.4, red line and cyan lines). Note here, that even after the positive signal due to the ESACar around $550 \mathrm{~nm}$ has disappeared, there clearly is some additional transient state giving rise to the positive signal that lies between 500 and $680 \mathrm{~nm}$ (Figure 6.4, magenta line). The features of the $\mathrm{Bl}_{\mathrm{Pur}}$ remain clear.

Late time range. Here, a positive signal between 520 and $570 \mathrm{~nm}$, belonging to the carotenoid triplet $\mathrm{T}_{1}-\mathrm{T}_{\mathrm{n}} \mathrm{ESA}_{\mathrm{T}}$, rises in nanosecond timescales. At the same time, corresponding growth of $\mathrm{Bl}_{\mathrm{Car}}$ at the blue end of the spectrum and recovery of $\mathrm{Bl}_{\mathrm{Pur}}$ at the red end can be seen.

\subsubsection{Oscillations}

At the pump intensity used in the pump-probe scans $(100 \mathrm{~nJ})$, we also detect subtle signatures of coherent wavepacket oscillations. The oscillations have their maximum amplitude on the red side of the carotenoid ground-state absorption at 520-560 nm, and disappear within a few picoseconds. To further study these oscillations, scans with increasing pump intensities up to $1000 \mathrm{~nJ} /$ pulse were made. The oscillations were analysed by fitting a simple exponential function to the early time data at $546 \mathrm{~nm}$ (Figure 6.7, left panel, red curve). The resulting curve was then subtracted from the data, and FFT was then applied to the residual signal, revealing a Fourier spectrum comprised of four frequencies contributing to the oscillatory pattern, 217, 530, 795, and $1012 \mathrm{~cm}^{-1}$, with periods of $150,63,42$, and $33 \mathrm{fs}$, respectively. The most pronounced peak is at frequency $530 \mathrm{~cm}^{-1}$, whereas the other peaks were discernible only at higher pulse energies. The amplitude of the oscillations increases linearly with the increasing pump energies, as shown in Figure 6.7 (see inset of the right panel). 


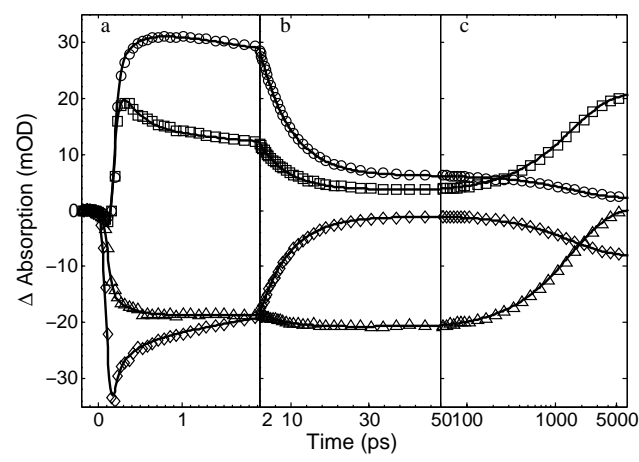

Figure 6.6: Time traces of the pump-probe data (symbols) at selected wavelengths, together with the fit (solid line): $478 \mathrm{~nm}$ (diamonds), $546 \mathrm{~nm}$ (squares), $610 \mathrm{~nm}$ (circles), and $700 \mathrm{~nm}$ (triangles). Note the three different time axes. a: 0-2 ps, linear; b: 2-50 ps, linear; and c: $50 \mathrm{ps}$ to $6.2 \mathrm{~ns}$ (logarithmic).

\subsection{Discussion}

The sequential model served as a first step in the data analysis, giving information on the rate constants and general spectral features of the data. In other words, by resolving and comparing the energy-level diagram of the dyad to the one of $\mathrm{LH} 2$, it is possible to understand how well the dyad mimics the salient features of the kinetics in LH2. In addition to the branching ratio between the functional channel (energy transfer, ET) and the loss channel (internal conversion, IC) several questions remain. Are there additional dark states involved in the kinetics? Do we see evidence of impulsive Raman scattering (IRS), possibly populating the hot ground state $\left(\mathrm{HotS}_{0}\right)$ ? Is it feasible to resolve a clean enough molecular feedback for the learning-loop experiment as used in LH2 coherent-control experiments? What wavelengths and time positions, if any, are suitable to detect signatures of the IC and ET processes?

\subsubsection{Target analysis}

With the previous discussion in mind, the results of a target analysis are discussed here. In this second step of the data analysis, assumptions based on physical intuition and previous knowledge are put to use, some of which were already presented in the Results section, when assigning molecular states to certain spectral features that are depicted by the experimental data and in the EADS. The goal of the target analysis is to find the energy-level diagram, the species-associated spectra (SAS), connections and rate constants, which best describe the measured data. In doing that, one may refer to results previously reported for similar molecules and thus assign certain constraints that are based on information already available.

In principle, target analysis is model testing. Also here, various models, including models with different numbers of states and connections between them, have been 


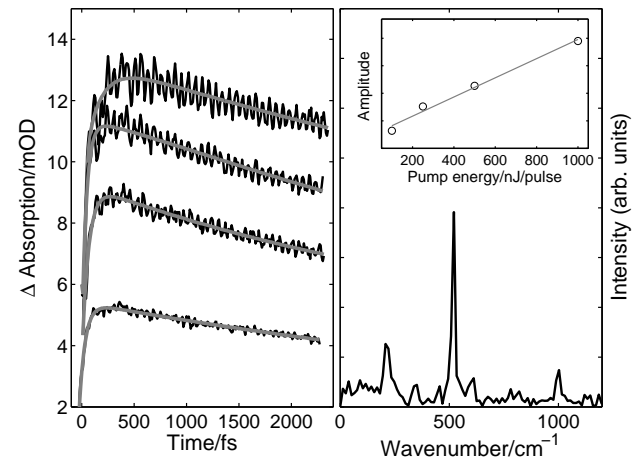

Figure 6.7: Coherent wave packet oscillations. Left panel: pump-probe traces with increasing pump energies $(100,250,500$, and $1000 \mathrm{~nJ} /$ pulse) at $546 \mathrm{~nm}$. Right panel: FFT of the oscillatory pattern at the highest pump energy showing peaks at 217, 530, 795, and $1012 \mathrm{~cm}^{-1}$. Right panel inset: linear pump-energy dependence of the $530 \mathrm{~cm}^{-1}$ peak amplitude.

tested on the dyad pump-probe data. In the following discussion, the results of the analysis are further elaborated, together with a more detailed discussion over previously presented models for the carotenoids and LH2 complex.

The best model is comprised of seven energy levels (i.e., spectral species): the ground states of carotenoid and purpurin (Figure 6.8, brown and black lines, respectively); five carotenoid and one purpurin excited states, carotenoid $\mathrm{S}_{2}, \operatorname{Hot} \mathrm{S}_{0}, \operatorname{Hot}_{1}$, $\mathrm{S}_{1}$, and the triplet (Figure 6.8, blue, purple, red, cyan, and blue lines, respectively); and purpurin $S_{1}$ (Figure 6.8, green line). The resolved time constants are presented in the Table 6.1. For clarity, the discussion proceeds with the help of the three time regimes already introduced in the previous section.

Early time range. The carotenoid $\mathrm{S}_{2}$ and the carotenoid Hot $\mathrm{S}_{0}$ are populated by the laser pulse. Thus, the model includes two states that are initially populated by the pump pulse $\left(S_{2}\right.$ and $\left.\operatorname{Hot}_{0}\right)$. The initially excited population from the $S_{2}$ state decays to the vibrationally $\operatorname{HotS}_{1}$ state by internal conversion with a rate constant of $1 / 110 \mathrm{fs}^{-1}$, from which it further undergoes a cooling process that results in the $S_{1}$ population with a rate constant of $1 / 290 \mathrm{fs}^{-1}$.

A competing pathway branches from the initially excited $\mathrm{S}_{2}$ state toward the purpurin excited states. This carotenoid-purpurin singlet-singlet ET has a rate constant of 1/50 $\mathrm{fs}^{-1}$ and gives an ET/IC branching ratio of 69:31 between ET and IC, which is in good agreement with the ratio determined by the steady-state excitation measurements (ET/IC is 67:33).

The carotenoid $\mathrm{S}_{2}$ excited state has a stimulated emission spectrum located roughly where the carotenoid fluorescence is previously reported to exist [80]. The exact features of the resolved ultrafast spectral band are slightly blurred due to the limited time resolution and a small coherent artefact present in the data.

The vibrational-cooling process of the $S_{1}$ state is described by including an additional Hot $\mathrm{S}_{1}$ level into the model. Accordingly, the algorithm finds a SAS for this 


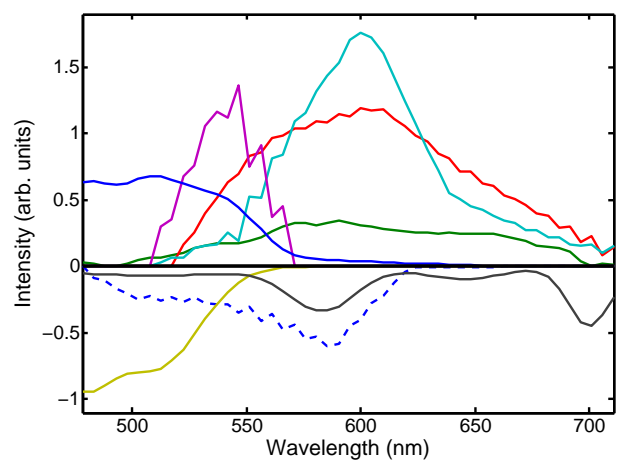

Figure 6.8: Species associated spectra (SAS) obtained by the target analysis employing the energy-flow diagram of in Figure 6.9. The following spectral species are presented for the carotenoid moiety: bleach $\left(\mathrm{Bl}_{\mathrm{Car}}\right), \mathrm{S}_{2} \rightarrow \mathrm{S}_{0}$ stimulated emission $\left(\mathrm{S}_{2}\right), \mathrm{Hot}_{1} \rightarrow \mathrm{S}_{\mathrm{n}}$ excitedstate absorption $\left(\mathrm{Hot}_{1}\right)$, Hot $\mathrm{S}_{0} \rightarrow \mathrm{S}_{2}$ excited-state absorption $\left(\mathrm{HotS}_{0}\right), \mathrm{S}_{1} \rightarrow \mathrm{S}_{\mathrm{n}}$ excited-state absorption $\left(\mathrm{S}_{1}\right)$, and $\mathrm{T}_{1} \rightarrow \mathrm{T}_{\mathrm{n}}$ excited-state absorption $\left(\mathrm{T}_{\mathrm{Car}}\right)$. For the purpurin moiety: bleach $\left(\mathrm{Bl}_{\mathrm{Pur}}\right), \mathrm{S}_{1} \rightarrow S_{\mathrm{n}}$ excited-state absorption (PurQ $\left.\mathrm{y}\right)$.

intermediate state that extends more broadly than the cooled $\mathrm{S}_{1} \mathrm{SAS}$, in consequence describing spectral narrowing. The spectral narrowing of the $S_{1}$ ESA and the corresponding rate constant is congruent with the work by Polli et al [85]. In their study on rhodopin glucoside in benzyl alcohol and in LH2, they found that $\mathrm{S}_{2}$ decays to hot $\mathrm{S}_{1}$ that cools sequentially with two time constants: 50 and $500 \mathrm{fs}$. Polli et al. used $\sim 10$ fs pulses, and likely, the rate constant determined here manifests the lower time resolution used in this study.

Table 6.1: Rate constants of energy-flow pathways

\begin{tabular}{lccccc}
\hline $\begin{array}{l}k_{\mathrm{IC}} \\
\left(\mathrm{fs}^{-1}\right)\end{array}$ & $\begin{array}{c}k_{\mathrm{ET}} \\
\left(\mathrm{fs}^{-1}\right)\end{array}$ & $\begin{array}{c}k_{1} \\
\left(\mathrm{fs}^{-1}\right)\end{array}$ & $\begin{array}{c}k_{2} \\
\left(\mathrm{ps}^{-1}\right)\end{array}$ & $\begin{array}{c}k_{3} \\
\left(\mathrm{ps}^{-1}\right)\end{array}$ & $\begin{array}{c}k_{\mathrm{ISC}}+k_{4} \\
\left(\mathrm{~ns}^{-1}\right)\end{array}$ \\
\hline $1 / 110 \pm 5$ & $1 / 50 \pm 4$ & $1 / 290 \pm 15$ & $1 / 7.8 \pm 0.1$ & $1 / 8.3 \pm 0.1$ & $1 / 1.5 \pm 0.2$
\end{tabular}

It is generally known that the $\mathrm{S}_{1}$ ESA band of carotenoids contains a pronounced shoulder on the blue side of the peak [80]. This spectral feature has recently come into focus by several groups, and various models (including additional dark states [88-90] or alternatively a hot ground state $[95,96]$ ) have been presented for carotenoids in solution as well as for the LH2 complex [93, 97]. The previous study of the dyad by Macpherson et al. preceded this development and hence did not address these aspects of the carotenoid photophysics. Here, the model of Figure 6.9 includes a carotenoid hot ground state $\left(\operatorname{Hot}_{0}\right)$, which is populated instantaneously within the excitation pulse by an impulsive stimulated Raman scattering (ISRS) process [98]. The popu- 
lation of $\mathrm{HotS}_{0}$ by ISRS has been previously reported by Wohlleben et al [99, 100], and by Andersson and Gillbro [95]. Attempts to fit the data with models including an additional dark excited state, lying energetically below the $S_{2}$ state, and being populated from the $S_{2}$ state, were also made. However, the algorithm was unable to find any fit with a comparable $\chi^{2}$ value as was reached by the model of Figure 6.9. In light of these results as well as the previous results from using various ultrafast techniques [101] that claim that the general dynamics of the carotenoids are best explained by a model with a HotS $\mathrm{S}_{0}$ state, we conclude that the carotenoid in the bioinspired dyad system behaves more as a carotenoid in the solvent; no additional excited states were found nor any ultrafast triplet formation via these states is detected as is the case in the LH2 complex [93].

Medium time range. The vibrational cooling from the HotS $S_{1}$ to the $S_{1}$ state is followed by the decay of the carotenoid $S_{1}$ state to $S_{0}$ state by internal conversion with a rate constant of $1 / 7.8 \mathrm{ps}^{-1}$. The hot ground state of the carotenoid decays slightly slower $\left(1 / 8.7 \mathrm{ps}^{-1}\right)$. In this time regime, the purpurin $\mathrm{Q}_{y} \mathrm{ESA}$, present already at early times, remains effectively unchanged.

The $S_{1}$ spectrum has its maximum at $600 \mathrm{~nm}$, and the shape with its long wing extending toward the red is familiar from previous studies on the carotenoid excited state. In natural light-harvesting complexes, both the $S_{2}$ and the $S_{1}$ states act as donors in the ET. In the case of the LH2 complex, it has been shown that the major part of the energy is transferred directly from the initially excited carotenoid $\mathrm{S}_{2}$ state and that only a minor part $(\sim 0.5 \%)$ of the energy is transferred from the carotenoid $\mathrm{S}_{1}$ state to the $\mathrm{B} 800$ molecules [82]. The stepwise $\mathrm{CarS}_{1} \rightarrow \mathrm{B} 800 \rightarrow \mathrm{B} 850$ is seen as a rising bleach signal at $860 \mathrm{~nm}$ in the pump-probe data. Previously, it has been reported that in the dyad, all of the ET occurs directly from the carotenoid $\mathrm{S}_{2}$ state [77]. The rise of the negative bleach signal in LH2 at $860 \mathrm{~nm}$ may originate from the ET from the carotenoid $S_{1}$ state, increasing the amount of excited Bchl molecules, or from the intramolecular internal-conversion process occurring from the carotenoid $S_{1}$ state to the carotenoid ground state. The latter case is naturally possible only if the wing of the positive carotenoid $\mathrm{S}_{1} \rightarrow \mathrm{S}_{n}$ excited-state absorption band extends to overlap with the Bchl bleach, which is not likely the case in LH2. However, in the dyad, the bleach of the purpurin is located some $150 \mathrm{~nm}$ closer to the peak of the $S_{1} E S A$, at $700 \mathrm{~nm}$. Hence, it is impossible to say, as to whether the rising bleach in the dyad originates from ET from the carotenoid $S_{1}$ state, or if it is merely a signature of the decaying wing of the $\mathrm{S}_{1} \rightarrow \mathrm{S}_{n}$ ESA. Therefore, even using sophisticated global analysis techniques, we cannot rule out the possibility of ET from the carotenoid $S_{1}$ state. However, the contribution of this channel would be small: at most only a few percent.

Late time range. After the population in the carotenoid $\mathrm{S}_{1}$ and $\mathrm{HotS}_{0}$ states has decayed, the excess energy left in the system is located in the purpurin $\mathbf{Q}_{y}$ state. The ESA spectrum of the purpurin $\mathrm{Q}_{y}$ state spreads across the spectrum from 500 to $700 \mathrm{~nm}$. From this state, part of the population is relaxed via internal conversion to the ground state, and part of the population undergoes an inter-system crossing (ISC) process from purpurin $\mathrm{Q}_{y}$ to the purpurin triplet state and further a triplet-triplet ET from purpurin to carotenoid $\mathrm{T}_{\mathrm{Pur}} \rightarrow \mathrm{T}_{\mathrm{Car}} \mathrm{ET}$. The carotenoid triplet ESA band arises with a rate constant of $1 / 1.5 \mathrm{~ns}^{-1}$. The ET from the purpurin triplet state to the carotenoid triplet state is fast as compared to the ISC; thus, no population is accumulated 


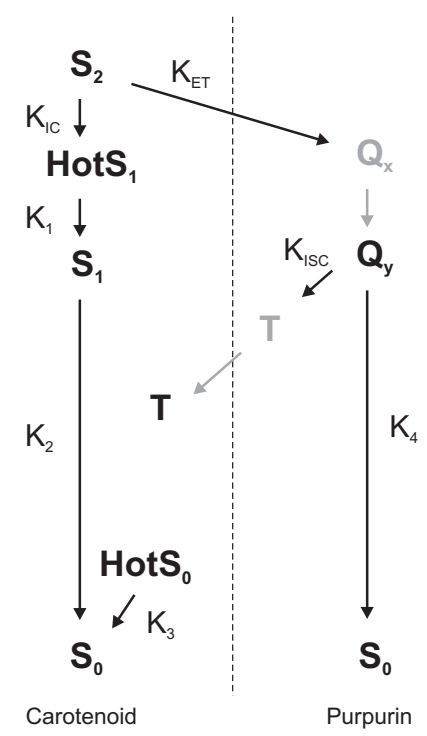

Figure 6.9: Energy-flow diagram of the bioinspired dyad system.

in the purpurin $T_{1}$ state. Consequently, no spectral signature of the purpurin $T_{1}$ state can be resolved from the data.

\subsubsection{Comparison: Natural versus artificial}

The early time dynamics plays the most important role when considering the dyad as a model system to mimic the dynamics of the LH2 complex. The crucial branching occurs from the initially excited $\mathrm{S}_{2}$ state in less than $100 \mathrm{fs}$. In LH2, the ratio is known to be 50:50, where as the dyad shows a 70:30 branching ratio. The rate constants are comparable; in both systems, the internal conversion occurs in little over $100 \mathrm{fs}$. Better efficiency of the ET in the dyad is due to faster ET, even though there is only one chromophore accepting the energy (Table 6.2).

According to the Förster mechanism, the efficiency of the ET depends on the interchromophore distance, the relative orientation of the two chromophores, and the overlap between the absorption and the emission spectra. The absorption of the carotenoids is at the same wavelengths in both systems, as are the $\mathrm{Q}_{x}$ bands of the accepting molecules (purpurin and Bchls). Accordingly, the rapidly decaying $S_{2}$ emission spectra are also likely to exist at the same wavelength in both systems. Hence, the spectral overlap between the donor emission and the acceptor absorption are of the same order in both systems. The edge-to-edge inter-chromophore distances in LH2 are slightly shorter, and the orientation of the dipoles is fixed in the protein matrix. The fact that the dyad depicts faster ET suggests a more favourable average orientation between the chromophores. The involvement of other mechanisms, possibly mediated by the bridge atoms, has also been discussed [77].

The proposed mechanism behind the enhancement of IC in LH2 by shaped laser 
pulses involves the excitation of a specific low-frequency mode by an ISRS process [44]. A multi-pulse laser field is synchronised to a critical vibrational frequency, or an integer multiple of it, whose activation leads to more rapid internal conversion, thus increasing the efficiency of the loss channel. Interestingly, the dyad depicts coherent wave packet oscillations in the spectral region where the Hot $\mathrm{S}_{0}$ is located. Such oscillations have previously been observed in carotenoids and assigned to ground-state wavepacket motion due to the ISRS process within the pump pulse [85, 102]. This is one feasible explanation here as well, and the $\sim 530 \mathrm{~cm}^{-1}$ peak could be assigned to the beating between the $\mathrm{C}-\mathrm{C}$ and the $\mathrm{C}=\mathrm{C}$ stretching modes at 1004 and $1539 \mathrm{~cm}^{-1}$.

Another interpretation could be the involvement of the solvent (toluene) vibrational modes that are somehow coupled to the solute carotenoid. The resolved wavenumbers of the detected three bands agree well with the known toluene FT-IR Raman spectrum, although differing in their relative amplitudes. A similar observation of solvent modes was made by Shimada et al. in a hyper-Raman (HR) scattering experiment on $\beta$-carotene solvated in cyclohexane [103]. As is the case in the present study, a mode attributed to the solvent was only detected in the presence of the solute $\beta$-carotene but was missing in the neat solvent. The authors ascribed this effect to a solvent induced molecular near field, giving rise to an extra HR band. According to their interpretation, the mixing of the carotenoid $S_{2}$ and $S_{1}$ states is influenced by the solvent vibrations, intervening with the HR process. However, HR scattering is a two-photon process, and the mechanism cannot be the same, since the intensity study (see Figure 6.7) clearly reveals that the amplitude of these oscillations depends linearly on the pump intensity, as expected for an ISRS process [104]. Possibly, the mechanism here can be thought to be analogous to surface-enhanced Raman scattering, in that it requires the presence of carotenoid modes and their near-field coupling to the solvent. However, studies varying the concentration and including experiments with deuterated solvents are needed to confirm such a mechanism. We also note that a further influence of the contributing electronic resonance is crucial, since a similar solvent band in cyclohexane as detected in a degenerate four wave mixing (DFWM) experiment [51] was only to be detected under resonant excitation conditions and was missing when the solute $\beta$-carotene was excited non-resonantly.

\subsection{Conclusions}

We present a detailed time-resolved spectroscopic study on the bioinspired dyad molecule, comparing its structure, spectrum, and dynamics to the LH2 complex from $R$. acidophila. Regardless of the structural simplicity, the dyad mimics the major spectral and kinetic features of the LH2 complex well (see Table 6.2). The major difference found in the photophysics is the energy flow in the carotenoid moiety, which is consistent with the photophysics of carotenoids in solution rather than in the protein environment of the LH2 complex. In addition, the newly found involvement of ISRS process in the photophysics of the dyad arouses further interest in using the dyad in mimicking the coherent control results obtained in LH2, where ISRS is the proposed contributing mechanism.

The caroteno-purpurin dyad is an ideal case for a coherent-control study, having a clear branching of the energy flow between two competing pathways leading to two 
different processes: IC and ET. With the aid of a global fitting procedure, the underlying spectral and dynamical contributions of the processes were resolved from the data in detail. On the basis of the analysis, clear signals for the ET and IC can be resolved in a pump-probe experiment. The amount of energy flowing to the ET channel can be probed around $700 \mathrm{~nm}$ and to the IC channel around $610 \mathrm{~nm}$. The sufficient time window for these signals extends from short times to several picoseconds. This provides a reliable and well-characterised feedback signal for future coherent-control experiments, which aim to manipulate the efficiency of these processes. 
Table 6.2: Structural, Spectral, and Dynamical Comparison between LH2 and Bioinspired Dyad

\begin{tabular}{|c|c|c|c|}
\hline & LH2 & & dyad \\
\hline & & Structure & \\
\hline donor & carotenoid & & carotenoid \\
\hline & rhodopin glucoside $(n=11)$ & & $\beta$-carotene derivative ( $n=10+$ benzene ring) \\
\hline acceptor & bacteriochlorophyll & & purpurin \\
\hline linkage & none & & amide bridge \\
\hline neighbours & 9 repeating units in ring structure & & none \\
\hline surrounding & protein & & solvent (toluene) \\
\hline inter-chromophore distance & 3.4 Car-B800 & & 3.7 \\
\hline (edge-to-edge) $(\AA)$ & 3.6 Car-B850 & & \\
\hline & & Spectra (absorption) & \\
\hline donor $(\mathrm{nm})$ & $450-540$ & & $450-540$ \\
\hline acceptor $(\mathrm{nm})$ & $\begin{array}{l}\text { Bchl-B800 } \\
\text { Bchl-B850 }\end{array}$ & $\begin{array}{l}\mathrm{Q}_{y}: 800 ; \mathrm{Q}_{x}: 592 \\
\mathrm{Q}_{y}: 850 ; \mathrm{Q}_{x}: 592\end{array}$ & $\mathrm{Q}_{y}: 699 ; \mathrm{Q}_{x}: 583 ;$ Soret: 439 \\
\hline & & Dynamics & \\
\hline$k \mathrm{~S}_{2} \rightarrow$ acceptor; $\left(\Phi_{\mathrm{ET}}\right)\left(\mathrm{fs}^{-1}\right)$ & $\begin{array}{l}\mathrm{S}_{2} \rightarrow \text { Bchl-B800 } \\
\mathrm{S}_{2} \rightarrow \text { Bchl-B850 }\end{array}$ & $\begin{array}{l}1 / 280 ;(0.2) \\
1 / 185 ;(0.3)\end{array}$ & $1 / 50 ;(0.7)$ \\
\hline$k_{\mathrm{IC}}\left(\mathrm{S}_{2} \rightarrow \mathrm{S}_{1}\right) ;\left(\Phi_{\mathrm{IC}}\right)\left(\mathrm{fs}^{-1}\right)$ & $1 / 120 ;(0.49)$ & & $1 / 110 ;(0.3)$ \\
\hline$k_{\mathrm{IC}}\left(\mathrm{S}_{1} \rightarrow \mathrm{S}_{0}\right) ;\left(\Phi_{\mathrm{IC}}\right)\left(\mathrm{ps}^{-1}\right)$ & $1 / 14.2$ & & $1 / 7.8$ \\
\hline
\end{tabular}




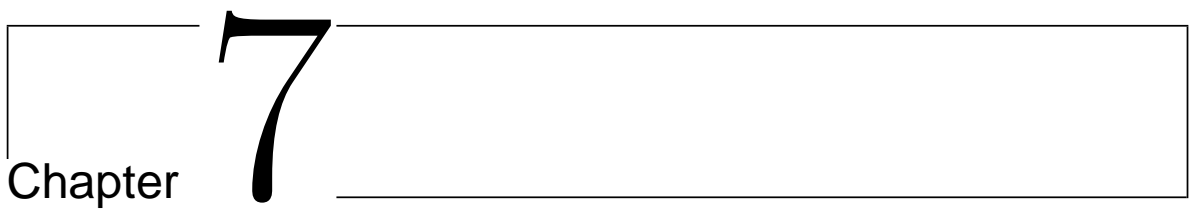

\section{Controlling the efficiency of an artificial light-harvesting complex}

Adaptive femtosecond pulse shaping in an evolutionary learning-loop is applied to a bioinspired dyad molecule that closely mimics the early-time photophysics of the LH2 photosynthetic antenna complex. Control over the branching ratio between the two competing pathways for energy flow - internal conversion (IC) and energy transfer $(\mathrm{ET})$ - is realised. We show for the first time that by pulse shaping it is possible to increase independently the relative yield of both channels, ET and IC. The optimisation results are analysed using Fourier analysis, which gives direct insight to the mechanism featuring quantum interference of a low-frequency mode. The results from the closed-loop experiments are repeatable, robust and demonstrate the power of coherent control experiments as a spectroscopic tool (i.e. quantum control spectroscopy) capable of revealing functionally relevant molecular properties that are hidden from conventional techniques. Finally, open-loop control experiments provide first experimental evidence of the involvement of a hot ground state in the control mechanism. 


\subsection{Introduction}

Artificial photosynthesis is an important challenge of science and technology today. Numerous applications include solar cells and other artificial power sources, lightemitting materials, sensor systems, and other electronic and photonic nanodevices that utilise the conversion of light energy into chemical potentials [18]. Over the last decade, major technological advances have been made using biomimicry, an approach making use of teachings from studies on Nature's wide-ranging selection of highly efficient pigment-protein complexes [105]. It has been shown that integrating lightharvesting antennae with electron-transfer relay systems is a potent way to emulate photosynthesis [19]. Thus, biomimicry has inspired systems based upon complicated natural light-harvesting complexes (LHCs) reduced to their basic elements, and efficient antenna systems based on polymer polyenes covalently attached to tetrapyrroles have been synthesised [106, 107].

The antennae are responsible for the first step of the photosynthesis, capturing energy of the sun and transferring it to subsequent photosynthetic structures where the energy is transformed in chemical potential. Within various natural and synthetic LHCs, blue-green photons are absorbed by carotenoid molecules, from which the energy is transferred to neighbouring porphyrin molecules [80]. This energy transfer (ET) step from the carotenoid donor to the accepting molecular species is the primary process in utilising energy in the window $450-550 \mathrm{~nm}$, and contributes significantly to the functioning of the complex. The efficiency of ET over competing loss processes, such as internal conversion (IC) is a crucial factor in the overall quantum yield of (artificial) photosynthesis. Hence, a high priority is given to understanding the mechanisms of energy flow and mediating processes in order to allow development of more efficient artificial systems.

In this study, we use a novel approach: Adaptive femtosecond pulse shaping in a learning loop $[8,21]$ to control the pathways of energy flow in an artificial LHC. This closed-loop optimisation technique has produced several successful examples in obtaining control over various physicochemical reactions in complex molecules in liquid phase without prior knowledge of the molecular Hamiltonian [21, 108]. Examples extend from control of energy transfer [43], fluorescence yield [109], population transfer [110], selective excitation of vibrational modes [111], and isomerisation reactions [14, 112, 113]. However, in such complex systems the algorithm has to navigate through a multi-dimensional parameter space and the search often results in a complicated, highly modulated pulse shape. This makes the interpretation of the obtained pulse challenging and often leaves the physical mechanism unresolved. Here we show how coherent control techniques can teach us more about the intrinsic properties and interactions of molecular systems, i.e. we perform quantum control spectroscopy (QCS) with pulse shaping [21, 52, 53]. For artificial photosynthesis the goal is to reveal mechanisms and related design criteria that underlie optimal performance for light harvesting.

The system we study is inspired by the LH2 complex from the purple bacterium Rhodopseudomonas acidophila, where many carotenoid and porphyrin pigments are embedded in a ring structure within a protein [79]. Our bioinspired dyad molecule consists of a single donor (carotenoid) and single acceptor (purpurin) moiety, thus the 
structural complexity is reduced significantly. Previously, detailed ultrafast studies revealed that the dyad mimics the salient features of the photophysics of the natural photosynthetic complex [77, 78].

The present study also expands upon previous work where coherent control was used to manipulate the branching ratio between two competing energy-flow pathways in LH2 [43]. In that study the relative efficiency of the loss channel (internal conversion, IC) was improved over the functional pathway (energy transfer, ET) by $30 \%$. The proposed control mechanism [44] involves an excitation of a specific low-frequency C-C-C bending mode enhancing the energy flow to the IC channel. According to this mechanism, a multi-pulse laser field is synchronised to a critical vibrational frequency $\left(\sim 160 \mathrm{~cm}^{-1}\right)$ on the electronic ground state, whose activation leads to more rapid internal conversion, thus increasing the energy flow to the loss channel [44].

To control the pathways of energy flow in the dyad molecule we start blindly, without restricting the optimisation to any particular region of the search space. We then extract recognisable features from the resulting pulse shapes, simplify the parameter space accordingly and test whether a similar result is available using a smaller number of parameters $[10,42]$. This strategy provides a powerful spectroscopic tool that is sensitive to the function of the artificial LHC, thereby revealing important characteristics affecting the efficiency of the light-harvesting process. Furthermore, we show that it is possible to enhance or suppress the functional channel by pulse shapes exploiting different control mechanisms. Ultimately, this approach may lead to the discovery of new design principles to aid the development of more efficient artificial light-harvesting systems.

\subsection{Materials and methods}

The control experiments utilised a closed-loop optimisation strategy [8, 21], whose basic elements are presented in the Figure 7.1a. The optimisation is 'blind' in that it begins with no initial guess, but rather a set of random phases that corresponds to a generation of different pulse shapes. The individual phase patterns are applied to the SLM, which modulates the dispersed spectrum of the femtosecond laser, tuned to the first optically allowed transition of the carotenoid $\left(S_{0} S_{2}\right.$ in Figure $\left.7.1 \mathrm{~b}\right)$ at $510 \mathrm{~nm}$. The resulting pulse shapes are then successively tested on the sample and a feedback signal is derived using transient absorption. Based on this signal, a fitness function is evaluated according to the target of the optimisation, and the individual pulse shapes are ranked. A learning algorithm then selects the best pulse shapes for reproduction and creates a new generation of pulse shapes. Another iteration of the cycle begins, and the loop proceeds to search for pulse shapes that further increase the value of the feedback function, thus closing in on the target objective. A robust calibration method, where each SLM pixel is calibrated and correction for any phase distortion is made by optimising second-harmonic generation in a non-linear crystal, ensured that the shaping introduced no effect on the amplitude of the pump pulse; the fluencies of the excitation pulses were kept moderate, at about $10^{14}-10^{15}$ photons $/ \mathrm{cm}^{2}$ at $510 \mathrm{~nm}$ excitation wavelength.

The target objective is to manipulate the pathways of energy flow in the dyad (Figure 7.1b). Two competing channels, labelled (ET) and (IC), are resolved from the 


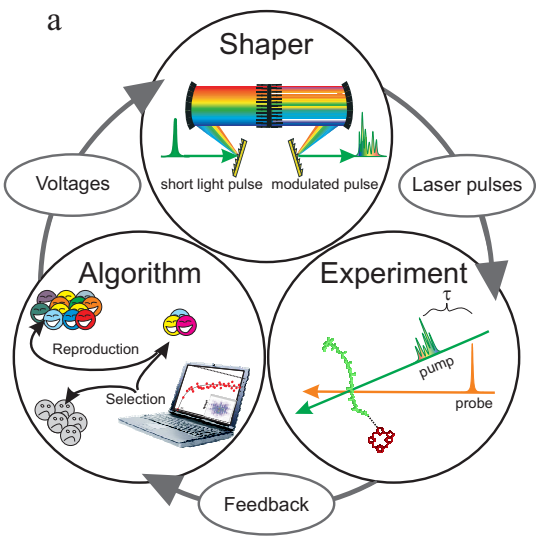

$\mathrm{b}$

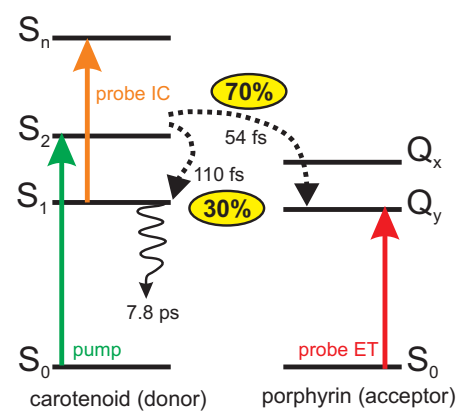

Figure 7.1: Optimisation of the energy-flow in a learning loop. Panel a: Elements of the learning-loop experiment. Panel b: Simplified energy-flow diagram. Following excitation, energy transfer (ET) from the carotenoid $\mathrm{S}_{2}$ state to porphyrin takes place in $54 \mathrm{fs}$. Simultaneously, the first excited state $\left(S_{1}\right)$ of the carotenoid is populated via hot $S_{1}$ state by a competing internal conversion (IC) process in $110 \mathrm{fs}$, resulting in a 30/70 branching ratio between IC and ET. The $\mathrm{S}_{1}$ state decays to the ground state in $7.8 \mathrm{ps}$. The lowest excited energy level of the porphyrin is deactivated on nanosecond timescales.

transient spectrum at 8 ps time delay, and used as feedback for the optimisation. A $10 \mathrm{~nm}$ band cantered at $610 \mathrm{~nm}$ corresponding to the excited-state absorption of the $\mathrm{S}_{1}$ state was used to monitor the loss channel (IC); and a 10-nm band around $700 \mathrm{~nm}$, corresponding to the bleach of the acceptor, for the functional channel (ET). The pump spectrum was cantered at $510 \mathrm{~nm}$ and had a full width of $\sim 45 \mathrm{~nm}$. The algorithm used was a covariance-matrix adaptation (CMA) of the de-randomised evolutionary strategy $[35,94]$.

\subsection{Results}

For the blind optimisation, a large search space described by 208 parameters was chosen in order not to limit the complexity of the pulse shape. The search space was described by a basis having three different frequency ranges (10, 20, and 40 pixels) over the pulse shaper window, and the spectral phase was interpolated between these frequencies. The shaping was done only to the phase and no amplitude shaping was used.

Figure 7.2 shows a learning curve of a blind optimisation in which the target was to maximise the ratio IC/ET, the pathway successfully optimised in LH2 [43]. A total of $15 \%$ increase of the ratio was obtained after 108 generations. The fitness values of the best pulse shapes (red circles) reveal that after an initial jump of about 5\%, the algorithm explores the search space for $\sim 20$ generations before finding a feasible route on the fitness landscape, which gradually results in a further $\sim 10 \%$ increase of the IC/ET ratio, though the optimisation likely has not yet converged. The fitness 


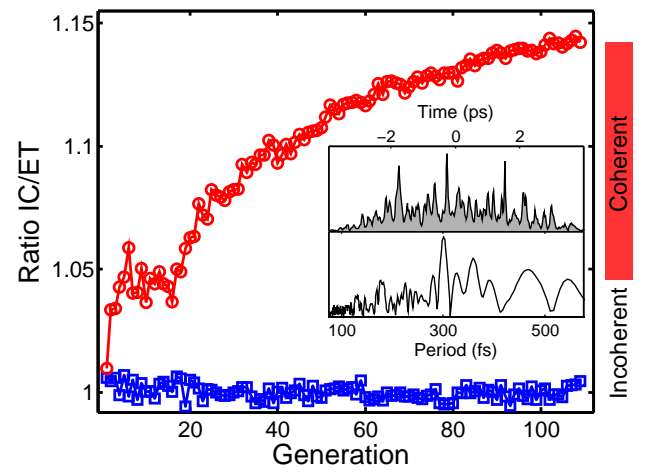

Figure 7.2: A closed-loop optimisation of IC/ET. The learning curve shows an improvement of $\sim 15 \%$ in the fitness value of the best individuals (red dots) compared to the fitness of the TL pulse measured prior to each new generation (blue squares). Inset: cross correlation (upper) and FFT of the cross correlation (lower) of the best pulse of generation 108 .

value of the TL pulse (blue squares) was determined prior to each new generation, providing an excellent indicator that the experimental conditions remained constant during the optimisation.

The optimal pulse shape of the generation 108 (Figure 7.2, inset) spreads as a complicated pulse-train like structure over several picoseconds. The power spectrum of the cross-correlation of the pulse (Figure 7.2, inset) shows a major peak at a period of $\sim 300$ fs corresponding to a frequency within the complex pulse-train structure of $\sim 110 \mathrm{~cm}^{-1}$. The initial jump between the first and the second generation is due to an artefact as will be further discussed in the following section.

According to our strategy, the following step was to move to a more restricted parameter space. Since the first results hinted that pulse trains were a key characteristic, we then used a Fourier-series parameterisation consisting of 20 sinusoidal and 20 cosine functions. In this optimisation the number of parameters was 40 , still sufficiently large to allow for complex pulse shapes. The results of this optimisation are shown in Figure 7.3; now the learning is much faster, such that with just 31 generations a $\sim 10 \%$ increase of the fitness value IC/ET is again found (Panel a). Figure 7.3b shows the optimal phase pattern (blue line) overlapping the pump spectrum (grey area) and Panel $\mathrm{c}$ the corresponding experimental cross-correlation of the resulting optimal control field. The power spectrum of the cross-correlation of the optimal pulse is shown in Figure 7.3d. The figure indicates that the major feature responsible for the increase of the IC/ET ratio is indeed a pulse train with a sub-pulse spacing of $\sim 300 \mathrm{fs}$. This is in agreement with the aforementioned experiment on LH2 , where the optimised pulse shape showed a strong periodic modulation with spacing of $220 \mathrm{fs}$.

We also explored a target objective aimed to improve the relative yield of the energy transfer, using fitness function ET/IC. Figure 7.4a shows an example of a learning curve of an ET/IC optimisation. Initially, the fitness jumps downwards $\sim 8 \%$, but grows to a final improvement of $\sim 13 \%$ higher. Notably, during the learning pro- 

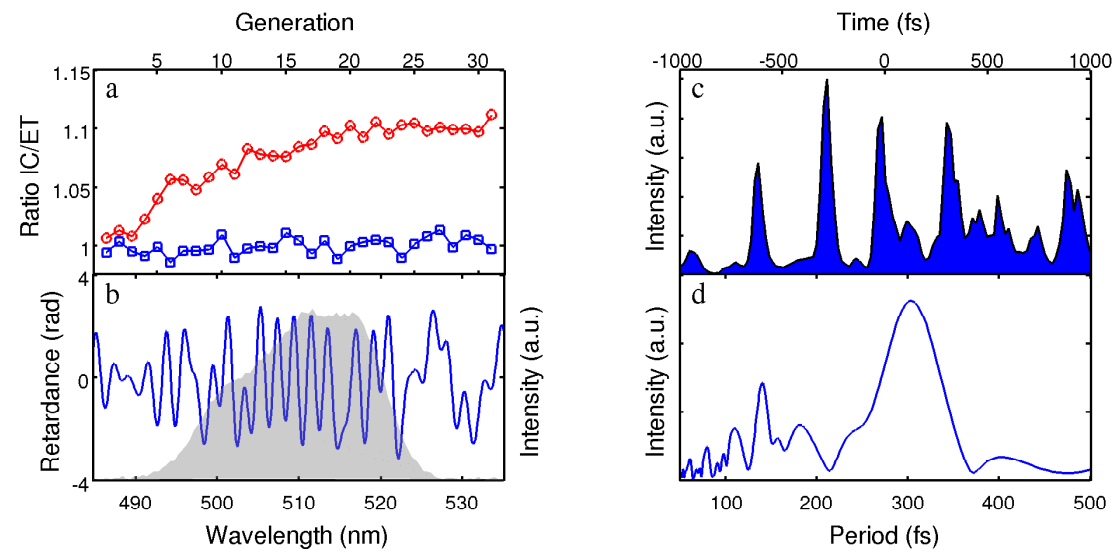

Figure 7.3: Optimisation of the ratio IC/ET using the Fourier-series parameterisation. Panel a: The learning curve shows an improvement of $\sim 10 \%$ in the fitness value of the best individual (red circles). Blue squares indicate the fitness of the TL pulse, measured prior to each new generation. The initial (from TL to the first generation) increase of the fitness value due to the stretching of the pulse is subtracted from the data. Panel b: The optimal phase pattern (blue line) and the pump spectrum (grey area). Panel c: Cross correlation of the optimal pulse shape. Panel d: The power spectrum of the cross correlation.

cess a non-flat phase pattern having an equal fitness value as the TL pulse is found at around the halfway point of the optimisation. However, after the crossing point, more favourable pulse shapes are found that lead to an improvement of the efficiency of the energy transfer process over the competing internal conversion process by $5 \%$, compared to the TL pulse. As in the case of the IC/ET optimisation, the optimal phase pattern, shown in Figure $7.4 \mathrm{~b}$ (blue line), results in a multi-pulse structure shown in Panel c of Figure 7.4. The four-pulse structure has a total duration that is significantly shorter than the best pulse from the IC/ET optimisation, and the most pronounced subpulse spacing is approximately $200 \mathrm{fs}$. The power spectrum of the cross-correlation of the best-found pulse shape in Figure $7.4 \mathrm{~d}$ shows a major peak at a period around $200 \mathrm{fs}$.

\subsection{Discussion}

The experiments show that both product channels (ET and IC) in the artificial LHC are susceptible to coherent control. Using the strategy of sequentially moving from blind optimisations to a restricted parameter space and analysing the optimisations using Fourier analysis, we find that for both product channels a pulse-train structure with varying sup-pulse spacing (around $300 \mathrm{fs}$ for IC and $200 \mathrm{fs}$ for ET) is responsible for the control. The large parameter space gives a lot of freedom for the learning process, but the result is very difficult to interpret. We show that a simpler parameterisation makes optimisations faster while preserving the amplitude of the learning process 

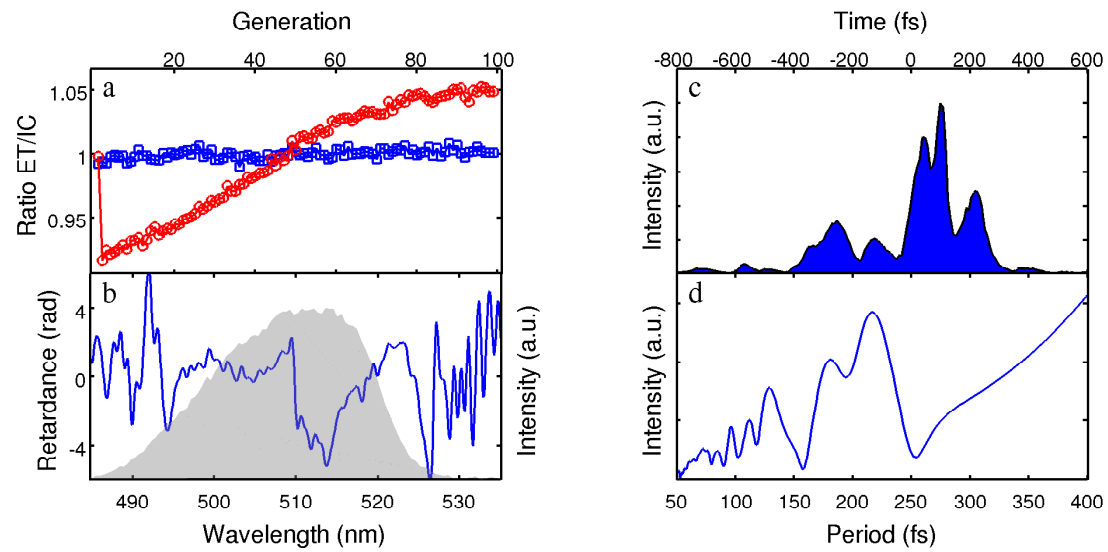

Figure 7.4: The closed-loop optimisation of the ratio ET/IC. Panel a: The learning curve shows an improvement of $\sim 5 \%$ in the fitness value of the best individual (red circles). The blue squares indicate the fitness of the TL pulse, measured prior to each new generation. Panel b: The optimal phase function (blue line) and the pump spectrum (grey area). Panel c: Cross correlation of the optimal pulse shape. Panel d: The power spectrum of the cross correlation.

(10\%). Thus, we have found important directions on the fitness landscape describing a smaller search space still containing the optimal solution.

The initial jump observed in the optimisations is due to a trivial and incoherent control mechanism that simply avoids saturation by pulse stretching, a phenomenon previously discussed for LH2 by Papagiannakis et al. [114]. The effect stems from the fact that the signals for IC and ET have very different life times. Effectively, this means that when the excitation pulse gets longer we observe more signal in IC compared to a transform limited pulse that can readily saturate the carotenoid $S_{0}$ to $S_{2}$ transition. We used the measured saturation curves (data not shown) in combination with the kinetic model of the system [78], and the measured cross-correlations of the pulse shapes along the learning curve to model the effect. The simulations show that compared to the TL pulse the first shaped pulse is invariably stretched in time, resulting in a higher fitness value for IC/ET (or lower in the ET/IC optimisation). This contribution remains constant (fluctuating less than $0.5 \%$ ) over the course of the optimisation, creating a step that underlies the exponential growth curve due to real learning. In addition, we experimentally tested the recorded pulse shapes from the IC/ET optimisation in a repeat measurement using only half the energy. According to the data (not shown) the jump and the 'learning' part have very different intensity dependencies; the initial jump decreased by approximately half of its amplitude whereas the learning part remained the same $(\sim 10 \%)$. We conclude that the initial jump observed in the optimisations is due to a trivial and incoherent control mechanism that simply avoids saturation and that the learning originates from an active control mechanism over the branching of the energy flow in the dyad.

The control mechanism may involve dynamics in an excited and/or the ground 
state of the dyad. Following excitation of the carotenoid moiety to its $\mathrm{S}_{2}$ state, a rapid internal conversion via a conical intersection competes with the energy transfer to the porphyrin [78]. This competition results in a very short lifetime of the $\mathrm{S}_{2}$ state $(<40 \mathrm{fs})$. Considering that the pulse separations in the found pulse shapes are substantially longer, control mechanisms involving wave packet dynamics in the $\mathrm{S}_{2}$ state during the interaction with the pulse can be excluded since population (and thereby also the electronic coherence) decays completely between the sub-pulses. On the other hand, the $\mathrm{S}_{1}$ lifetime of the carotenoid is $7.8 \mathrm{ps}$, and one possibility is that the found pulse shapes are promoting constructive (destructive) interferences between wavepackets that are evolving on the $S_{1}$ potential energy surface. While in some systems vibrational coherence may be preserved during a relaxation between electronic states, previous reports on carotenoids show that passage through the conical intersection between the $S_{2}$ and $S_{1}$ occurs most likely incoherently $[52,102,115]$. The observations in the pump-probe experiments show vibrational coherence only in ground-state potential energy surface, indicating that the vibrational wavepacket created in the $S_{2}$ state does not survive the IC process to the $S_{1}$ potential energy surface [102].

In a recent study on all-trans- $\beta$-carotene in solution, Lustres et al. described a strongly over-damped oscillation between the $S_{2}$ and $S_{1}$ states that causes a recurrence of population in the $S_{2}$ potential energy surface with 300 fs period [116]. Interestingly, this matches the found time separation in the found pulse train in the IC/ET optimisation. Involvement of such a dynamical feature cannot entirely be excluded, but again the lifetime of $\mathrm{S}_{2}$ state suggests that influence of the recurring feature would be very small. Only a minor part of the carotenoid still has excitation, since $70 \%$ flows to the purpurin with ultrafast timescales. Further, it is uncertain whether this recurrence exists in the dyad and with what efficiency.

We now consider a mechanism that incorporates impulsive stimulated Raman scattering (ISRS) of low frequency skeletal modes in the ground state [117]. In the following, we use a pathway approach with potential energy surfaces and wavepackets to describe the multidimensional photophysics of the dyad. Wavepacket generation on specific vibrational modes by shaped pulses [9, 118, 119], which turns into enhancement of vibrational coherence under near-electronic reson ant condition [51, 120, 121], has been demonstrated in various molecules including carotenoids. By periodically modulating the phase of the laser pulse over its spectrum it is possible to prepare wavepackets selectively, and under near-resonant conditions to enhance wavepacket excitation of Raman-active modes. The leading pulses prepare a wavepacket in the vibrationally hot ground state of the carotenoid. By matching the frequency of the pulse train to a ground state vibrational mode, for instance a low frequency twisting of the backbone, we introduce momentum along a trajectory that may take the wavepacket towards Franck-Condon regions not accessed by a Fourier-limited pulse. Subsequently, this leads to an altered evolution on the excited state, either towards or away from the conical intersection between $S_{2}$ and $S_{1}$. In the former case, an excitation of vibrationally hot ground state modes could lead to a more efficient internal conversion process, averting the energy transfer pathway.

Observing the population transfer from $S_{0}$ to $S_{2}$ for the two investigated control scenarios, namely IC/ET or ET/IC optimisations, substantiates this mechanism. 


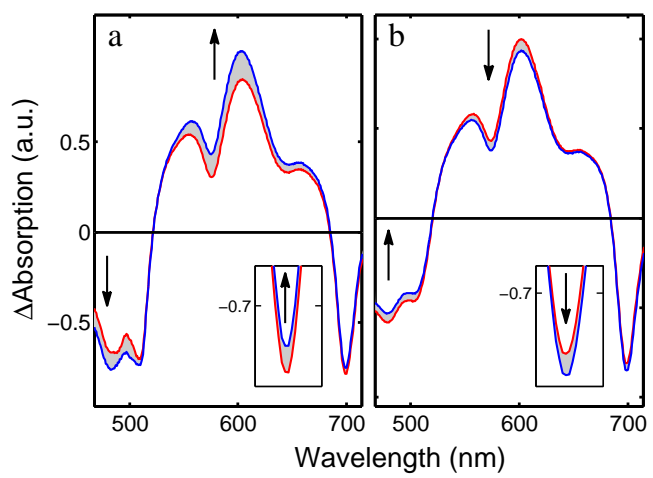

Figure 7.5: The transient spectra at 8 ps probe delay using TL pulse (red line) and the found optimal shapes (blue line). Panel a: IC/ET optimisation. Population transfer increases, IC increases and the ET reduces slightly. Panel b: ET/IC optimisation. Population transfer decreases, IC decreases and the ET increases. Insets: Zoom-in to ET signals at $\sim 700 \mathrm{~nm}$. The arrows indicate the change from the TL to the optimal pulse shapes.

The former case (IC/ET) brings upon an increased population transfer compared to Fourier-limited excitation (see Figure 7.5). The pulse optimal for the ET/IC shaping goal results in a reduction of population transfer (PT) to $S_{2}$ (Figure 7.5b). This can be attributed to a modulation of the population transfer from ground to excited state due to the multipulse interaction [120]. In the case of IC/ET the increased population has momentum along the reaction coordinate leading to wavepacket propagation along a trajectory that brings the wavepacket faster to the conical intersection between $\mathrm{S}_{2}$ and $S_{1}$, hence the improved population transfer almost entirely flows to the $S_{1}$ state. This mechanism is mediated by a pulse-train like structure with a dominant sub pulse spacing of $\sim 300$ fs that matches to a Raman-active mode according to $b=n T_{v i b}$, with $T_{v i b}$ as the vibrational period of the mode, and $n$ as an integer number. The opposite holds for the ET/IC optimisation; the pulse train with $\sim 200 \mathrm{fs}$ pulse separation is out of phase with the multipulse optimal for the other scenario. It avoids the aforementioned build-up of momentum along the low-frequency mode. Such an excitation laser field is more favourable for the ET process by lacking momentum along the trajectory towards the conical intersection. Therefore, through the reduced population transfer we gain a slight increase of energy transfer as illustrated in Figure 7.5. The amount of total population transfer can be estimated from the blue most region of the bleach, where no pump scattering is observed. In the case of IC/ET optimisation PT and IC are increased and ET slightly reduced. In ET/IC optimisation ET is increased and IC and PT decreased.

As a further test of the ISRS mechanism, we monitor how the IC/ET ratio evolves when the parameter $b$ of a periodic phase function $a \sin (2 \pi x / b+c)$, is scanned. In time domain, this so-called $b$ scan renders a pulse train with varying time separation $b$ between the sub pulses. In the $b$ scan, the parameters $a$ and $c$ are kept constant at $\pi$ and 0 , respectively, producing a pulse envelope profile having 11 sub pulses. Figure 7.6a 


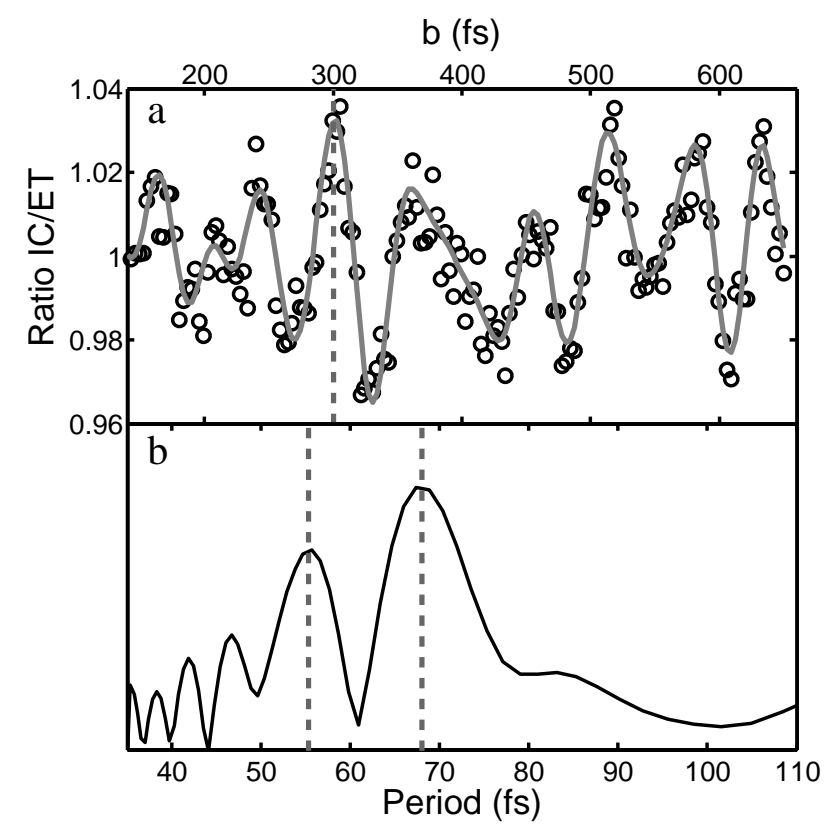

Figure 7.6: Results of the pulse-train scan. Panel a: Ratio IC/ET as a function of the subpulse separation (black circles). To guide the eye, a frequency filtered trace is shown (grey line). Panel b: The power spectrum of the pulse-train scan. The two major contributions are at periods of 55 and $68 \mathrm{fs}$.

shows the result of the $b$ scan after the aforementioned incoherent contribution due to the pulse lengthening has been removed from the data. As expected for the ISRS hypothesis, we observe a maximum at the pulse separation of $300 \mathrm{fs}$. The power spectrum of the $b$-scan trace (Figure 7.6b) reveals two major contributions with periods of 55 and 68 fs. This suggests that the optimal control field found in the closedloop optimisations employs higher harmonics of more than one Raman-active modes, most pronounced having frequencies of $\sim 500 \mathrm{~cm}^{-1}$ and $\sim 600 \mathrm{~cm}^{-1}$. These periods may correspond to low-frequency structural modes as proposed in the work on LH2 $[43,44]$. We note that the control amplitude at $b=300 \mathrm{fs}$ is lower than the $10 \%$ that was found in the closed-loop optimisations. This may be due to the lower pulse energy used in the $b$ scan, reducing the amount of ISRS. Also, the possible interplay between several modes in the control mechanism might be better utilised by the optimal control field found in the closed-loop experiments than the 'clean' and the equidistant pulse train used in the $b$ scan. The underlying physics are likely to incorporate quantum interferences whose manipulation requires more sophisticated pulse shapes than are available using a simple sinusoidal phase function.

To test whether the pulse-train control is sensitive to the relative phases between the subpulses we measured a $c$ scan. In the $c$ scan, the $b$ parameter was fixed to a maximum at $b=300$ fs (see Figure 7.6a) and $c$ was scanned from 0 to $4 \pi$ radians. 


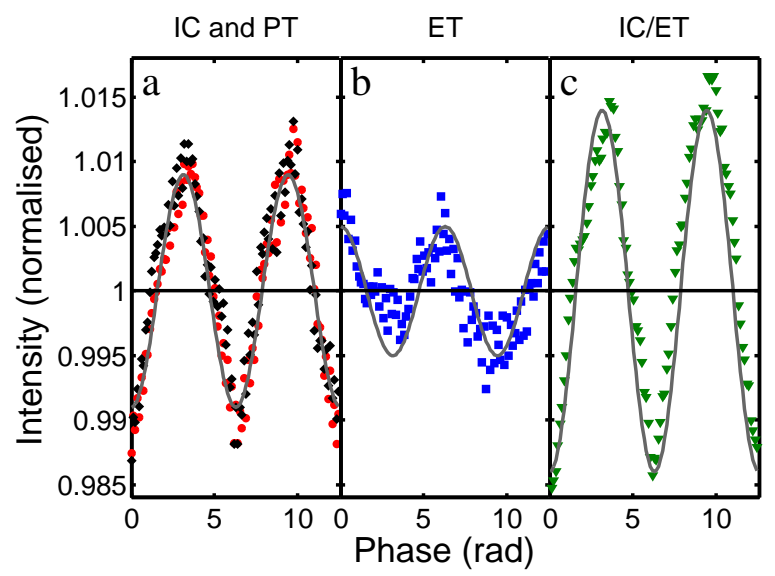

Figure 7.7: Results of the $c$ scan when $b$ is set to the maximum at $b=300$ fs. Panel a: Normalised IC (red circles) and PT (black diamonds) signals. Panel b: Normalised ET (blue squares) signal. Panel c: the resulting IC/ET ratio (green triangles). The grey curves are sinusoidal functions overlayed on data.

As can be clearly seen in Figure 7.7, the signals vary periodically as a function of the parameter $c$. A simple sinusoidal function is overlayed on the data showing and excellent match. The sinusoidal function has a $\pi$ phase shift between the ET and IC channels and the amplitude of the fluctuation is $\sim 2 \%$ for the IC and PT signals and $\sim 1 \%$ for the ET adding up to a $3 \%$ amplitude of the IC/ET ratio. The phase sensitivity of the control suggests that coherence is preserved in the system for at least $300 \mathrm{fs}$.

We also studied the effect of linear chirp to the ET signal. Figure 7.8 shows how ET shows an antisymmetric behaviour. We used a density-matrix approach [120] with three energy levels to simulate the population on the hot ground state. The left panel of Figure 7.8 shows how the diagonal element of density matrix belonging to the hot ground state that describes the population varies as a function of chirp and demonstrate good qualitative correlation with the data. We recognise that such a simple model is an oversimplification of the complex dyad system, however the good correlation with the data indicates that the essential features of the system are described. The results show how the ET yield correlates with the hot ground state population, illustrating the involvement of such a higher-lying ground state in the control mechanism. Hence, the open-loop studies presented here are the first direct experimental proof for the mechanism that was previously proposed for LH2 in reference [44] and earlier in this chapter for the artificial light-harvesting complex.

\subsection{Conclusions}

We have shown how the energy flow in the artificial LHC can be manipulated by femtosecond pulse shaping, to both suppress and for the first time to enhance the 

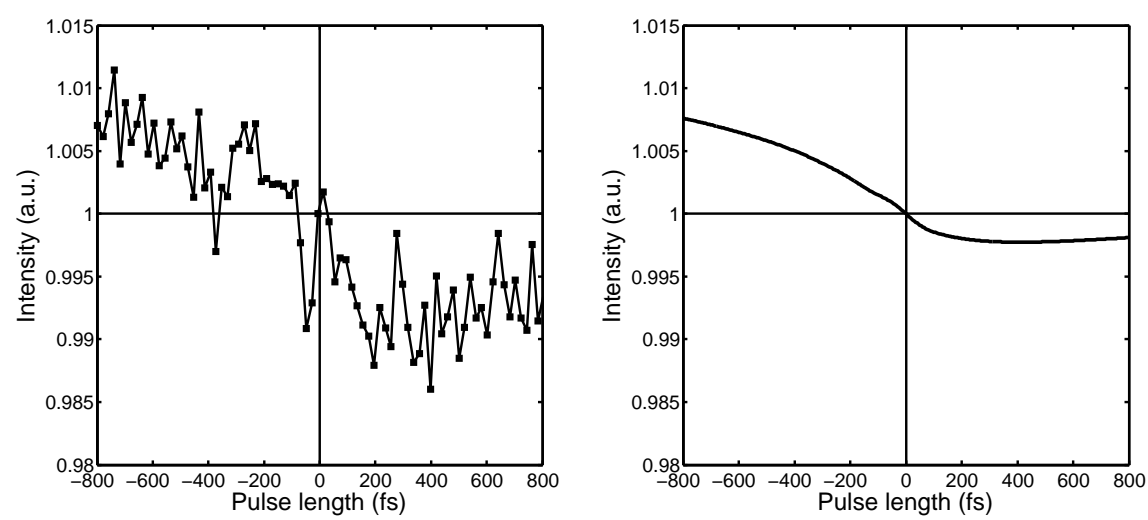

Figure 7.8: Effect of chirp to the ET channel (left) and the simulated hot ground state population.

ET yield. Many repeated runs of the recorded phase shapes from the optimisations were performed indicating that the results are robust. The efficiency of ET in the dyad depends strongly on the photophysics of the carotenoid moiety. By reducing the parameter space in combination with a Fourier analysis of obtained results we were able to track down the functionally important features of this molecular system. A mechanism based on the periodic excitation pulse enhancing the vibrational coherence of low-frequency wavepackets via ISRS process is most likely responsible for the control, analogous to that proposed earlier for the control in LH2 [44]. However, in the dyad the observed effect is smaller, perhaps illustrating the fact that the dyad in solution has more degrees of freedom and possible conformations in the ground and/or excited states [122, 123]. The low-frequency mode that is involved is not restricted by the environment and may gain energy due to intramolecular vibrational redistribution freely, hence leaving the effect of the selective excitation smaller than in LH2 complex, where such a mode is probably more inhibited. In nature, the protein environment/structure seems to aid the ET by posing restrictions to the carotenoid conformational degrees of freedom. This is an important point when considering the design of artificial light-harvesting systems. It appears that coupling to the environment and the restricted conformation and movement have influence on the quantum yield. In future, this will lead to novel design principles for building more efficient artificial solar energy harvesting systems for various applications. 


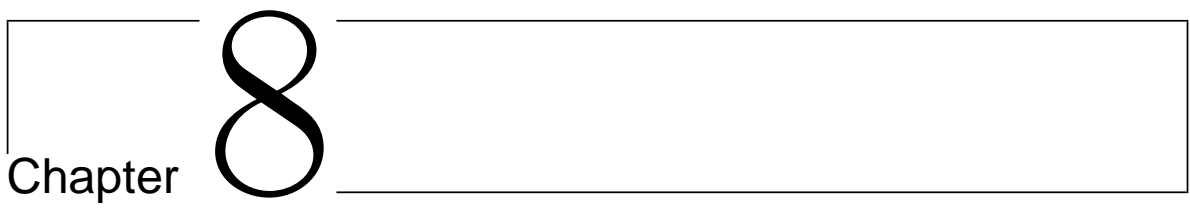

\section{Characterising and controlling photodrug efficiency}

A promising photosensitiser, zinc phthalocyanine, is investigated by means of steadystate and time resolved pump-probe spectroscopies. Spectrally resolved pump-probe data are recorded on time scales ranging from femtoseconds to nanoseconds. Global analysis yields the excited-state absorption spectra and lifetimes, as well as the pathways and efficiencies of the competing relaxation processes from the initially excited $\mathrm{S}_{1}$ state. In addition to the expected nanosecond-scale processes of fluorescence, internal conversion and intersystem crossing that follow the generally accepted kinetic scheme, we also resolve ultrafast dynamics. The nature of these fast processes and their implications to the functional pathway involving triplet formation are discussed.

Optimal control experiemnts show that it is possible to find pulse shapes that manipulate the triplet yield of the model photodrug $\mathrm{ZnPc}$. The robustness of the obtained results is verified by repeated measurements and trivial mechanisms due to saturation are excluded based on careful intensity studies. Further, the performed open-loop control experiments strongly suggest that one possible mechanism influencing the triplet yield involves a sequential resonant two-photon transition to a higher-lying state leading to an increased probability of inter-system crossing to the triplet manifold. 


\subsection{Introduction}

The functionality of photosensitiser molecules arises from their ability to react with molecular oxygen to produce highly-reactive singlet oxygen and other radical species. Among the applications are photodynamic therapy, blood sterilisation and sunlight activated herbicides and insecticides [124, 125]. A good photosensitiser should have a high absorption cross-section at a wavelength suitable for the application, e.g. the optical window of tissue in photodynamic therapy [126]. Singlet oxygen species are created by the interaction between a photosensitiser in its triplet state and oxygen molecule, hence the quantum yield of the triplet state is a key factor when searching for or designing new photosensitisers. Other important factors include the rate of photodegradation, solubility, and the tendency to aggregate [126].

In the end, the efficiency of all these applications depends on the photophysics of the photosensitiser molecule. Improving the photosensitiser to enhance the functional pathway and suppress loss channels is an attractive route to explore by coherent control experiments using shaped ultrafast laser pulses [8]. In this approach, an optimisation experiment is designed with the goal of finding a pulse shape that will improve the yield of a chosen photophysical process in comparison to that obtained with a transform-limited (i.e. unshaped) laser pulse. Improving the triplet yield, and correspondingly the efficiency of the photosensitiser molecule in generating singlet oxygen, is a clear target for an optimisation experiments on photosensitisers. However, prior to these experiments a detailed understanding of photophysical processes involved in the excited state deactivation, as well as their manifestation to the transient spectral signals used as feedback in the control experiments, must be acquired. Here, we report transient absorption experiments and detailed global analysis to extract the pathways, spectral signatures and efficiencies of energy flow in a prototype photosensitiser molecule.

Amongst the most promising second-generation photosensitisers for photodynamic therapy (PDT) are the phthalocyanines [124]. In this study, zinc phthalocyanine $(\mathrm{ZnPc})$ is chosen to serve as a model photosensitiser to study the efficiency of the triplet yield as well as other processes occurring after the initial excitation of the chromophore (see Figure 8.1). ZnPc has been in clinical trials [127, 128], is easy to obtain, stable, produces singlet oxygen with high yields [129], and has a high absorption cross-section in the far-red part of the visible spectrum close to the optical window of tissue. To fully explore the photosensitising qualities of $\mathrm{ZnPc}$, a detailed description of the intramolecular processes, occurring on time scales ranging from femtoseconds to microseconds, is required. We begin by reviewing results from related studies obtained during the last decade, which we will later compare to our own results.

Several experimental studies on the photophysics of $\mathrm{ZnPc}$ and similar compounds (derivatives or different metallophthalocyanines) in solution have been reported [130141], but so far the results fail to depict a coherent picture of the overall photophysics. The generally accepted energy-flow model includes three major intramolecular relaxation pathways from the initially excited $S_{1}$ state. The competition between these processes can be summarised by the modified Gouterman's equation [142]:

$$
\Phi_{\mathrm{F}}+\Phi_{\mathrm{IC}}+\Phi_{\mathrm{ISC}}=1
$$


where $\Phi_{\mathrm{F}}, \Phi_{\mathrm{IC}}$ and $\Phi_{\mathrm{ISC}}$ are the quantum yields of fluorescence, internal conversion and inter-system crossing, respectively.

For the most part, first excited state $\left(S_{1}\right)$ lifetimes (often called fluorescence lifetime $\left(\tau_{\mathrm{F}}\right)$ due to the direct relation between detected fluorescence intensity and $\mathrm{S}_{1}$ population) and fluorescence quantum yields, obtained from fluorescence measurements in different environments, are consistent, and similar values are reported in various studies. Measured $\mathrm{S}_{1}$ lifetimes are in the order of few nanoseconds, for example $\tau_{\mathrm{F}} \sim 2.88 \mathrm{~ns}$ with a quantum yield of $\Phi_{\mathrm{F}} \sim 0.277$ for zinc phthalocyanine tetra sulfonate (ZnPcTS) in DMF [128]. For the sample used in this study ZnPc in DMSO, a $\Phi_{\mathrm{F}}$ of $0.20 \pm 0.03$ is reported by Ogunsipe et al. [140]. Fluorescence yields and decays of the same order have been reported in different environments like proteins, cell suspensions and in vesicles [143, 144], and they are found to be dependent on the surroundings of the chromophore, yet always displaying nanosecond-scale behaviour.

A more complete picture of the nanosecond dynamics is depicted by Bishop et al. in a study of $\mathrm{ZnPc}$ (and its 16-deuterated derivative) in toluene by various photophysical methods [145]. The authors report rate constants of $9.1 \mathrm{~ns}, 38.5 \mathrm{~ns}$, and $5.6 \mathrm{~ns}$, with corresponding quantum yields of $0.34 \pm 0.03,0.08 \pm 0.11$ and $0.58 \pm 0.08$ for $\mathrm{F}$, IC and ISC, respectively. However, as an example of the wide variation in the reported nanosecond dynamics, we note a study by Frackowiak et al. These authors found the quantum yield for the inter-system crossing to be even as high as $0.98 \pm 0.18$ for $\mathrm{ZnPc}$ in air-saturated DMSO [134], using a photothermal measurement technique.

The triplet-state lifetime $\left(\tau_{\mathrm{T}}\right)$ is strongly affected by the presence of molecular oxygen, which drastically reduces $\tau_{\mathrm{T}}$ due to efficient intermolecular energy transfer. Grofscik et al. [131] find a $\tau_{\mathrm{T}}$ of $220 \pm 22 \mathrm{~ns}$ for $\mathrm{ZnPc}$ in air saturated ethanol; Lang et al. [144] report $1.6 \mu \mathrm{s}$ for $\mathrm{ZnPcS}_{3}$ in aerated aqueous protein solution and $205 \mu$ s without the presence of oxygen.

In addition to the nanosecond dynamics described above, also femtosecond and picosecond processes have been reported. The $\mathrm{S}_{1}$ lifetime of $\mathrm{ZnPc}$ in toluene is claimed to be $\sim 35$ ps by Rao and co-workers, a value obtained by degenerate four wave mixing using incoherent light [135]. The setup used, however, was inadequate to quantify components exceeding $100 \mathrm{ps}$. In addition, the authors report a $\sim 3.5$ ps component that is assigned to vibrational relaxation in the $S_{1}$ state, as well as a $S_{n}$ dephasing time of $<170$ fs. Howe and Zhang report an $S_{1}$ lifetime of $\sim 160 \mathrm{ps}$ and an $\mathrm{S}_{2}$ lifetime of $\sim 10$ ps for tetrasulfonated ZnPcS4 in DMSO [132]. The data were obtained by femtosecond pump-probe measurements, where excitation was to the second excited state. In explaining the multi-exponential behaviour of the kinetics authors introduce an energy-flow model that includes an uphill climb from $S_{1}$ to the $S_{2}$ state at room temperature and an inverse saturable absorber model. Recently, in a study of artificial light-harvesting complexes where $\mathrm{ZnPc}$ is covalently linked to a carotenoid molecule, ultra-fast branching of the energy flow in the singlet manifold with time scale of $<100$ fs has been reported by Berera and co-workers [87].

Collectively, the various observations from the ultrafast studies suggest that the conventional energy-flow model (Equation 8.1) fails to depict the entire picture of the kinetics, and that faster processes may also play an important role in the photophysics of $\mathrm{ZnPc}$ and its derivatives. However, the previous results from the ultrafast studies performed on $\mathrm{ZnPc}$ in solution are extremely divergent, and motivate further studies 
in order to resolve the intramolecular dynamics of this system.

Here, we employ pump-probe spectroscopy from femtosecond to nanosecond time scales in order to deliver a more consistent picture of the kinetic processes following the excitation of the photosensitiser. Using a state-of-the-art pump-probe setup and global analysis of the spectrally broad data we focus on the intramolecular dynamics of ZnPc in DMSO, determining the excited state spectra, deactivation pathways, time constants and efficiencies. Besides resolving the nanosecond scale dynamics that follow the conventional energy-level scheme, we show that there indeed are ultrafast processes present. In this context, we also address the role of the solvent, and how solvation dynamics may complicate the analysis of dynamics on ultrafast time scales [146-151]. Global analysis of the temporally and spectrally resolved data allows us to extract a complete picture of the branching ratios between the three competing intramolecular energy-flow pathways as well as the species-associated spectra (SAS). We present an energy-flow model used in the analysis and discuss the origins of all observed dynamics.

\subsection{Experimental}

Steady-state absorbance spectra were measured with a Jasco V-530 spectrophotometer, with the sample in a $1 \mathrm{~mm}$ quartz cuvette (Hellma). For the fluorescence measurements, a Jobin Yvon Spex Fluorolog Tau-3 system was used. The fluorescence spectrum and quantum yield were measured by the ratio method [152] using chlorophyll-a in water as a reference sample. Fluorescence lifetimes were measured in the frequency domain using the modulation technique as described in reference [153]. Fitting software supplied by the manufacturer was used to obtain the fluorescence lifetime.

The pump-probe setup was as in Chapter 6 with minor variations: NOPA produced $\sim 10 \mu \mathrm{J}$ near-transform-limited pulses at $672 \mathrm{~nm}$ with $\sim 30 \mathrm{~nm}$ FWHM and $22 \mathrm{fs}$ pulse duration (pump pulses). The pulse energy was set to $50 \mathrm{~nJ}$ with an adjustable filter, giving a peak power of $3.4 \times 10^{9} \mathrm{~W} \mathrm{~cm}{ }^{2}\left(3.4 \times 10^{14}\right.$ photons $\left.\mathrm{cm}^{-2}\right)$. The sample was circulated in a $1 \mathrm{~mm}$ quartz flow-cell to provide a fresh sample for every pulse to avoid sample degradation or accumulation of long-living states.

The control experiments make use of the same pump-probe setup with a pulse shaper introduced into the pump beam path. In the pulse shaper a liquid-crystal spatial light modulator (SLM; Cambridge Research Instruments) is placed in the Fourier plane of a 4- $f$ zero-dispersion compressor having two 1800 -grooves/mm gratings and cylindrical mirrors $(f=500 \mathrm{~mm})$. A robust calibration method, ensured that the phase and amplitude shaping were uncoupled. The optimisations were performed at a moderate laser fluences.

$\mathrm{ZnPc}$ and DMSO were both purchased from Sigma-Aldrich and used without further purification. The optical density used in the pump-probe experiments was 0.3 at $672 \mathrm{~nm}$ in a $1 \mathrm{~mm}$ path-length flow cuvette, giving a concentration of $\sim 12.6 \mu \mathrm{M}$ (according to $\epsilon=2.38 \times 10^{5} \mathrm{~mol}^{-1} \mathrm{~cm}^{-2}$, reported by Ogunsipe et al. [140]). To check for any sample degradation, the steady-state absorption spectrum was measured before and after the measurements. No changes in OD or spectral shapes were observed, indicating sample stability. All measurements were performed on aerated sample at room temperature. 


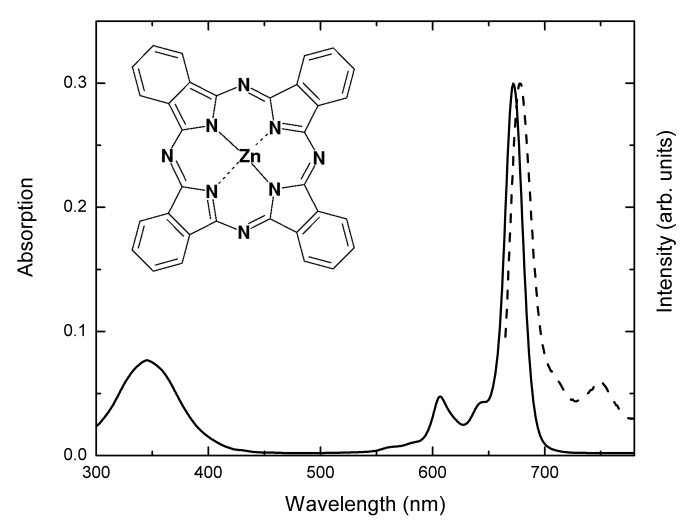

Figure 8.1: Absorption (solid curve) and fluorescence (dashed) of $\mathrm{ZnPc}$ in DMSO. For the latter, the excitation wavelength was $660 \mathrm{~nm}$. Inset: chemical structure of $\mathrm{ZnPc}$.

\subsubsection{Data analysis}

The temporally and spectrally resolved data were analysed globally using software developed in-house, in which the measured 2D-surface is described by a spectrotemporal model. The model comprises of parameters for the initial distribution of population (states (de)populated by the laser), rate constants connecting the states and species associated spectra (SAS) belonging to the states. The ground-state bleach signal is described by inverting the measured absorption spectrum, leaving only its amplitude to evolve as a free parameter. In a similar manner, the stimulated-emission signal was fitted using the inverted fluorescence spectrum that was scaled with a factor of $\nu^{3}$. In the fit this spectrum had its amplitude and spectral position as free parameters. The spectral profiles of all other SAS were described by 50 free parameters, which independently represented spectral amplitudes of every 5th pixel. A spline interpolation was made between these points; hence, no assumption on spectral shapes was made. A small weighting factor, based on the second derivative of the spectra, was used to favour smooth spectral shapes. The fitting makes use of an evolutionary learning loop, where the target objective is to find the physical model that, together with corresponding rate constants, best fits the data. The quality of the fit is evaluated by its $\chi^{2}$ value. The program employs an algorithm based on evolutionary principles. We start with a generation of 20 random sets of parameters, corresponding to 20 simulated surfaces which are each compared to the measured data to determine a $\chi^{2}$ value. The best fitted surfaces are then used to create a new generation of parameter sets. The loop is then let to iterate until convergence to an acceptable $\chi^{2}$ value is reached and the measured data are simulated sufficiently. Details of the fitting program and examples will be presented elsewhere.

For the time-window 0-7 ps we also made use of standard single time-trace fitting at the selected wavelengths. The fine features at the early times have low amplitudes 


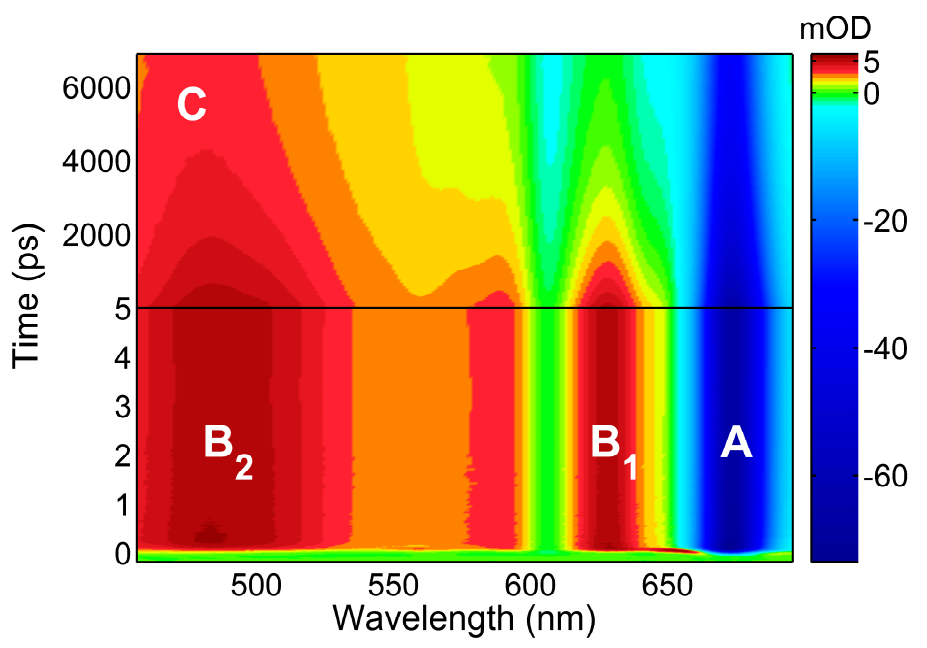

Figure 8.2: Pump-probe data as a function of time (y) and wavelength (x). The characteristic features of stimulated emission and bleach $(\mathrm{A})$, singlet ESA $\left(\mathrm{B}_{1}\right.$ and $\left.\mathrm{B}_{2}\right)$ and triplet ESA $(\mathrm{C})$ are indicated. Note the two different time windows, and non-linear intensity scaling, used to emphasise the ultrafast dynamics.

and the global-analysis program failed to resolve them adequately. In the single trace fitting a sum of exponentials was used as the fitting function. The errors in the resolved time constants (and quantum yields) were determined using the variance in several fits resulting in comparable $\chi^{2}$ values.

\subsection{Characterisation results}

\subsubsection{Steady-state and fluorescence measurements}

The steady-state absorbance spectrum of $\mathrm{ZnPc}$ in DMSO is shown in Figure 8.1. The Q-band $\left(\mathrm{S}_{0} \rightarrow \mathrm{S}_{1}\right.$ ) has a sharp maximum at $672 \mathrm{~nm}$ [154], as well as a vibrational progression to the blue side with a shoulder at $645 \mathrm{~nm}$ and a small peak at $606 \mathrm{~nm}$. The shape of the Q-band spectrum and the positions of the absorption maxima indicate that the $\mathrm{ZnPc}$ sample is in a monomeric form [140]. Further in the ultraviolet, the Soret or B-band can be seen $\left(\lambda_{\max }=345 \mathrm{~nm}\right)$, which we label the $S_{0} \rightarrow S_{2}$ transition.

The fluorescence spectrum (Figure 8.1, $\lambda_{\text {ex }}=660 \mathrm{~nm}$ ) shows an almost perfect mirror image of the absorption spectrum in the Q-band region, including the vibrational progression. The maximum of emission at $680 \mathrm{~nm}$ corresponds to an $8 \mathrm{~nm}$ Stokes shift. The fluorescence quantum yield was determined to be $0.34 \pm 0.2$. Retrieval of the lifetime components, according to the modulation technique used [153], returned only one $3.4 \pm 0.2$ ns component with substantial amplitude. 


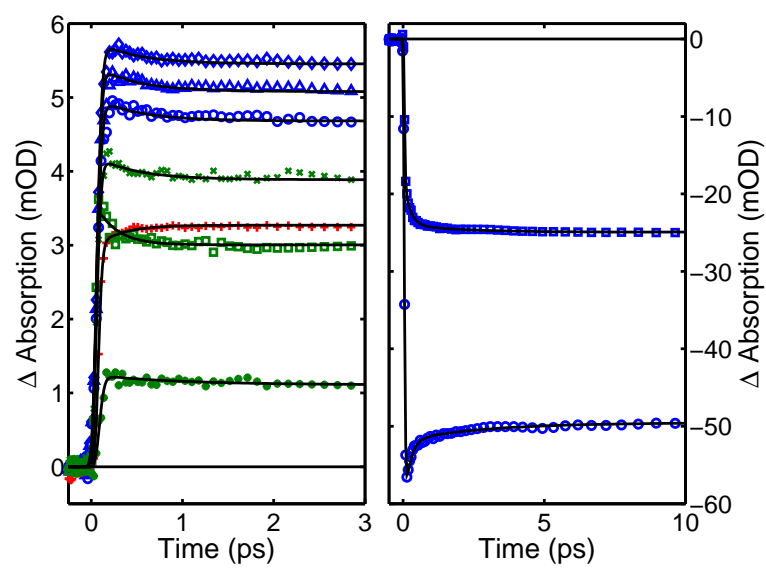

Figure 8.3: Early time transient traces (symbols) and fits (solid curves) at selected wavelengths. Left: 466, 472 and $486 \mathrm{~nm}$ (circles, triangles and diamonds, respectively) in blue; $532 \mathrm{~nm}$ (plus sign) in red; and 612, 620 and $639 \mathrm{~nm}$ (asterisk, crosses, and squares, respectively) in green. Right: 668 (circles) and $686 \mathrm{~nm}$ (squares) in blue.

\subsubsection{Time-resolved spectroscopy}

The 2D-transient surface (Figure 8.2) features broad positive excited-state absorption (ESA) bands, superimposed with a deep negative signal, due to the ground-state bleach and stimulated emission (SE). These signals are identified in Figure 8.2 where they are most pronounced as Bleach $+\mathrm{SE}(\mathrm{A}), \mathrm{S}_{1} \rightarrow \mathrm{S}_{\mathrm{n} 1}$ ESA $\left(\mathrm{B}_{1}\right), \mathrm{S}_{1} \rightarrow \mathrm{S}_{\mathrm{n} 2}$ ESA $\left(\mathrm{B}_{2}\right)$, and $\mathrm{T}_{1} \rightarrow \mathrm{T}_{\mathrm{n}} \operatorname{ESA}(\mathrm{C})$.

Directly upon excitation, an instantaneous bleach is present, as well as other overlapping signals. The negative band originally centred at $672 \mathrm{~nm}$ broadens and the centre of mass moves towards the red. This behaviour is explained by a dynamic Stokes shift: the solvent molecules redistribute in response to the new electron distribution in the excited state of $\mathrm{ZnPc}$, which lowers the energy of this state and correspondingly red shifts the SE signal. These dynamics are more clearly demonstrated in Figure 8.3 (right panel), where transient traces show a rapidly decaying negative signal on the blue side of the band $(668 \mathrm{~nm})$ and rapidly growing negative signal on the red side of the peak of the bleach band $(686 \mathrm{~nm})$. The decay is found to be bi-exponential and is fitted with time constants of $250 \pm 30 \mathrm{fs}$ and $2.5 \pm 0.2$ ps. Further ultrafast dynamics can be resolved in other regions of the transient absorption spectrum (Figure 8.3, left panel). The initial decays of the ESA bands in the spectral regions 460-490 $\mathrm{nm}$ and 615-645 $\mathrm{nm}$ are mono-exponential and can be fitted with a time constant of $450 \pm 50 \mathrm{fs}$, and $250 \pm 30 \mathrm{fs}$, respectively. Likewise, the trace at $532 \mathrm{~nm}$ requires a $250 \pm 30$ fs component, but here with a negative pre-exponential factor (i.e. a rise).

In the later time window extending to nanoseconds, the dominating negative bleach band narrows and the centre of mass moves towards the blue, ultimately reaching per- 


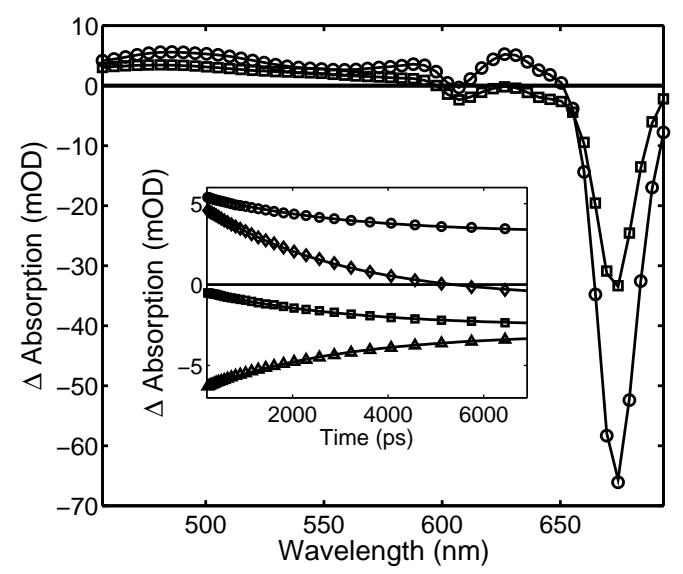

Figure 8.4: Transient spectra (symbols) and the fit (solid curves). Time positions: 7 ps (circles) and $7 \mathrm{~ns}$ (squares). Inset: selected transient time traces and fits (solid curves) at wavelengths $486 \mathrm{~nm}$ (circles), $607 \mathrm{~nm}$ (squares), $631 \mathrm{~nm}$ (diamonds), and $674 \mathrm{~nm}$ (triangles; scaled by 0.1).

fect overlap with the steady-state spectrum $\left(\lambda_{\max }=672 \mathrm{~nm}\right)$. The ESA band at $\sim 630 \mathrm{~nm}$ decays completely, while below $500 \mathrm{~nm}$ the signal persists. Careful inspection of this region shows that maximum, however, is shifted. In Figure 8.4, spectral cross-sections reveal that at 7 ps the ESA band on the blue edge of the spectrum has its maximum at $486 \mathrm{~nm}$, while at $7 \mathrm{~ns}$ the band peaks at $480 \mathrm{~nm}$. Accounting for the effective fluorescence lifetime (the lifetime of the $S_{1}$ state) of $2.9 \pm 0.2 \mathrm{~ns}$, and the fact that ISC process must occur on the same time scales to allow for a significant triplet quantum yield, we assign the band in the early time window to the $S_{1} \rightarrow S_{n}$ ESA and the band at later times to the $\mathrm{T}_{1} \rightarrow \mathrm{T}_{\mathrm{n}}$ ESA. Time evolution in this time window across the measured spectrum is shown by time traces at selected wavelengths (Figure 8.4, inset).

Table 8.1: Lifetimes, and quantum yields

\begin{tabular}{lccc}
\hline & F & IC & ISC \\
\hline QY & $0.28^{a}$ & $0.10 \pm 0.02$ & $0.64 \pm 0.02$ \\
Life time (ns) & $10.1 \pm 0.3$ & $38 \pm 1$ & $4.5 \pm 0.2$ \\
\hline${ }^{a)}$ from reference [155] & & &
\end{tabular}

The late time window of the 2D surface (from 7 ps to $7 \mathrm{~ns}$ ) can be well fitted according the kinetic model corresponding to equation 8.1, in which three competing processes (F, IC and ISC) account for the deactivation of the $S_{1}$ state. The resulting SAS are shown in Figure 8.5 and quantum yields and lifetimes are given in Table 8.1. Note that in the global fitting, the depopulation of $S_{1}$ is described by two pathways 


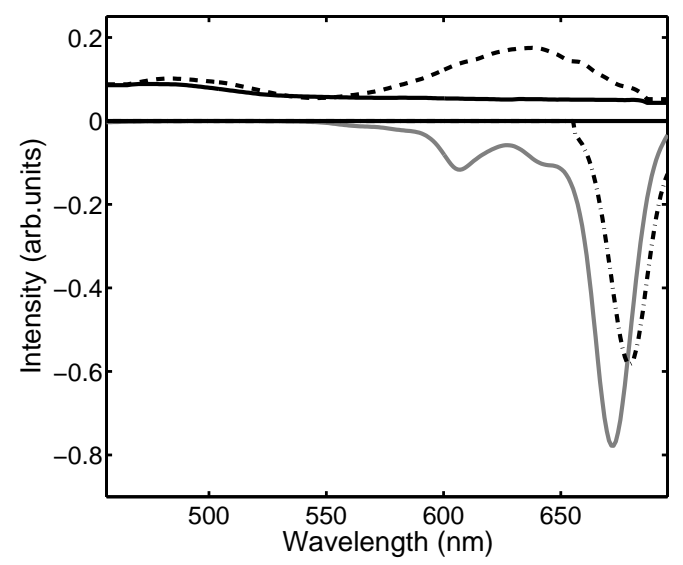

Figure 8.5: Resolved Species-Associated Spectra (SAS) from the late time fitting using the model of Figure 8.1. $\mathrm{S}_{1}$ (solid), $\mathrm{T}_{1}$ (dashed), Bleach (solid, grey), and SE (dash-dotted). For comparison see the original combined transient spectra at 7 ps and 7 ns in Figure 8.4.

with rate constants KISC and $(\mathrm{KIC}+\mathrm{KF})$. Hence, in order to resolve all three quantum yields we make use of equation 8.1, the separately-determined fluorescence quantum yield [155], and the following three equations:

$$
\begin{gathered}
k_{\mathrm{F}}=\frac{\Phi_{\mathrm{F}}}{\tau_{\mathrm{F}}} \\
k_{\mathrm{ISC}}=\frac{\Phi_{\mathrm{T}}}{\tau_{\mathrm{F}}} \\
k_{\mathrm{IC}}=\frac{1-\left(\Phi_{\mathrm{F}}+\Phi_{\mathrm{F}}\right)}{\tau_{\mathrm{F}}},
\end{gathered}
$$

where $k_{\mathrm{F}}$ is the measured fluorescence rate constant and $k_{\mathrm{ISC}}$ and $k_{\mathrm{IC}}$ are the rate constants of inter-system crossing and internal conversion, respectively.

From the SAS (Figure 8.5) it is clear that the ESA bands are very broad and also overlap the bleach/SE signals. The ESA in the singlet manifold shows two distinct bands peaking around $490 \mathrm{~nm}$ and $640 \mathrm{~nm}$, whereas the triplet ESA has only one clear maximum at about $480 \mathrm{~nm}$. This difference explains the shift of the maximum of the ESA signal in the blue region of the measured spectrum, which is due to the decay of population in the $S_{1}$ state, concurrent with the population growth in the $T_{1}$ state. The 2.8 ns lifetime of the $S_{1}$ state determined from the fit matches well with previous studies [128, 137, 155], and the effective fluorescence lifetime measured in this study. The position found for the SE spectrum is red shifted by $8 \mathrm{~nm}$, with respect to the peak of the bleach $(672 \mathrm{~nm})$, congruent with the Stokes shift found in the steady-state fluorescence measurements. Furthermore, the strong negative signal is first broadened on the red side due to $\mathrm{SE}$ and then narrows to the width given by the pure bleach signal when $S_{1}$ state is fully depleted. 


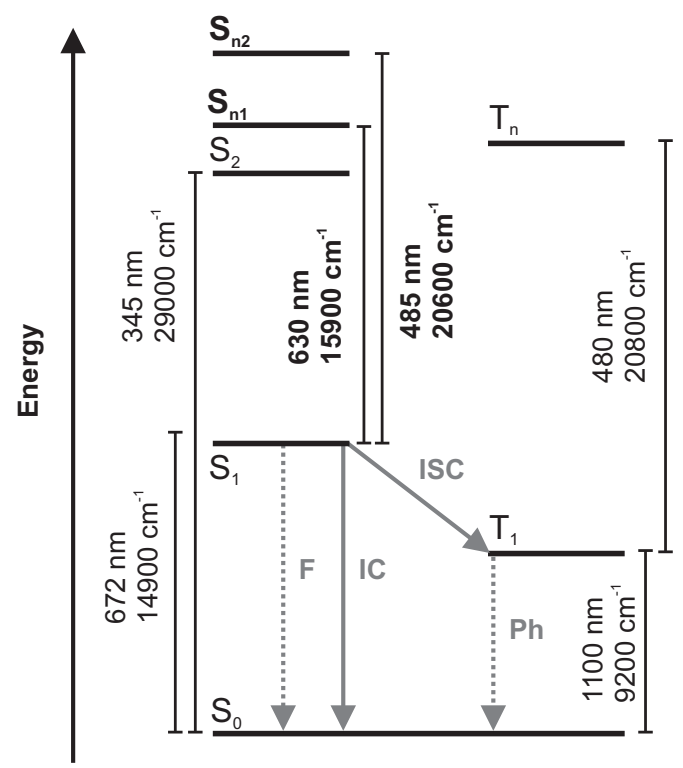

Figure 8.6: Schematic of the proposed new energy-level diagram for $\mathrm{ZnPc}$ showing both the singlet $(\mathrm{S})$ and triplet $(\mathrm{T})$ manifolds and the deactivation pathways from the $\mathrm{S}_{1}$ state: fluorescence, F; internal conversion, IC; inter-system crossing, ISC; and phosphorescence, Ph. Newly found states and energy spacings are shown in bold.

The ultra-fast dynamics observed in the first few picoseconds depict additional processes that cannot be accounted for with such a simple kinetic scheme. Hence, accurate modelling requires a more elaborate description. Bearing in mind that the measured data is a superposition of overlapping signals and that the bleach signal occurs instantaneously, the ultra-fast evolution of the transient 2D-surface must be due to excited-state processes, such as energy flow between electronic states, vibrational wavepacket dynamics and/or solvation processes. To better interpret these fast components we refined the kinetic model, as discussed in the following section.

\subsection{Discussion}

Based on our global analysis of the data, we aim to update the energy-level diagram of $\mathrm{ZnPc}$. As a caveat, we note that the excited-state absorption bands are very broad and the density of states is high; hence, such a simplistic level scheme is likely inadequate in describing the real molecule in solution. This disclaimer is also important in the assignment of energies to the excited states, which are taken from the maxima of each broad transition. Nevertheless, such a model is a good basis for discussion and is useful in providing organised information, once its limitations are recognised.

The ESA signal from the $S_{1}$ state allows us to identify two distinct higher-lying states in the singlet manifold $\left(\mathrm{S}_{\mathrm{n} 1}\right.$ and $\mathrm{S}_{\mathrm{n} 2}$, see Figure 8.6). $\mathrm{S}_{\mathrm{n} 2}$ is accessed from the $S_{1}$ state with probe wavelengths in the range of $460-490 \mathrm{~nm}$, and $S_{n 1}$ in the range 
615-645 nm. Taking the centre of these ESA bands, we put the energies of these levels at 35500 and $30800 \mathrm{~cm}^{-1}$ from the ground state. In addition, the observed ultrafast components indicate the presence of short-lived processes involved in the early-time dynamics of the system. To our knowledge, these processes have not been previously reported for ZnPc. However, other molecules in DMSO have shown similar dynamics attributed to solvation processes [151].

It is well known that solvation dynamics can occur on several time scales [146151]. For example, dielectric relaxation (i.e. the Stokes shift) generally takes place in picoseconds, while inertial components of the solvent dynamics occur on femtosecond time scales. Hence, it is no surprise that these different time scales also show up here. The solvation process is nonexponential in nature, and can be characterised only approximately by a set of exponential decay times. In a study of the solvation dynamics of coumarin 153 in DMSO [151], the spectral solvation response function could be fitted with time constants of $214 \mathrm{fs}, 2.29$ ps and 10.7 ps. The fastest component was assigned to the inertial component of solvation followed by the dielectric nature of the solvation processes. In light of this, we are inclined to attribute the $250 \mathrm{fs}$ and the 2.5 ps components to inertial and dielectric solvation dynamics of the DMSO solvent. The 450 fs time constant, while not directly corresponding to one of the previously determined time constants for DMSO solvation dynamics [151], may also have the same origin. Alternatively, it could reflect intramolecular dynamics occurring in the $\mathrm{ZnPc}$ deactivation, such as relaxation or equilibration between energy levels. Likely targets would be the two degenerate $\mathrm{Q}$ states in $\mathrm{ZnPc}$, which are spectroscopically indistinguishable upon excitation, or an independent precursor energy level that rapidly decays to the $S_{1}$ state. Even though the ultrafast components can be clearly observed in the data over a wide spectral range (see Figure 8.3), the fitting program was unable to extract neither unique spectral bands nor connections to other states for these features without substantial initial input and fitting restrictions. Unfortunately, the data offer no decisive evidence for assigning the $450 \mathrm{fs}$ component; nonetheless, the existence of ultrafast dynamics that cannot be explained by the generally accepted energy-flow scheme is evident, and furthermore is not without precedent.

Previous studies fail to paint a clear picture of the ultrafast dynamics of ZnPc. Bearing in mind that some of these studies were on $\mathrm{ZnPc}$ derivatives (i.e. tetrasulfonated $\mathrm{ZnPc}$ ), we nonetheless feel a comparison is worthwhile. Our justification is based on ab initio calculations that indicate the primary photophysics originate from the central conjugated ring structure of the $\mathrm{ZnPc}$ molecule $[156,157]$.

Howe et al. [132] have suggested a model including an energy-flow equilibrium between $S_{1}$ and $S_{2}$ states in tetrasulfonated $\mathrm{ZnPc}$. At room temperature, $k T$ is approximately $200 \mathrm{~cm}^{-1}$ where as the energy separation between the $S_{1}$ and $S_{2}$ states is $14100 \mathrm{~cm}^{-1}$. Hence, we find it unlikely that a molecule in the $S_{1}$ state could gain enough energy to jump to the $S_{2}$ state, or that there could ever be an equilibrium between the two excited states. Furthermore, the 10 ps lifetime for the $S_{2} \rightarrow S_{1}$ internal conversion seems rather long, especially when comparing to the work by Fournier et al. [136] where a lifetime of $<210$ fs is reported for the same transition in NiPc and in CuPc. Also problematic here is that the data by Howe and co-workers shows a 160 ps component, which is assigned to the decay from $S_{1}$ to the ground state. We find no evidence of this component in our measurements and considering that the 
fluorescence lifetime is in the order of $3 \mathrm{~ns}$ it should simply not be possible to have such a fast decay pathway from the $\mathrm{S}_{1}$ state.

Using degenerate four-wave mixing with incoherent light, Rao et al. found three different time components [135], which were assigned to phase relaxation of $S_{n}$ states $(<170 \mathrm{fs})$, vibrational relaxation $(\sim 3-5 \mathrm{ps})$, and population relaxation from the $S_{1}$ to the ground state (35 ps). Again, such a fast relaxation from $S_{1}$ is unrealistic, considering the known fluorescence quantum yield and lifetime. However, the 3-5 ps found for the vibrational relaxation is of the same order as was found in the present study. Their assignment to a solvation processes is consistent with our resolution of the dynamic Stokes shift.

Berera and co-workers studied ZnPc photophysics in an artificial light-harvesting complex where excitation energy is transferred from the $\mathrm{ZnPc}$ to a carotenoid moiety [87]. To explain the ultrafast dynamics observed in this dyad system, they suggest a model that includes branching from an initially excited precursor state to two different $\mathrm{S}_{1}$ states. The origin for the proposed deactivation scheme is attributed to ground-state heterogeneity. Only one of the $S_{1}$ states connects to the triplet state, while the other channel leads to energy transfer to the carotenoid molecule.

The discrepancies in the measured fluorescence lifetimes and quantum yields can likely be explained by sample concentration effects. In this work, the lifetime determined in the fluorescence measurements (3.4 ns) is somewhat longer than was found in the pump-probe measurements ( $2.9 \mathrm{~ns})$, which is most likely due to the higher concentration used in the pump-probe experiments, leading to self-quenching of the $S_{1}$ population and re-absorption of emitted photons. These phenomena are frequently seen in fluorescent molecules with relatively small Stokes shifts and high absorption cross-sections [153]. The lifetimes and quantum yields reported in the literature vary from $2.88 \mathrm{~ns}$ to $3.4 \mathrm{~ns}$, and from 0.18 to 0.34 , respectively [128, 134, 139, 145]. The cause of this variation likely also stems from the different concentrations and solvents used. In calculating the values for Table 8.1 we chose to use the fluorescence quantum yield of 0.28 [155], corresponding to the lifetime of $2.88 \mathrm{~ns}$ found in this study.

Perhaps the most important photophysical parameter is the quantum yield for triplet formation, which is directly related to the functional photosensitising applications. The found quantum yield of $0.64 \pm 0.02$ for the inter-system crossing is only slightly higher than reported by Bishop et al. (0.58 \pm 0.02$)$ [145], which might be due to the different solvent used. In contrast, a quantum yield close to 1 as reported by Frackowiak et al. seem unrealistically high [134]. It is important to note that the ambiguity of the fluorescence quantum yield discussed above does not affect our determination of the quantum yield of triplet formation, which is resolved directly from the fit of the data with the kinetic model. Hence, the fluorescence quantum yield is only used to calculate the quantum yield of internal conversion process.

Finally, the possible existence of ultra-fast intramolecular dynamics and branching in the deactivation of the $\mathrm{ZnPc}$ photosensitiser molecule opens new opportunities for coherent-control experiments $[158,159]$. In further studies, we will explore the possibility to find a laser pulse shape that leads to enhancement of the functional channel in which excitation energy flows to the triplet state. Success would further imply that it is possible to enhance the efficiency of this photosensitiser by means of pulse shaping. 

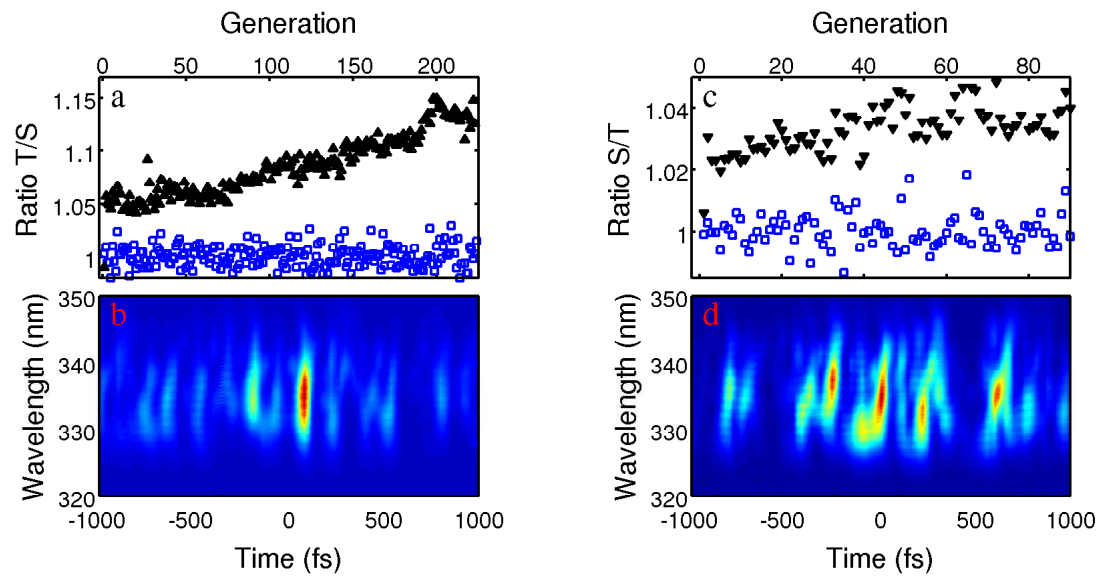

Figure 8.7: Optimal control results on the triplet yield of the model photosensitiser ZnPc. Panel a: The obtained learning curve (red circles) for the ratio T/S and fitness of the TL pulse measured before each generation (blue squares). Panel b: The X-FROG surface of the best pulse. Panel c: The obtained learning curve (red circles) for the ratio S/T and fitness of the TL pulse measured before each generation (blue squares). Panel d: The X-FROG surface of the best pulse.

\subsection{Coherent control results and discussion}

Optimal control experiments

In the optimal control experiments, the singlet (S) signal was obtained by integrating a $10 \mathrm{~nm}$ band from the transient spectrum at $20 \mathrm{ps}$ time delay at the maximum of the found $\mathrm{S}_{1}$ ESA band centred at $490 \mathrm{~nm}$. The feedback signal for the triplet $(\mathrm{T})$ state was measured at $25 \mathrm{~ns}$ using an extra photodiode and additional NOPA tuned to the maximum of the triplet ESA at $480 \mathrm{~nm}$ with a bandwidth of $15 \mathrm{~nm}$. The fitness function was $f=\mathrm{T} / \mathrm{S}$ and $f=\mathrm{S} / \mathrm{T}$ of the triplet yield and singlet yield optimisations, respectively. We used this pump-probe-probe arrangement and fitness functions to aid the algorithm to focus on finding solutions that manipulate the branching ratio between $\mathrm{T}$ and $\mathrm{S}$ rather than solutions that vary the extent of the initial population transfer from the ground state. The initial pulse energies were kept moderate at $500 \mathrm{~nJ} / \mathrm{pulse}$. We chose a large search space having 250 pixels where the phase and amplitude were described independently by 500 parameters. Hence, in these particular optimisations we also used amplitude shaping of the pump spectrum as discussed in the following.

Figure 8.7 shows learning curves from such optimal control experiments together with the X-FROG traces of the resulting best pulse shapes. Panels a and c of Figure 8.7 show that it is possible to manipulate the triplet yield by increasing it some $10 \%$ and decreasing it a few percent with respect to the singlet channel. As is often the case in closed-loop experiments on real molecules in condensed phase, the found pulse shapes show complicated structures that are difficult to interpret directly (Panels b and d). In this particular study we moved directly to open-loop control experiments, rather 

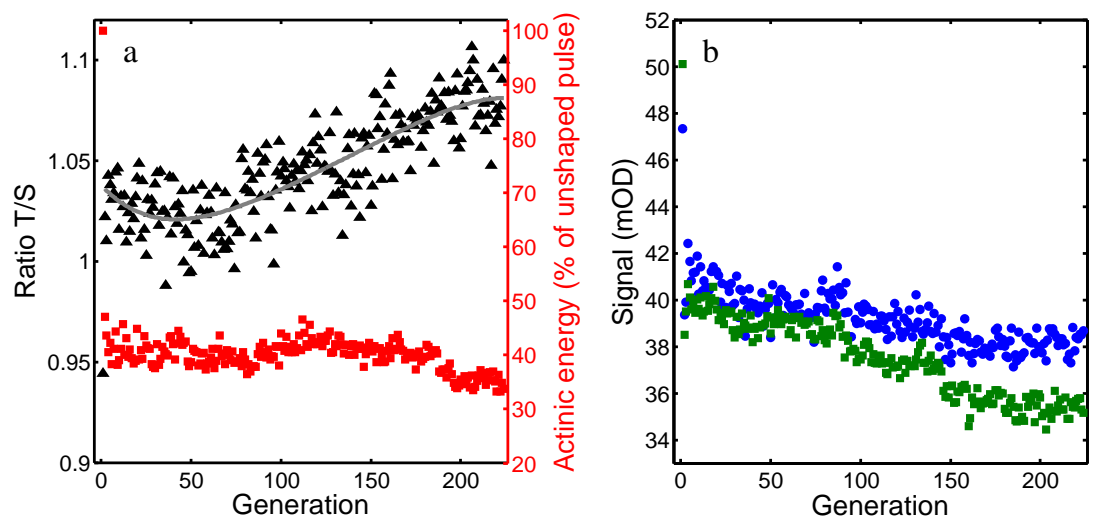

Figure 8.8: Repeated measurement of the T/S optimisation. Panel a: The ratio T/S (black triangles) and normalised actinic energy (red squares). The grey line is a polynomial fit to guide the eye. Panel b: The triplet signal (blue circles) and the singlet signal (green squares).

than performing more elaborated pulse analysis and further optimisations.

To verify the robustness and to further understand the obtained optimal control results we conducted repeated measurements where the fitnesses of the found best pulse shapes were remeasured. The black triangles in Panel a of Figure 8.8 show how the optimisation indeed is robust and repeatable. However, the final and somewhat sharp increase in the fitness around the generation 200 in Figure 8.7 is missing from the repeated results, which implies that some experimental parameter(s) had changed during the last part of the original optimisation, which then resulted in an experimental artifact that is not repeatable and thus not an interesting part of the found control.

The red squares in Panel $b$ of Figure 8.8 indicate the so-called actinic energy of the best individual pulses along the optimisation. Actinic energy is simply an integrated sum of the pump spectrum, amplitude mask, and the absorption spectrum of the ZnPc. The shown values are normalised to 100 for the flat amplitude mask with no amplitude shaping. The actinic energy first drops to $\sim 47 \%$ of the initial value, which is expected for the random amplitude mask of the first generation. From there on the actinic energy stays fairly constant varying in total some $13 \%$. It is interesting to compare how the actinic energy relates to the fitnesses. The initial fall of $\sim 53 \%$ in the actinic energy results in an increase of the T/S ratio of $\sim 6 \%$. Later, along the optimisation such correlations cannot be found. For instance, from generation 50 to 100 the T/S ratio raises with the slightly increasing actinic energy, whereas between generation 150 and 200 the opposite holds: increasing fitness with decreasing actinic energy. In conclusion, the data show that the control involves other features than trivial solutions related to the pulse intensity.

Panel $\mathrm{b}$ of Figure 8.8 shows how the individual signals belonging to $\mathrm{T}$ (blue circles) and S (green squares) behave during the repeated measurement shown in Panel a. The overall population transfer decreases as both of the channels show decreasing sig- 


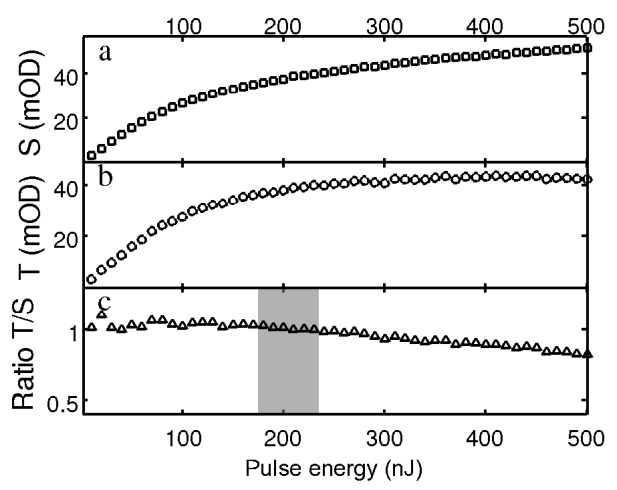

Figure 8.9: Intensity dependence of the feedback signals using TL pulse. Singlet (green squares) and triplet (blue circles) show slightly different behaviour resulting in a decreasing ratio $\mathrm{T} / \mathrm{S}$ with pulse intensity. The grey area indicates the actinic energy regime used by the algorithm in the course of the optimisation.

nals. However, it is evident that the difference between the two signals is shows a jump with the initial fall of the actinic energy and then slowly expands along the learning curve.

To further investigate the importance of the pulse intensity to the control, we conducted intensity dependence measurements using TL pulse with varying intensities. Figure 8.9 shows how the magnitude of the $S$ and $T$ signals depend on the pump intensity with a TL pulse. A difference in the shape of the saturation curves of $\mathrm{S}$ (squares) and $\mathrm{T}$ (circles) is reflected in the fitness value as an increase of the ratio $\mathrm{T} / \mathrm{S}$ (triangles) with decreasing pump intensity. This is in good agreement with what was observed in the T/S optimal control experiments: an initial drop in the pump intensity to some $47 \%$ of the initial energy of $500 \mathrm{~nJ} /$ pulse results in a step-wise increase of the fitness value in the beginning of the optimisation. However, quantitatively the jump from 500 to $235 \mathrm{~nJ} /$ pulse with TL pulse causes a larger $(\sim 17 \%)$ jump in the fitness compared to the one observed in the optimisation and the repeated measurement ( $\sim 6 \%$, see Figure 8.7 and 8.8). Further, as is shown in Figure 8.9 the fitness variation of the TL pulse in the actinic energy range of the optimisation is less than $4 \%$ and cannot therefore be behind the learning process. This indicates that the actual shape of the pulse bears importance implying the involvement of more complicated mechanisms than signal saturation.

\section{Open-loop control}

Open-loop phase scans were performed using the amount linear chirp and the spectral location of a $\pi$ phase steps as variables. First, we tested how a linear chirp influences the triplet and singlet yields. Panel a of Figure 8.10 shows how $\mathrm{S}$ and T decrease similarly when introducing more negative chirp. Positive chirp leads to a different behaviour: $\mathrm{S}$ remains constant whereas $\mathrm{T}$ grows gradually. This variation can be 

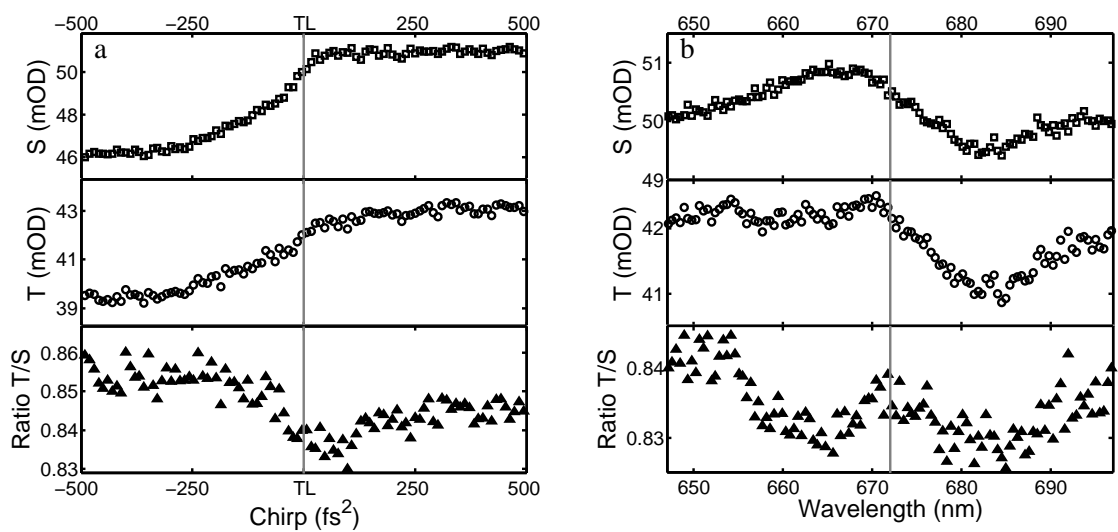

Figure 8.10: Open-loop control results. Panel a: Chirp scan, the feedback signals $\mathrm{S}$ (green squares), $\mathrm{T}$ (blue circles), and the T/S ratio when a linear chirp is scanned from negative to positive. The grey line indicates a TL pulse. Panel b: $\pi$-phase jump scan where the feedback signals $\mathrm{S}$ (green squares), $\mathrm{T}$ (blue circles), and the $\mathrm{T} / \mathrm{S}$ ratio are measured when the spectral position of a step function having a $\pi$ phase jump is scanned over the pump spectrum. The grey line indicates the peak of the resonance.

seen in the asymmetric shape of the ratio (triangles). The overall shape of the chirpscan traces points to a pump-dump mechanism that reduces the population on the excited state demonstrated previously in a study on the fluorescence emission yield of the green fluorescent protein (GFP) [109]. As the initially excited wavepacket slides down the potential energy surface of the first excited state, a negatively chirped pulse can dump it back to the ground state whereas a positively chirped pulse is not able to produce such an effect, or might even reduce it compared to TL pulse. However, this well established mechanism does not explain the different behaviour of the two channels i.e. the gradual growth that was observed for the $\mathrm{T}$ trace. Therefore, we also tested how the chirp scan results react when the pulse intensity is varied (data not shown). Unlike in the GFP study, the same overall features remained (less $\mathrm{S}$ and $\mathrm{T}$ with negative chirp), but the difference between $\mathrm{T}$ and $\mathrm{S}$ vanished with reduced intensity. This could be an indication of a multiphoton process involving higher-lying excited states. The evolution of the wavepacket on the potential energy surface of the first excited state can vary the transition probabilities of possible sequential twophoton absorption processes. By following this evolution a chirped pulse can enhance the yield of such processes, which then lead to more efficient triplet formation.

To further test the involvement of multiphoton processes in the control of the yields of $\mathrm{T}$ and $\mathrm{S}$ we used $\pi$-phase-jump scanning where the spectral position of a stepfunction with an amplitude of $\pi$ is scanned over the pump spectrum while recording the feedback signals. The results in Panel $\mathrm{b}$ of Figure 8.10 show that the $\mathrm{T}$ and $\mathrm{S}$ signals are dependent on the location of the phase jump. The overall shape of the $\mathrm{S}$ and $\mathrm{T}$ traces resembles the imaginary part of resonance that describes absorption. 
The phase profile of the pulse modulates the population transfer by compensating the natural phase flip occurring at resonance. Furthermore, the ratio T/S has a different shape having a maximum at the peak of the absorption band at $672 \mathrm{~nm}$, and U-shaped features symmetrically around it. The ratio trace (black triangles) bears a striking resemblance to previous results on multiphoton transitions [160]. The T/S trace is an intermediate example between a narrow atomic transition and a broad molecular transition of a multiphoton control curve. Qualitatively this makes sense as the width of the $\mathrm{ZnPc}$ absorption band is between the widths of Cesium and Coumarin $6 \mathrm{H}$ used in reference [160] In conclusion, the $\pi$-phase jump scan supports the hypothesis that multiphoton processes are involved in the control mechanism. Finally, it has previously been shown that unlike in the case of nonresonant two-photon absorption TL pulses are not optimal for two-photon transitions involving an intermediate resonant state [161]. Considering this together with the results from the chirp and $\pi$-jump scans we propose a control mechanism that utilises multiphoton pathways to higherlying states from where the inter-system crossing is more probable, thus leading to the enhancement of the triplet yield. Similar mechanism has also been proposed in a previous study on the triplet yield in $\beta$-carotene by Buckup et al. [53].

\subsection{Conclusions and outlook}

We conclude that by combining fluorescence and pump-probe measurements with global analysis of the data we can describe the excited-state dynamics of ZnPc in detail. Once the fluorescence lifetime and quantum yield is known, careful transientabsorption measurements combined with global analyses provide information about the kinetics of the molecular system, ranging from femtosecond to nanosecond time scales. With this, we are able to resolve the lifetimes and quantum yields of fluorescence, internal conversion, and inter-system crossing processes, determine the species-associated spectra belonging to the excited states, and extract dynamics due to solvation. We suggest a new energy model, comprising newly-resolved excited states in the singlet manifold accessed by the probe pulse.

The presented optimal control experiments together with the complementary intensity studies show that it is possible to manipulate the triplet yield of the model photodrug $\mathrm{ZnPc}$, and further that the control mechanisms utilise more elaborate features of the optimal control field than merely the pulse intensity. Based on the openloop scans we propose that the control mechanism involves a sequential resonant twophoton transition to a higher-lying state, which leads to an increased probability of inter-system crossing to the triplet manifold. We note that these ideas need yet to be better substantiated by more analysis as well as new experiments. However, these further studies will greatly benefit from these preliminary results. For instance, the parameterisations for serch space reduction could include higher order chirp terms as well as phase steps. The future studies will also take advantage of the emission properties of the photosensitiser and the actual target molecule singlet oxygen. In a simple emission detection scheme we will optimise the relative yield of the photosensitised singlet oxygen fluorescence and the $\mathrm{ZnPc}$ fluorescence detecting the singlet oxygen fluorescence at $\sim 1270 \mathrm{~nm}$ and photosensitiser fluorescence around $690 \mathrm{~nm}$. 



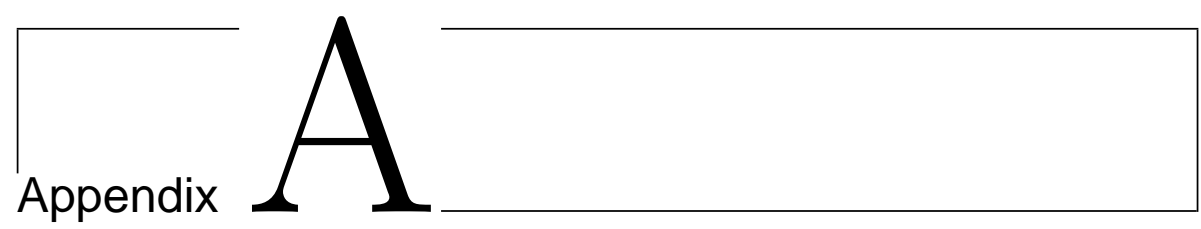

\section{Appendix}

\section{Nonlinear optical processes}

Nonlinear optical processes inside the NOPA ${ }^{\mathrm{TM}}$ box as well as elsewhere in the setup are an essential part of the experiments and are therefore introduced in this Appendix. In order for nonlinear processes to occur, high enough intensities are required, the light entering the media should be coherent, the media must have the right properties, and the phase-matching condition must be satisfied. Hence, in every-day life we don't observe nonlinear optical processes happening. However, the high intensities and coherent nature of ultra-short laser pulses fill these requirements, and therefore cause nonlinear processes readily, especially when coupled into carefully selected and aligned birefringent crystals. There are several textbooks that describe nonlinear optics in depth and for more detailed discussion the reader is referred to the existing literature [162, 163].

Generally, nonlinear optical processes occur when the response of the medium to the electric field of the light becomes nonlinear. In other words, when the induced polarisation $P(t)$ in the medium has contributions from the higher order terms of the Taylor expansion

$$
P(t) \propto \chi^{(1)} P(t)+\chi^{(2)} P^{2}(t)+\chi^{(3)} P^{3}(t)+\ldots .
$$

We now consider the case where we have two fields $E_{1}$ and $E_{2}$ present:

$$
E(t)=E_{1} \exp \left(i \omega_{1} t\right)+E_{1}^{*} \exp \left(-i \omega_{1} t\right)+E_{2} \exp \left(i \omega_{2} t\right)+E_{2}^{*} \exp \left(-i \omega_{2} t\right),
$$

which can lead to so-called $\chi^{(2)}$, or three-wave mixing processes:

$$
\begin{aligned}
& P(t) \propto \quad E_{1}^{2} \exp \left(2 i \omega_{1} t\right)+\text { c.c. }+ \\
& E_{2}^{2} \exp \left(2 i \omega_{2} t\right)+c . c .+ \\
& E_{1} E_{2} \exp \left(2 i\left(\omega_{1}+\omega_{2} t\right)+\text { c.c. }+\right. \\
& E_{1} E_{2} \exp \left(2 i\left(\omega_{1}-\omega_{2} t\right)+\text { c.c. }+\right. \\
& 2 E_{1}^{2}+2 E_{2}^{2},
\end{aligned}
$$


where the corresponding processes are called SHG ( $E_{1}$ field), SHG ( $E_{2}$ field), sumfrequency generation (SFG), difference-frequency generation (DFG) and optical dc rectification $(\mathrm{OR})$.

Nonlinear processes are typically realised in the laboratory by coupling a beam into a birefringent crystal, for instance $\mathrm{BBO}$, that has an extraordinary and ordinary axes that have different refractive indices for different polarisations. This makes it possible to find a certain phase-matching angle where the induced polarisation and the second-harmonic frequencies have the same effective refractive indices and the process may continue throughout the crystal length. In other words, at the phasematching angle the phase-matching condition is satisfied. For instance,

$$
n_{p} k_{p}=n_{s} k_{s}+n_{s} k_{s}
$$

is the phase-matching condition for DFG. The fields, refractive indices, and wave vectors $k$ have been renamed as pump, signal, and idler, corresponding to the high frequency field, intermediate frequency field and the low-frequency field.

An important phenomenon called the group-velocity mismatch (GVM) due to the general dispersive property of matter must be considered. GVM occurs because fields having higher frequencies see generally a higher refractive index and therefore travel slower in the crystal than fields having lower frequencies. This phenomenon results in a so-called bandwidth limit, which depends on the crystal thickness, the material used, and the wavelength of the initial field. In practice, we are faced with a compromise: shorter the pulses thinner the crystal. But, thinner the crystal, lower will the output intensity be.

\section{Optical parametric amplification, OPA}

OPA is a special example of a $\chi^{(2)}$ process, where the intensity of the signal field is weak. A field with the difference frequency between the pump and seed fields $\omega_{i}$ begins to form in the front end of the crystal. This field interacts with the pump field having a frequency $\omega_{p}$ and generates the difference frequency $\omega_{s}$. As the beam propagates, these interactions are repeated and the $\omega_{s}$ and $\omega_{i}$ fields get amplified gaining energy from the $\omega_{p}$ field.

In other words, in OPA, the high-energy pump photons $\omega_{p}$ from the SHG are split into one signal photon $\omega_{s}$ (visible) and one idler $\omega_{i}$ (near-infrared, NIR) photon each. The conservation of energy gives us:

$$
\omega_{p}=\omega_{s}+\omega_{i}
$$

where $\omega_{p}, \omega_{s}$, and $\omega_{i}$ are the pump, signal (and seed), and idler frequencies, respectively.

As mentioned above, the phase velocities of pump, signal and idler are matched by proper orientation of the birefringent crystal for efficient conversion. Since the peak intensities of the ultra-fast pulses are very high, even when the pulse energies are relatively low, it is possible to reach considerable gain in the OPA process without destroying the crystal. OPA is schematically illustrated in Panel a of Figure A.1. Unfortunately, the three fields propagate with different speeds inside the crystal, and GVM introduces a limit for how broad spectra and how short pulses can be amplified. 
a

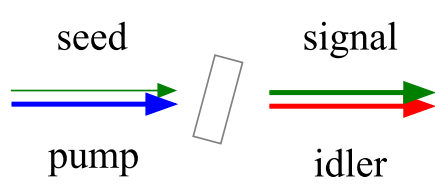

$\mathrm{b}$

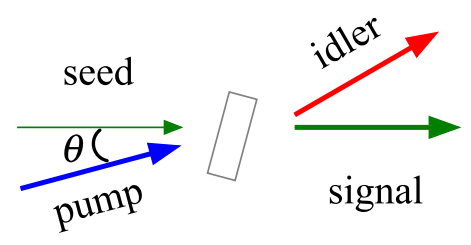

Figure A.1: Schematic representation of the OPA and NOPA processes. Panel a: The pump photons are split into signal and idler photons, while the splitting is guided by the seed photons. Panel b: The noncollinear arrangement NOPA with the noncollinearity angle $\theta$.

As a result of GVM the typical practical limit for the pulse length of the amplified signal field is in the order of $\sim 100 \mathrm{fs}$ for the collinear configuration in a 1-mm BBO crystal.

The aforementioned limit can be overcome by introducing a noncollinearity angle between the seed and the pump beams [164]. This means that an angle can be found between the pump and the seed, so that the projection of the idler group velocity matches the signal group velocity.

$$
\mathrm{GV}\left(\omega_{2}\right) \cos \theta=\mathrm{GV}\left(\omega_{1}\right),
$$

where $\theta$ is the angle between the two input beams (see Figure A.1). This makes it possible to amplify very broad spectra and as a consequence, pulses out of NOPA can be compressed to pulse lengths as short as $10 \mathrm{fs}[59]$.

\section{White-light continuum, WLC}

WLC is used inside the NOPA ${ }^{\mathrm{TM}}$ box to provide the seed beam for the first OPA step as well as in the setup to provide the broad-band probe pulses. Higher order terms of the induced polarisation (Equation A.1) are required to describe the WLC generation. A major contribution occurs due to a third-order nonlinear optical process, which is usually called optical Kerr effect or, when the effect arises from a single pulse itself, self-phase modulation. Generally, in any media, if intensity of the incident field is high enough, the refractive index becomes a function of the incident intensity.

$$
n(I)=n_{1}+n_{2} I+\ldots,
$$

In WLC generation, self-focusing readily causes the beam to break up into filaments that interfere and make the white light output extremely unstable. In practice, this limits the amount of energy that can be used to feed the process. Therefore, In order to create as much and as stable as possible WLC the energy, beam waist (depth of the focus) and the position of the focus in the crystal must be optimised. This is done with a gradient neutral-density filter, an adjustable aperture, and moving the focusing element or the crystal carefully with a so-called $\mu \mathrm{m}$ stage. 



\section{Bibliography}

[1] A. Sinha, M. Hsiao, F. Crim, Bond-selected bimolecular chemistry:H+HOD(4$\nu$-OH)->OD+H2, J Chem Phys 92 (1990) 6333-6335.

[2] R. Vander Wal, J. Scott, F. Crim, Selectively breaking the O-H bond in HOD, J Chem Phys 92 (1990) 803-805.

[3] P. Brumer, M. Shapiro, Control of unimolecular reactions using coherent light, Chem Phys Lett 126 (1986) 541-546.

[4] U. Gaubatz, P. Rudecki, M. Becker, S. Schiemann, M. Kulz, K. Bergmann, Population switching between vibrational levels in molecular-beams, Chem Phys Lett 149 (1988) 463-468.

[5] D. Tannor, S. Rice, Control of selectivity of chemical reaction via control of wave packet evolution, J Chem Phys 83 (1985) 5013-5018.

[6] D. Tannor, R. Kosloff, S. Rice, Coherent pulse sequence induced control of selectivity of reactions - exact quantum-mechanical calculations, J Chem Phys 85 (1986) 5805-5820.

[7] A. Peirce, M. Dahleh, H. Rabitz, Optimal-control of quantum-mechanical systems - existence, numerical approximation and applications, Phys Rev A: At , Mol Opt Phys 37 (1988) 4950-4964.

[8] R. S. Judson, H. Rabitz, Teaching lasers to control molecules., Phys Rev Lett 68 (1992) 1500-1503.

[9] A. M. Weiner, D. E. Leaird, G. P. Wiederrecht, K. A. Nelson, Femtosecond pulse sequences used for optical manipulation of molecular-motion, Science 247 (1990) 1317-1319.

[10] T. Hornung, R. Meier, M. Motzkus, Optimal control of molecular states in a learning loop with a parameterization in frequency and time domain, Chem Phys Lett 326 (2000) 445-453. 
[11] T. Hornung, R. Meier, R. de Vivie-Riedle, M. Motzkus, Coherent control of the molecular four-wave-mixing response by phase and amplitude shaped pulses, Chem Phys 267 (2001) 261-276.

[12] A. Assion, T. Baumert, M. Bergt, T. Brixner, B. Kiefer, V. Seyfried, M. Strehle, G. Gerber, Control of chemical reactions by feedback-optimized phase-shaped femtosecond laser pulses, Science 282 (1998) 919-922.

[13] G. Vogt, P. Nuernberger, T. Brixner, G. Gerber, Femtosecond pump-shapeddump quantum control of retinal isomerization in bacteriorhodopsin, Chem Phys Lett 433 (2006) 211-215.

[14] V. I. Prokhorenko, A. M. Nagy, S. A. Waschuk, L. S. Brown, R. R. Birge, R. J. D. Miller, Coherent control of retinal isomerization in bacteriorhodopsin, Science 313 (2006) 1257-1261.

[15] N. H. Bonadeo, J. Erland, D. Gammon, D. Park, D. S. Katzer, D. G. Steel, Coherent optical control of the quantum state of a single quantum dot, Science 282 (1998) 1473-1476.

[16] R. Bartels, S. Backus, E. Zeek, L. Misoguti, G. Vdovin, I. P. Christov, M. M. Murnane, H. C. Kapteyn, Shaped-pulse optimization of coherent emission of high-harmonic soft x-rays, Nature 406 (2000) 164-166.

[17] D. Meshulach, Y. Silberberg, Coherent quantum control of two-photon transitions by a femtosecond laser pulse, Nature 396 (1998) 239-242.

[18] P. V. Kamat, Meeting the clean energy demand: Nanostructure architectures for solar energy conversion, J Phys Chem C 111 (2007) 2834-2860.

[19] D. Gust, T. A. Moore, A. L. Moore, Mimicking photosynthetic solar energy transduction, Acc Chem Res 34 (2001) 40-48.

[20] T. J. Dougherty, C. J. Gomer, B. W. Henderson, G. Jori, D. Kessel, M. Korbelik, J. Moan, Q. Peng, Photodynamic therapy, J Natl Cancer Inst 90 (1998) 889905.

[21] H. Rabitz, R. de Vivie-Riedle, M. Motzkus, K. Kompa, Chemistry - whither the future of controlling quantum phenomena?, Science 288 (2000) 824-828.

[22] T. Brixner, N. H. Damrauer, P. Niklaus, G. Gerber, Photoselective adaptive femtosecond quantum control in the liquid phase, Nature 414 (2001) 57-60.

[23] N. J. Turro, Modern Molecular Photochemistry, University Science Books, U.S., 1991.

[24] J. M. Hollas, Modern Spectroscopy, John Wiley \& Sons, 2003.

[25] P. W. Atkins, R. S. Friedman, Molecular Quantum Mechanics, Oxford University Press, 2004.

[26] P. W. Atkins, Physical chemistry, Oxford University Press, 1993. 
[27] L. J. Fogel, A. J. Owens, M. J. Walsh, Artificial Intelligence through Simulated Evolution, John Wiley \& Sons, New York, 1966.

[28] J. H. Holland, Adaptation in Natural and Artificial Systems: An Introductory Analysis with Applications to Biology, Control and Artificial Intelligence, MIT Press, Cambridge, 1992.

[29] I. Rechenberg, Evolutionsstrategie: Optimierung technischer Systeme nach Prinzipien der biologischen Evolution, Frommann-Holzboog Verlag, Stuttgart, 1973.

[30] H. P. Schwefel, Numerical Optimization of Computer Models, John Wiley \& Sons, Inc., New York, 1981.

[31] T. Bäck, H. P. Schwefel, An overview of evolutionary algorithms for parameter optimization, Evolutionary Computation 1 (1993) 1-23.

[32] D. B. Fogel, An introduction to simulated evolutionary optimization, IEEE transaction on neural networks 5 (1994) 3-14.

[33] H. P. Schwefel, Evolution and Optimum Seeking, Wiley, New York, 1995.

[34] A. Ostermeier, A. Gawelczyk, N. Hansen, Step-size adaptation based on nonlocal use of selection information, in: H.-M. V. et al. (Ed.), Parallel Problem Solving from Nature - PPSN IV, Springer, Berlin, 1994, pp. 189-198.

[35] N. Hansen, A. Ostermeier, Completely derandomized self-adaptation in evolution strategies, Evolutionary Computation 9 (2001) 159-195.

[36] R. Trebino, K. W. DeLong, D. N. Fittinghoff, J. N. Sweetser, M. A. Krumbügel, B. A. Richman, Measuring ultrashort laser pulses in the time-frequency domain using frequency-resolved optical gating, Rev Sci Instrum 68 (1997) 3277-3295.

[37] V. V. Lozovoy, I. Pastirk, M. Dantus, Multiphoton intrapulse interference. iv. ultrashort laser pulse spectral phase characterization and compensation, Opt Lett 29 (2004) 775-777.

[38] J. M. Geremia, W. S. Zhu, H. Rabitz, Incorporating physical implementation concerns into closed loop quantum control experiments, J Chem Phys 113 (2000) 10841-10848.

[39] S. M. Weber, A. Lindinger, M. Plewicki, C. Lupulescu, F. Vetter, L. Wöste, Temporal and spectral optimization course analysis of coherent control experiments, Chem Phys 306 (2004) 287-293.

[40] J. L. White, B. J. Pearson, P. H. Bucksbaum, Extracting quantum dynamics from genetic learning algorithms through principal control analysis, J Phys B At Mol Opt Phys 37 (2004) L399-L405.

[41] M. A. Montgomery, R. R. Meglen, N. H. Damrauer, General method for the dimension reduction of adaptive control experiments, J Phys Chem A 110 (2006) 6391-6394. 
[42] D. Zeidler, S. Frey, K. L. Kompa, M. Motzkus, Evolutionary algorithms and their application to optimal control studies, Phys Rev A: At , Mol Opt Phys 64 (2001) 023420-023432.

[43] J. L. Herek, W. Wohlleben, R. J. Cogdell, D. Zeidler, M. Motzkus, Quantum control of energy flow in light harvesting, Nature 417 (2002) 533-535.

[44] W. Wohlleben, T. Buckup, J. L. Herek, M. Motzkus, Coherent control for spectroscopy and manipulation of biological dynamics, ChemPhysChem 6 (2005) 850-857.

[45] D. Cardoza, F. Langhojer, C. Trallero-Herrero, O. L. A. Monti, T. Weinacht, Changing pulse-shape basis for molecular learning control, Phys Rev A: At, Mol Opt Phys 70 (2004) -.

[46] F. Langhojer, D. Cardoza, M. Baertschy, T. Weinacht, Gaining mechanistic insight from closed loop learning control: The importance of basis in searching the phase space, J Chem Phys 122 (2005) -.

[47] A. F. Bartelt, T. Feurer, L. Wöste, Understanding optimal control results by reducing the complexity, Chem Phys 318 (2005) 207-216.

[48] A. Lindinger, S. M. Weber, C. Lupulescu, F. Vetter, M. Plewicki, A. Merli, L. Wöste, A. F. Bartelt, H. Rabitz, Revealing spectral field features and mechanistic insights by control pulse cleaning, Phys Rev A: At, Mol Opt Phys 71.

[49] A. Lindinger, S. M. Weber, A. Merli, F. Sauer, M. Plewicki, L. Wöste, Optimal control methods applied on the ionization processes of alkali dimers, J Photochem Photobiol Chem 180 (2006) 256-261.

[50] A. Bartelt, A. Lindinger, C. Lupulescu, S. Vajda, L. Wöste, One parameter fs-pulse form control on NaK and Na2K, Phys Chem Chem Phys 5 (2003) 3610-3615.

[51] J. Hauer, H. Skenderovic, K. L. Kompa, M. Motzkus, Enhancement of Raman modes by coherent control in beta-carotene, Chem Phys Lett 421 (2006) 523528.

[52] J. Hauer, T. Buckup, M. Motzkus, Pump-degenerate four wave mixing as a technique for analyzing structural and electronic evolution: Multidimensional time-resolved dynamics near a conical intersection, J Phys Chem A 111 (2007) 10517-10529.

[53] T. Buckup, T. Lebold, A. Weigel, W. Wohleben, M. Motzkus, Singlet vs triplet dynamics of beta-carotene studied by quantum control spectroscopy, J Photochem Photobiol Chem 180 (2006) 314-321.

[54] V. V. Lozovoy, M. Dantus, Coherent control in femtochemistry, ChemPhysChem 6 (2005) 1970-2000. 
[55] J. M. Dela Cruz, I. Pastirk, M. Comstock, V. V. Lozovoy, M. Dantus, Use of coherent control methods through scattering biological tissue to achieve functional imaging, Proc Natl Acad Sci USA 101 (49) (2004) 16996-17001.

[56] N. Dudovich, D. Oron, Y. Silberberg, Single-pulse coherently controlled nonlinear Raman spectroscopy and microscopy, Nature 418 (2002) 512-514.

[57] B. von Vacano, M. Motzkus, Time-resolving molecular vibration for microanalytics: single laser beam nonlinear Raman spectroscopy in simulation and experiment, Phys Chem Chem Phys 10 (2008) 681-691.

[58] T. Wilhelm, J. Piel, E. Riedle, Sub-20-fs pulses tunable across the visible from a blue-pumped single-pass noncollinear parametric converter, Opt Lett 22 (1997) 1494-1496.

[59] E. Riedle, M. Beutter, S. Lochbrunner, J. Piel, S. Schenkl, S. Sporlein, W. Zinth, Generation of 10 to $50 \mathrm{fs}$ pulses tunable through all of the visible and the nir, Appl Phys B: Lasers Opt 71 (2000) 457-465.

[60] J. Piel, M. Beutter, E. Riedle, 20-50-fs pulses tunable across the near infrared from a blue-pumped noncollinear parametric amplifier, Opt Lett 25 (2000) 180 182.

[61] A. M. Weiner, Femtosecond pulse shaping using spatial light modulators, Rev Sci Instrum 71 (2000) 1929-1960.

[62] O. Shir, C. Siedschlag, T. Bäck, M. Vrakking, Evolutionary algorithms in the optimization of dynamic molecular alignment, in: G. G. Y. et al. (Ed.), IEEE Congress on Evolutionary Computation, IEEE Press, Vancouver, 2006.

[63] O. M. Shir, T. Bäck, The second harmonic generation case-study as a gateway for ES to quantum control problems, in: H. Lipson (Ed.), Genetic and Evolutionary Computation Conference, ACM, 2007.

[64] R. Trebino, Frequency-Resolved Optical Gating: The Measurement of Ultrashort Laser Pulses, Kluwer Academic, Dordrecht, 2002.

[65] N. Hansen, A. Ostermeier, A. Gawelczyk, On the adaptation of arbitrary normal mutation distributions in evolution strategies: The generating set adaptation, in: L. Eshelman (Ed.), Sixth International Conference on Genetic Algorithms, Morgan Kaufmann, San Francisco, 1995.

[66] H.-G. Beyer, Evolutionary Algorithms in Noisy Environments: Theoretical Issues and Guidelines for Practice, Comput Meth Appl Mech Eng 186 (2000) 239-267.

[67] G. Galilei, D. Stillman, Two New Sciences: Including Centers of Gravity Force of Percussion, University Of Wisconsin Press, 1974.

[68] J. M. Keynes, The Collected Writings of J. M. Keynes, Vol. VII, Macmillan for the Royal Economic Society, London, 1973, Ch. The General Theory of Employment, Interest and Money. 
[69] G. W. Greenwood, Intrinsic evolution of safe control strategies for autonomous spacecraft, IEEE Trans Aero Electron Syst 40 (1) (2004) 236-246.

[70] Z. Fan, J. Wang, S. Achiche, E. Goodman, R. Rosenberg, Structured synthesis of mems using evolutionary approaches, Applied Soft Computing 8 (1) (2008) 579-589.

[71] S. Wannarumon, E. L. J. Bohez, K. Annanon, Aesthetic evolutionary algorithm for fractal-based user-centered jewelry design, AI EDAM 22 (1) (2008) 19-39.

[72] Z. G. Diamantis, D. T. Tsahalis, I. Borchers, Optimization of an active noise control system inside an aircraft, based on the simultaneous optimal positioning of microphones and speakers, with the use of a genetic algorithm, Comput Optim Appl 23 (1) (2002) 65-76.

[73] J. M. Carmena, N. Kampchen, D. Kim, J. C. T. Hallam, Artificial ears for a biomimetic sonarhead: From multiple reflectors to surfaces, Artif Life 7 (2) (2001) 147-169.

[74] S. Rice, M. Zhao, Optical control of molecular dynamics, John Wiley, New York, 2000.

[75] T. Brixner, G. Gerber, Quantum control of gas-phase and liquid-phase femtochemistry, ChemPhysChem 4 (2003) 418-438.

[76] M. Dantus, V. V. Lozovoy, Experimental coherent laser control of physicochemical processes, Chem Rev 104 (2004) 1813-1859.

[77] A. Macpherson, P. Liddell, D. Kuciauskas, D. Tatman, T. Gillbro, D. Gust, T. Moore, A. Moore, Ultrafast energy transfer from a carotenoid to a chlorin in a simple artificial photosynthetic antenna, J Phys Chem B 106 (2002) 94249433.

[78] J. Savolainen, N. Dijkhuizen, R. Fanciulli, P. A. Liddell, D. Gust, T. A. Moore, A. L. Moore, J. Hauer, T. Buckup, M. Motzkus, J. L. Herek, Ultrafast energy transfer dynamics of a bioinspired dyad molecule, J Phys Chem B 112 (2008) 2678-2685.

[79] G. McDermott, S. M. Prince, A. A. Freer, A. M. Hawthornthwaite-Lawless, M. Z. Papiz, R. J. Cogdell, N. W. Isaacs, Crystal structure of an integral membrane light-harvesting complex from photosynthetic bacteria, Nature 374 (1995) 517-521.

[80] T. Polivka, V. Sundstrom, Ultrafast dynamics of carotenoid excited states-from solution to natural and artificial systems, Chem Rev 104 (2004) 2021-2072.

[81] V. Sundstrom, T. Pullerits, R. van Grondelle, Photosynthetic light-harvesting: Reconciling dynamics and structure of purple bacterial $\mathrm{LH} 2$ reveals function of photosynthetic unit, J Phys Chem B 103 (1999) 2327-2346. 
[82] A. N. Macpherson, J. B. Arellano, N. J. Fraser, R. J. Cogdell, T. Gillbro, Efficient energy transfer from the carotenoid S2 state in a photosynthetic lightharvesting complex, Biophys J 80 (2001) 923-930.

[83] T. Polivka, D. Zigmantas, J. L. Herek, Z. He, T. Pascher, T. Pullerits, R. J. Cogdell, H. A. Frank, V. Sundstrom, The carotenoid S-1 state in LH2 complexes from purple bacteria rhodobacter sphaeroides and rhodopseudomonas acidophila: S-1 energies, dynamics, and carotenoid radical formation, J Phys Chem B 106 (2002) 11016-11025.

[84] D. Gust, T. A. Moore, A. L. Moore, P. A. Liddell, Synthesis of carotenoporphyrin models for photosynthetic energy and electron transfer, in: L. Packer (Ed.), Carotenoids Part A: Chemistry, Separation, Quantitation, and Antioxidation, Vol. 213 of Methods in Enzymology, Academic Press, 1992, pp. 87-100.

[85] D. Polli, G. Cerullo, G. Lanzani, S. De Silvestri, H. Hashimoto, R. J. Cogdell, Carotenoid-bacteriochlorophyll energy transfer in LH2 complexes studied with 10-fs time resolution, Biophys J 90 (2006) 2486-2497.

[86] W. Fuss, Y. Haas, S. Zilberg, Twin states and conical intersections in linear polyenes, Chem Phys 259 (2000) 273-295.

[87] R. Berera, C. Herrero, L. H. M. van Stokkum, M. Vengris, G. Kodis, R. E. Palacios, H. van Amerongen, R. van Grondelle, D. Gust, T. A. Moore, A. L. Moore, J. T. M. Kennis, A simple artificial light-harvesting dyad as a model for excess energy dissipation in oxygenic photosynthesis, Proc Natl Acad Sci USA 103 (2006) 5343-5348.

[88] E. Papagiannakis, J. T. M. Kennis, I. H. M. van Stokkum, R. J. Cogdell, R. van Grondelle, An alternative carotenoid-to-bacteriochlorophyll energy transfer pathway in photosynthetic light harvesting, Proc Natl Acad Sci USA 99 (2002) 6017-6022.

[89] D. S. Larsen, E. Papagiannakis, I. H. M. van Stokkum, M. Vengris, J. T. M. Kennis, R. van Grondelle, Excited state dynamics of beta-carotene explored with dispersed multi-pulse transient absorption, Chem Phys Lett 381 (2003) 733-742.

[90] C. C. Gradinaru, J. T. M. Kennis, E. Papagiannakis, I. H. M. van Stokkum, R. J. Cogdell, G. R. Fleming, R. A. Niederman, R. van Grondelle, An unusual pathway of excitation energy deactivation in carotenoids: Singlet-to-triplet conversion on an ultrafast timescale in a photosynthetic antenna, Proc Natl Acad Sci USA 98 (2001) 2364-2369.

[91] A. R. Holzwarth, Data analysis of time-resolved measurements, in: J. Amesz, A. J. Hoff (Eds.), Biophysical Techniques in Photosynthesis, Vol. 3 of Advances in Biophysics, Kluwert Academic Publishers, Dordrecht, 1996, pp. 75-92.

[92] I. H. M. van Stokkum, D. S. Larsen, R. van Grondelle, Global and target analysis of time-resolved spectra, Biochimica et Biophysica Acta (BBA) - Bioenergetics 1657 (2004) 82-104. 
[93] W. Wohlleben, T. Buckup, J. L. Herek, R. J. Cogdell, M. Motzkus, Multichannel carotenoid deactivation in photosynthetic light harvesting as identified by an evolutionary target analysis, Biophys J 85 (2003) 442-450.

[94] R. Fanciulli, L. Willmes, J. Savolainen, P. van der Walle, T. Bäck, J. L. Herek, Evolution strategies for laser pulse compression, in: N. Monmarchè, E.-G. Talbi, P. Collet, M. Schoenauer, E. Lutton (Eds.), 8th International Conference on Artificial Evolution, Vol. 4926 of Lecture Notes in Computer Science, Springer, Tours, 2007.

[95] P. O. Andersson, T. Gillbro, Photophysics and dynamics of the lowest excited singlet state in long substituted polyenes with implications to the very longchain limit, J Chem Phys 103 (1995) 2509-2519.

[96] T. Buckup, J. Savolainen, W. Wohlleben, J. L. Herek, H. Hashimoto, R. R. B. Correia, M. Motzkus, Pump-probe and pump-deplete-probe spectroscopies on carotenoids with $n=9-15$ conjugated bonds, J Chem Phys 125 (2006) 1945057.

[97] E. Papagiannakis, I. H. M. van Stokkum, M. Vengris, R. J. Cogdell, R. van Grondelle, D. S. Larsen, Excited-state dynamics of carotenoids in lightharvesting complexes. 1. exploring the relationship between the $S_{1}$ and $S^{*}$ states, J Phys Chem B 110 (2006) 5727-5736.

[98] Y.-X. Yan, E. B. Gamble, Jr., K. A. Nelson, Impulsive stimulated scattering: General importance in femtosecond laser pulse interactions with matter, and spectroscopic applications, J Chem Phys 83 (1985) 5391-5399.

[99] W. Wohlleben, T. Buckup, H. Hashimoto, R. Cogdell, J. Herek, M. Motzkus, Pump-deplete-probe spectroscopy and the puzzle of carotenoid dark states, J Phys Chem B 108 (2004) 3320-3325.

[100] M. Wollenhaupt, V. Engel, T. Baumert, Femtosecond laser photoelectron spectroscopy on atoms and small molecules: Prototype studies in quantum control, Annu Rev Phys Chem 56 (2005) 25-56.

[101] T. Buckup, W. Wohlleben, J. Savolainen, B. Heinz, H. Hashimoto, R. J. Cogdell, J. L. Herek, M. Motzkus, Energy flow in carotenoids, studied with pump-deplete-probe, multiphoton- and coherent control spectroscopy, in: Ultrafast Phenomena XIV, Springer, Berlin, 2005, pp. 368-370.

[102] G. Cerullo, G. Lanzani, M. Zavelani-Rossi, S. De Silvestri, Early events of energy relaxation in all-trans-beta-carotene following sub-10 fs optical-pulse excitation, Phys Rev B 63 (2001) 241104-4.

[103] R. Shimada, K. Hideaki, H.-o. Hamaguchi, Molecular near-field effect and intensity enhancement of solvent modes in resonance hyper-Raman scattering, $\mathbf{J}$ Raman Spectrosc 37 (2006) 469-471.

[104] L. Dhar, J. Rogers, K. A. Nelson, Time-resolved vibrational spectroscopy in the impulsive limit, Chem Rev 94 (1994) 157-193. 
[105] D. Gust, T. A. Moore, A. L. Moore, Molecular mimicry of photosynthetic energy and electron-transfer, Acc Chem Res 26 (1993) 198-205.

[106] G. Kodis, P. A. Liddell, L. de la Garza, P. C. Clausen, J. S. Lindsey, A. L. Moore, T. A. Moore, D. Gust, Efficient energy transfer and electron transfer in an artificial photosynthetic antenna-reaction center complex, J Phys Chem A 106 (2002) 2036-2048.

[107] D. Kuciauskas, P. A. Liddell, S. Lin, T. E. Johnson, S. J. Weghorn, J. S. Lindsey, A. L. Moore, T. A. Moore, D. Gust, An artificial photosynthetic antennareaction center complex, J Am Chem Soc 121 (1999) 8604-8614.

[108] P. Nuernberger, G. Vogt, T. Brixner, G. Gerber, Femtosecond quantum control of molecular dynamics in the condensed phase, Phys Chem Chem Phys 9 (2007) 2470-2497.

[109] C. Bardeen, V. V. Yakovlev, J. Squier, K. R. Wilson, Quantum control of population transfer in green fluorescent protein by using chirped femtosecond pulses, J Am Chem Soc 120 (1998) 13023-13027.

[110] V. I. Prokhorenko, A. M. Nagy, R. J. D. Miller, Coherent control of the population transfer in complex solvated molecules at weak excitation. an experimental study, J Chem Phys 122 (2005) 184502-11.

[111] T. C. Weinacht, J. L. White, P. H. Bucksbaum, Toward strong field modeselective chemistry, J Phys Chem A 103 (1999) 10166-10168.

[112] B. Dietzek, B. Bruggemann, T. Pascher, A. Yartsev, Mechanisms of molecular response in the optimal control of photoisomerization, Phys Rev Lett 97 (2006) 258301-4.

[113] G. Vogt, G. Krampert, P. Niklaus, P. Nuernberger, G. Gerber, Optimal control of photoisomerization, Phys Rev Lett 94 (2005) 068305-4.

[114] E. Papagiannakis, M. Vengris, L. Valkunas, R. J. Cogdell, R. van Grondelle, D. S. Larsen, Excited-state dynamics of carotenoids in light-harvesting complexes. 2. dissecting pulse structures from optimal control experiments, J Phys Chem B 110 (2006) 5737-5746.

[115] T. Hornung, H. Skenderovic, M. Motzkus, Observation of all-trans-betacarotene wavepacket motion on the electronic ground and excited dark state using degenerate four-wave mixing (DFWM) and pump-DFWM, Chem Phys Lett 402 (2005) 283-288.

[116] J. L. P. Lustres, D. Alexander, L., A. Holzwarth, M. Veiga, Internal conversion in beta-carotene: Strong vibronic coupling from amplitude oscillations of transient absorption bands, Angew Chem Ed 46 (2007) 3758-3761.

[117] W. Fuss, S. Lochbrunner, A. M. Muller, T. Schikarski, W. E. Schmid, S. A. Trushin, Pathway approach to ultrafast photochemistry: potential surfaces, conical intersections and isomerizations of small polyenes, Chem Phys 232 (1998) 161-174. 
[118] N. Dudovich, D. Oron, Y. Silberberg, Single-pulse coherent anti-stokes Raman spectroscopy in the fingerprint spectral region, J Chem Phys 118 (2003) 92089215.

[119] N. F. Scherer, R. J. Carlson, A. Matro, M. Du, A. J. Ruggiero, V. Romerorochin, J. A. Cina, G. R. Fleming, S. A. Rice, Fluorescence-detected wave packet interferometry - time resolved molecular-spectroscopy with sequences of femtosecond phase-locked pulses, J Chem Phys 95 (1991) 1487-1511.

[120] T. Buckup, J. Hauer, C. Serrat, M. Motzkus, Control of excited state population and vibrational coherence with shaped-resonant and near-resonant excitation, J Phys B: At, Mol Opt Phys 41 (2008) 074024-12.

[121] J. Hauer, T. Buckup, M. Motzkus, Enhancement of molecular modes by electronically resonant multipulse excitation: Further progress towards mode selective chemistry, J Chem Phys 125 (2006) 061101-4.

[122] R. L. Christensen, M. G. I. Galinato, E. F. Chu, R. Fujii, H. Hashimoto, H. A. Frank, Symmetry control of radiative decay in linear polyenes: Low barriers for isomerization in the S-1 state of hexadecaheptaene, J Am Chem Soc 129 (2007) 1769-1775.

[123] D. Niedzwiedzki, J. F. Koscielecki, H. Cong, J. O. Sullivan, G. N. Gibson, R. R. Birge, H. A. Frank, Ultrafast dynamics and excited state spectra of open-chain carotenoids at room and low temperatures, J Phys Chem B 111 (21) (2007) 5984-5998.

[124] M. C. DeRosa, R. J. Crutchley, Photosensitized singlet oxygen and its applications, Coord Chem Rev 233 (2002) 351-371.

[125] M. Ochsner, Photophysical and photobiological processes in the photodynamic therapy of tumours, J Photochem Photobiol B Biol 39 (1997) 1-18.

[126] I. J. MacDonald, T. J. Dougherty, Basic principles of photodynamic therapy, J Porphyrins Phthalocyanines 5 (2001) 105-129.

[127] C. N. Lunardi, A. C. Tedesco, Synergic photosensitizers: A new trend in photodynamic therapy, Curr Org Chem 9 (2005) 813-821.

[128] J. W. Owens, R. Smith, R. Robinson, M. Robins, Photophysical properties of porphyrins, phthalocyanines, and benzochlorins, Inorg Chim Acta 279 (1998) 226-231.

[129] C. M. Allen, W. M. Sharman, J. E. Van Lier, Current status of phthalocyanines in the photodynamic therapy of cancer, J Porphyrins Phthalocyanines 5 (2001) 161-169.

[130] S. M. T. Nunes, F. S. Sguilla, A. C. Tedesco, Photophysical studies of zinc phthalocyanine and chloroaluminum phthalocyanine incorporated into liposomes in the presence of additives, Braz J Med Biol Res 37 (2004) 273-284. 
[131] A. Grofcsik, N. Baranyai, I. Bitter, V. Csokai, M. Kubinyi, K. Szegletes, J. Tatai, T. Vidoczy, Triple state properties of tetrasubstituted zinc phthalocyanine derivatives, J Mol Struct 704 (2004) 11-15.

[132] L. Howe, J. Z. Zhang, Ultrafast studies of excited-state dynamics of phthalocyanine and zinc phthalocyanine tetrasulfonate in solution, J Phys Chem A 101 (1997) 3207-3213.

[133] L. Howe, J. Z. Zhang, The effect of biological substrates on the ultrafast excited-state dynamics of zinc phthalocyanine tetrasulfonate in solution, Photochem Photobiol 67 (1998) 90-96.

[134] D. Frackowiak, A. Planner, A. Waszkowiak, A. Boguta, R. M. Ion, K. Wiktorowicz, Yield of intersystem (singlet-triplet) crossing in phthalocyanines evaluated on the basis of a time in resolved photothermal method, J Photochem Photobiol Chem 141 (2001) 101-108.

[135] S. V. Rao, D. N. Rao, Excited state dynamics in phthalocyanines studied using degenerate four wave mixing with incoherent light, J Porphyrins Phthalocyanines 6 (2002) 233-237.

[136] M. Fournier, C. Pepin, D. Houde, R. Ouellet, J. E. van Lier, Ultrafast studies of the excited-state dynamics of copper and nickel phthalocyanine tetrasulfonates: potential sensitizers for the two-photon photodynamic therapy of tumors, Photochem Photobiol Sci 3 (2004) 120-126.

[137] P. S. Vincett, E. M. Voigt, K. E. Rieckhoff, Phosphorescence and fluorescence of phthalocyanines, J Chem Phys 55 (1971) 4131-4140.

[138] Q. H. Zhong, Z. H. Wang, Y. Q. Liu, Q. H. Zhu, F. A. Kong, The ultrafast intramolecular dynamics of phthalocyanine and porphyrin derivatives, J Chem Phys 105 (1996) 5377-5379.

[139] A. Ogunsipe, J. Y. Chen, T. Nyokong, Photophysical and photochemical studies of zinc(II) phthalocyanine derivatives - effects of substituents and solvents, New J Chem 28 (2004) 822-827.

[140] A. Ogunsipe, D. Maree, T. Nyokong, Solvent effects on the photochemical and fluorescence properties of zinc phthalocyanine derivatives, J Mol Struct 650 (2003) 131-140.

[141] A. Ogunsipe, T. Nyokong, Effects of substituents and solvents on the photochemical properties of zinc phthalocyanine complexes and their protonated derivatives, J Mol Struct 689 (2004) 89-97.

[142] M. Gouterman, in: D. Dolphin (Ed.), The Porphyrins, Vol. 3, Academic Press, New York, 1978.

[143] G. Valduga, E. Reddi, G. Jori, R. Cubeddu, P. Taroni, G. Valentini, Steadystate and time-resolved spectroscopic studies on zinc(II)phthalocyanine in liposomes, J Photochem Photobiol B Biol 16 (1992) 331-340. 
[144] K. Lang, J. Mosinger, D. M. Wagnerova, Photophysical properties of porphyrinoid sensitizers non-covalently bound to host molecules; models for photodynamic therapy, Coord Chem Rev 248 (2004) 321-350.

[145] S. M. Bishop, A. Beeby, A. W. Parker, M. S. C. Foley, D. Phillips, The preparation and photophysical measurements of perdeutero zinc phthalocyanine, J Photochem Photobiol Chem 90 (1995) 39-44.

[146] R. Jimenez, G. R. Fleming, P. V. Kumar, M. Maroncelli, Femtosecond solvation dynamics of water, Nature 369 (1994) 471-473.

[147] S. A. Kovalenko, A. L. Dobryakov, J. Ruthmann, N. P. Ernsting, Femtosecond spectroscopy of condensed phases with chirped supercontinuum probing, Phys Rev A: At , Mol Opt Phys 59 (1999) 2369-2384.

[148] S. J. Rosenthal, X. L. Xie, M. Du, G. R. Fleming, Femtosecond solvation dynamics in acetonitrile - observation of the inertial contribution to the solvent response, J Chem Phys 95 (1991) 4715-4718.

[149] P. J. Rossky, J. D. Simon, Dynamics of chemical processes in polar-solvents, Nature 370 (1994) 263-269.

[150] G. R. Fleming, M. H. Cho, Chromophore-solvent dynamics, Annu Rev Phys Chem 47 (1996) 109-134.

[151] M. L. Horng, J. A. Gardecki, A. Papazyan, M. Maroncelli, Subpicosecond measurements of polar solvation dynamics: Coumarin 153 revisited, J Phys Chem 99 (1995) 17311-17337.

[152] J. Demas, G. Crosby, The measurement of photoluminescence quantum yields. a review., J Phys Chem 75 (1971) 991-1024.

[153] J. Lakowicz, Chapter 5. frequency-domain lifetime measurements, in: Principles of Fluorescence Spectroscopy, Kluwer Academic and Plenum Publishers, New York, 1999.

[154] H. Abramczyk, I. Szymczyk, Aggregation of phthalocyanine derivatives in liquid solutions and human blood, J Mol Liq 110 (2004) 51-56.

[155] J. W. Owens, M. Robins, Phthalocyanine photophysics and photosensitizer efficiency on human embryonic lung fibroblasts, J Porphyrins Phthalocyanines 5 (2001) 460-464.

[156] K. A. Nguyen, R. Pachter, Ground state electronic structures and spectra of zinc complexes of porphyrin, tetraazaporphyrin, tetrabenzoporphyrin, and phthalocyanine: A density functional theory study, J Chem Phys 114 (2001) 1075710767.

[157] G. Ricciardi, A. Rosa, E. J. Baerends, Ground and excited states of zinc phthalocyanine studied by density functional methods, J Phys Chem A 105 (2001) 5242-5254. 
[158] M. Shapiro, P. Brumer, Principles of the Quantum Control of Molecular Processes, Wiley, New York, 2003.

[159] J. L. Herek, Coherent control of photochemical and photobiological processes, J Photochem Photobiol Chem 180 (2006) 225.

[160] D. Meshulach, Y. Silberberg, Coherent quantum control of multiphoton transitions by shaped ultrashort optical pulses, Phys Rev A: At , Mol Opt Phys 60 (1999) 1287-1292.

[161] N. Dudovich, B. Dayan, S. M. G. Faeder, Y. Silberberg, Transform-limited pulses are not optimal for resonant multiphoton transitions, Phys Rev Lett 86 (2001) 47-50.

[162] S. Mukamel, Principles of Nonlinear Optical Spectroscopy, Oxford University Press, 1995.

[163] R. W. Boyd, Nonlinear optics, Elsevier, 2003.

[164] G. Gale, M. Cavallari, T. Driscoll, F. Hache, Sub-20-fs tunable pulses in the visible from an 82-MHz optical parametric oscillator, Opt Lett 20 (1995) 15621564. 



\section{Summary}

\section{Coherent control of biomolecules}

This thesis presents new strategies for optimal control experiments and beyond. Divided in two parts, it proceeds by first describing the methodological developments and then moves to the studies on functional molecules for artificial photosynthesis and photomedical therapies, demonstrating the power of the chosen approach for complex systems.

The dream of laser control of chemistry has inspired science ever since the advent of the laser. Traditionally, the approach to obtain chemical control has been passive, utilising macroscopic entities like pressure, temperature etc., but recently active methods based on manipulating microscopic properties of the light-matter interaction have emerged. The general goal is to find laser pulse shapes that will drive reactions to desired products and avoid unwanted products. However, the complex molecular world is still a big challenge for theory and accurate predictions of control pulse shapes are not available. To answer this call, an experimental technique involving adaptive femtosecond pulse shaping in a learning loop (see Figure A) was proposed in 1992, and soon followed by numerous successful applications.

In this work we make use of the versatility of this learning-loop technique to study biologically-relevant functional molecules. Our approach is top-down: rather than using simple model systems, we study real molecular systems in the condensed phase. We use the means of laser control as a novel spectroscopic tool to unravel functionally relevant information of the molecular systems. In other words, our aim is not only to control, but to understand the control and to extract new information from it. Since the laser control relies on the quantum-mechanical details of molecular systems we call this quantum control spectroscopy.

The first part of the book discusses challenges and opportunities that follow the use of such a black box approach. In Chapter 2 we describe the methodology and the existing techniques involved. Chapters 4 and 5 present new work concerning the methodological aspects of coherent control. First we focus on improving the blackbox tool by comparing two evolutionary algorithms and showing how well they func- 
tion in solving a real-life physical problem. Second, we think beyond the loop and show how the obtained control results can be utilised to gain further knowledge on the systems.

In the second part we move to the molecular applications. In Chapter 6 we describe the photophysics of an artificial light-harvesting complex in detail and provide a clear temporal and spectral picture of the different energy levels and pathways involved. Chapter 7 presents coherent control results on the energy transfer process taking place in the biomimicking light harvester (see Figure B, panel a). Furthermore, we perform quantum control spectroscopy which leads to a deeper understanding of the control mechanism and sheds light to the functionally relevant motions that influence the efficiency of the energy transfer process.

Chapter 8 is on a model photosensitiser ZnPc for emerging photomedical applications like photodynamic therapy. Relatively little is known about the molecular level photophysics of photosensitisers for these applications. Thus, we characterise the photosensitiser thoroughly in ultrafast transient absorption studies and then use the gained knowledge in subsequent coherent control experiments. Our control target is to enhance the triplet yield by improving the efficiency of the intersystem crossing process that is an essential step in Type 2 reaction path of photodynamic therapy (see Figure B, panel b). After demonstrating control in closed-loop experiments we conduct open-loop control experiments, which lead to a proposed mechanism for the enhancement of the efficiency of the model photodrug.

In conclusion we have built a state-ot-the-art laboratory, developed methodologies and applied them to realise coherent control of biomolecules. The power of the used tools in bringing new insights to photophysics of complex systems is demonstrated. From characterisation using transient absorption spectroscopy via the learning loop to performing quantum control spectroscopy is a long path, but we have demonstrated here that this approach is capable of providing information that is hidden from traditional techniques. Ultimately, these teachings may lead to design principles for chemical engineering as well as practical applications of shaped laser pulses, which makes our approach increasingly appealing.

\section{Janne Savolainen}




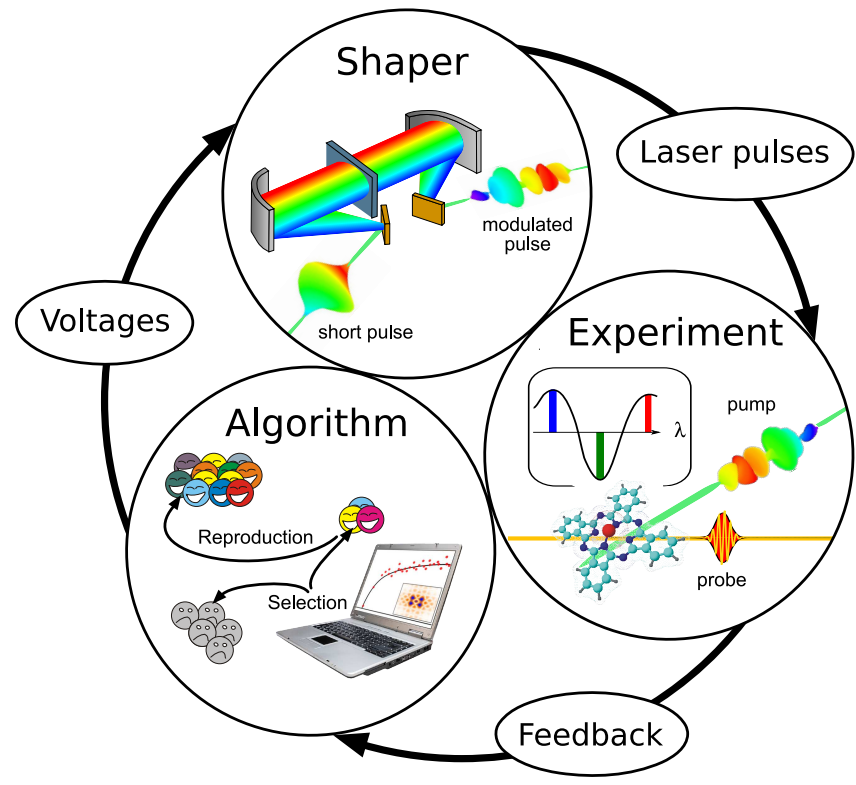

Figure A: The learning-loop experiment

Phase and amplitude patterns (described in Voltages) from the evolutionary algorithm are transformed into pulse shapes in the pulse shaper that modulates the spectral components of short input pulses to produce modulated pulses. The shaped pulses are then used as pump pulses together with spectrally broad probe pulses in a transient absorption experiment, which provides spectrally resolved molecular feedback. According to the feedback the algorithm creates new pulse shapes using operations based on evolutionary principles. A new round can now begin and the loop is let to run until the experiment has converged to the chosen molecular target objective.

Figuur A: Het leerlus experiment

Fase en amplitude maskers (uitgedrukt in voltages) gegeven door het evolutionaire algoritme worden omgezet in pulsvormen door de vormer, die de spectrale componenten van een korte ingangspuls moduleert om in tijd gemoduleerde pulsen te verkrijgen. De gevormde pulsen worden dan gebruikt als pomp-pulsen, samen met een spectraal brede probe-puls in een overgankelijke absorptie experiment. Dit experiment geeft spectrale feedback van het molecuul. Gebaseerd op deze feedback genereert het algoritme nieuwe pulsvormen volgens evolutionaire principes. Een nieuwe ronde kan nu beginnen. De lus wordt doorlopen tot dat het experiment is geconvergeerd op het gekozen moleculaire doel. 
Figura A: O laço de aprendizagem

Os padrões de fase e amplitude (descrito em Voltagens) do algoritmo evolutivo são transformados em impulsos modelados no modelador de impulsos. Os impulsos modelados espectralmente são então utilizados como impulsos de excitação juntamente com impulsos de prova espectralmente amplos, numa experiência de excitação e de prova. Dependendo do feedback experimental, o algoritmo cria então impulsos com novos perfis, usando operações baseadas em princípios evolutivos. Um novo ciclo é iniciado e o processo repete-se até que a experiência convirga para o objectivo definido.

\section{Kuva A: Oppimisluuppikoe}

Evolutionaarisen algoritmin tuottamat vaihe-ja amplitudikuviot (kuvattu jännitteinä) muunnetaan pulssinmuodoiksi pulssinmuokaajassa, joka moduloi lyhyen sisäänmenopulssin spektrikomponentteja muokattujen pulssien tuottamiseksi. Näitä muokattuja pulsseja käytetään sitten pumppupulsseina pumppaa ja koeta -kokeessa yhdessä erittäin laajaspektristen koetinpulssien kanssa. Koe tarjoaa spektraalisesti eritellyn molekyylisen palautteen, jonka mukaisesti algoritmi luo uusia pulssinmuotoja käyttäen evolutionaarisiin periaatteisiin perustuvia periaatteita. Uusi kierros voi nyt alkaa ja oppimisluupin annetaan pyöriä kunnes koe on tarpeeksi lähellä valittua molekyylistä tavoittetta. 
a

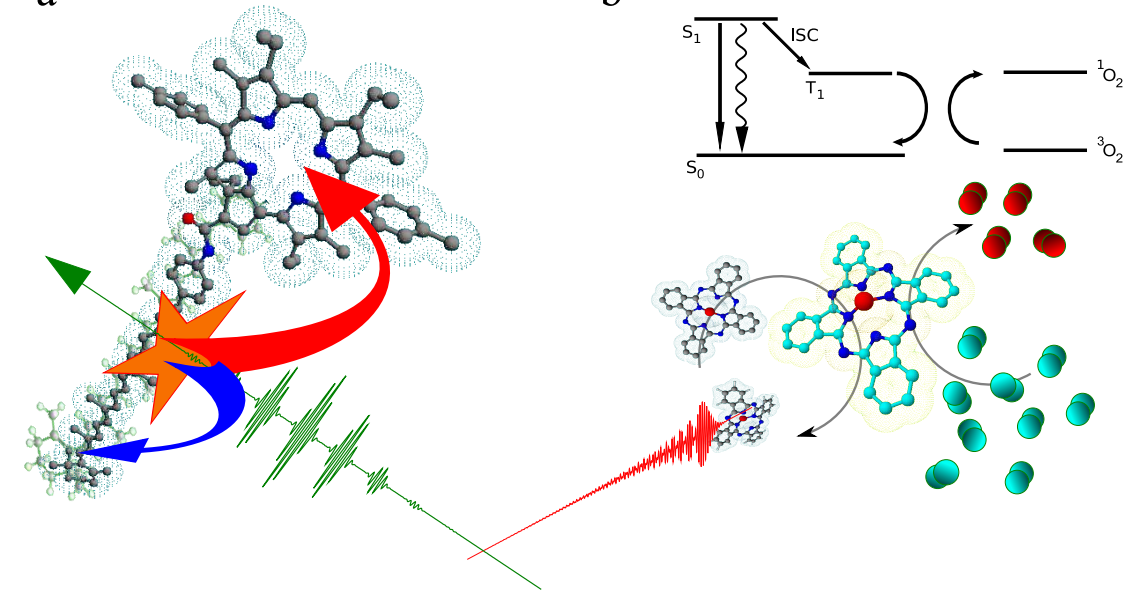

Figure B: Molecular applications

Panel a: We use shaped laser pulses to control the ratio between the energy transfer (red arrow) and internal conversion (blue arrow) processes that take place in the artificial light-harvesting complex. Panel b: In photodynamic therapy, photoexcited sensitiser molecules go through an intersystem crossing (ISC) process to the triplet state (big blue $\mathrm{ZnPc}$ structure) that trigger the formation of singlet oxygen (red molecules), which is highly reactive and causes cell death. We use pulse shaping to find pulses that improve the functional triplet formation over the competing loss channels.

Figuur B: Moleculaire toepassingen

Paneel a: We gebruiken gevormde laser pulsen om de verhouding tussen energieoverdrachts (rode pijl) en interne conversie (blauwe pijl) processen die voorkomen in een kunstmatig licht-opvang-complex te sturen. Paneel b: In fotodynamische therapie gaan gefotoexciteerde moleculen over in de 'triplet' toestand (grote blauwe $\mathrm{ZnPc}$ structuur) van waaruit de formatie van 'singlet' zuurstof (rode moleculen) wordt gestart. Zuurstof in deze toestand is erg reactief en zorgt ervoor dat de cel sterft. We gebruiken de leerlus om pulsvormen te vinden die de formatie van de 'triplet' toestand verbeteren ten opzichte van concurrerende verlies kanalen.

Figura B: Aplicações moleculares

Esquema a: Usamos impulsos LASER modulados para controlar a razão entre os processos de transferência de energia (indicados pela seta vermelha) e de conversão interna (seta azul) que tem lugar no complexo de colheita artificial de luz. Esquema b: $\mathrm{Na}$ terapia fotodinâmica, as moléculas sensibilizadoras fotoexcitadas passam ao estado tripleto (representado pela estrutura de $\mathrm{ZnPC}$ em azul) por um processo the cruzamento singuleto-tripleto. Este estado tripleto dá origem á formação de oxigénio singuleto (moléculas em vermelho), altamente reactivo e letal para as células. Nós 
usamos a modelação de impulsos LASER para encontrar impulsos cujos perfis promovam a formação de $\mathrm{ZnPC}$ no estado tripleto relativamente aos mecanismos alternativos.

\section{Kuva B: Molekyylisovellukset}

Paneeli a: Käytämme muokattuja laserpulsseja keinotekoisessa valonsieppauskompleksissa tapahtuvan energiansiirtoprosessin (punainen nuoli) ja sisäisen konversion (sininen nuoli) välisen haarautumiskertoimen kontrolloimiseen. Paneeli b: Fotodynaamisessa terapiassa viritetyt valoherkistäjämolekyylit käyvät läpi spinkielletyn konversion (intersystem crossing, ISC) triplettitilalle, jotka avaavat singlettitilaisten happimolekyylien (punaiset molekyylit) tuoton. Singlettihappi on erittäin reaktiivinen ja johtaa solun kuolemaan riittävinä pitoisuuksina. Käytämme tässä väitöskirjassa pulssinmuokkausta pulssien, jotka parantavat toiminnallisen triplettikanavan tehokkuutta, löytämiseen. 


\section{Samenvatting}

\section{Koherente sturing van biomoleculen}

Dit proefschrift beschrijft nieuwe strategieën voor experimenten met optimale sturing en resultaten van de toepassing hiervan. In het eerste deel wordt de ontwikkeling van deze methoden beschreven, waarna het proefschrift verder gaat over de toepassing op functionele moleculen voor kunstmatige fotosynthese en fotomedische therapieën. Deze toepassingen demonstreren de kracht van de gekozen methoden voor de studie van complexe systemen.

De droom om chemische reacties te besturen met behulp van licht heeft de wetenschap geïnspireerd sinds de uitvinding van de laser. Traditioneel wordt in de chemie de controle passief aangepakt, veelal microscopische eigenschappen zoals druk en temperatuur worden gebruikt. Meer recent zijn er ook actieve controle mechanismen ontwikkeld, gebaseerd op de microscopische interactie van licht met de materie. In het algemeen is het doel om een laser pulsvorm te vinden die de reactie naar het gewenste product stuurt, terwijl de vorming van ongewilde bijproducten vermeden wordt. De complexe wereld van moleculen is nog steeds een grote uitdaging voor theorie en nauwkeurige voorspellingen van de benodigde pulsvormen zijn niet te maken. Als mogelijk antwoord op dit probleem werd in 1992 een methode gebaseerd op een flexibele pulsvormen in een leerlus (zie Figuur A) voorgesteld en al snel volgden vele succesvolle toepassingen van deze techniek.

In het werk dat voor u ligt maken we gebruik van de veelzijdige toepasbaarheid van de leerlus methode om biologisch relevante moleculen te bestuderen. In plaats van simpele modelsystemen bestuderen we echte moleculaire systemen in oplossingen, de omgeving waarin deze moleculen functioneren. We gebruiken de middelen van laser sturing als een nieuwe spectroscopische methode om informatie relevant voor de werking van deze moleculaire systemen te achterhalen. Omdat de sturing afhangt van de microscopische details van de moleculaire kwantum systemen, kunnen we zeggen dat we 'Kwantum Sturing Spectroscopie' uitvoeren. Dus, het doel is niet sturing op zich, maar om nieuwe fysica uit de sturing te halen.

Het eerste deel van dit boek beschrijft de moeilijkheden en mogelijkheden ver- 
bonden aan het gebruik van deze 'zwarte doos' aanpak. In het tweede hoofdstuk beschrijven we de methode en al bestaande technieken. De hoofdstukken vier en vijf presenteren nieuw ontwikkelde methoden betrekking hebbende op coherente sturing. Eerste focussen we op het verbeteren van de zwarte doos aanpak door het vergelijken van twee evolutionaire algoritmen. We laten zien hoe deze algoritmen functioneren in de aanpak van een echt fysisch probleem. Ten tweede denken we verder dan de lus en laten zien hoe de behaalde resultaten gebruikt kunnen worden om nieuwe kennis over het systeem te verkrijgen.

In deel twee van het boek stappen we over naar toepassingen op moleculaire systemen. In hoofdstuk zes wordt de foto-fysica van een artificieel licht-opvang-complex in detail beschreven. Een duidelijk beeld van de spectra en dynamica wordt gegeven door door het toekennen van de verschillende toestanden en de wegen waarin ze verbonden zijn. Hoofdstuk zeven presenteert de resultaten met coherente sturing van het energie overdracht proces in het artificiële complex (zie Figuur B, paneel a). Verder doen we kwantum sturing spectroscopie die leidt tot een dieper begrip van het sturingsmechanisme en belichten de relevante bewegingen die de functionaliteit van het energie overdracht proces beïnvloeden.

Hoofdstuk acht gaat over een model fotogevoelig medicijn, $\mathrm{ZnPc}$, dat toegepast wordt in nieuwe fotomedische therapieën. Relatief weinig is bekend over de fotofysica van fotogevoelige moleculen in deze toepassingen. Eerst wordt het molecuul dus grondig gekarakteriseerd in een overgankelijke absorptie studie, waarna we deze kennis gebruiken in een sturingsexperiment. Ons sturingsdoel is het vergroten van de opbrengst in de 'triplet' toestand door de efficiëntie van de overgang naar deze toestand te verhogen. Dit proces is een essentiële stap in het type 2 reactie pad van fotodynamische therapie (zie Figuur B, paneel b). Na demonstratie van sturing in een gesloten lus experiment voeren we open-lus experimenten uit die leiden tot een voorstelling van een mechanisme voor de verhoging van de efficiëntie van het fotomedicijn.

Concluderend, een laboratorium volgens de modernste technieken is door ons opgebouwd. Nieuwe methoden zijn ontwikkeld en toegepast om coherente sturing op bio-moleculen te bereiken. De kracht van de nieuwe methoden om nieuwe inzichten te geven in de foto-fysica van complexe systemen is gedemonstreerd. Van karakterisatie in overgankelijke absorptie spectroscopie via de leerlus naar kwantum sturing spectroscopie is een lange weg, maar we hebben laten zien dat deze weg kan leiden tot nieuwe informatie die verborgen blijft voor traditionele technieken. Uiteindelijk kunnen deze lessen leiden tot principes voor chemische ontwikkeling als ook praktische toepassingen van gevormde laser pulsen. Dit maakt de door ons ingeslagen richting meer en meer aantrekkelijk. 


\section{Resumo}

\section{Controle coerente de biomoléculas}

Esta tese introduz e aplica novas estratégias de controle coerente. Na primeira de duas partes é apresentado o desenvolvimento da metodologia e na segunda descrito o estudo de moléculas activas em processos fotosintéticos ou na fototerapia médica demonstrando o potencial da aplicação dos métodos desenvolvidos a sistemas complexos.

O sonho de controlar reacções químicas utilizando o LASER tem inspirado a ciência desde o advento do mesmo. Tradicionalmente, o controle químico é feito de forma passiva, variando parâmetros macroscópicos como a temperatura e a pressão. No entanto surgiram recentemente métodos de controle activos com base nas propriedades da interacção entre luz e a matéria. O objectivo geral é de encontrar impulsos LASER cujas formas sejam capaz de controlar reaç̧ões promovendo a formação de compostos alvo e diminuindo a formação de produtos secundários indesejados. No entanto o complexo mundo molecular é ainda um grando desafio para a teoria, impossibilitando desta forma o cálculo de impulsos de controle. Como resposta a este desafio uma técnica experimental de modelação adaptável de impulsos de femtosegundos baseado num laço de aprendizagem (ver Figura A) foi proposta em 1992 encontrando logo após numerosas aplicações.

$\mathrm{O}$ trabalho aqui apresentado faz uso da versatilidade deste laço de aprendizagem adaptável no estudo de moléculas de importância biológica. A nossa aproximação é top-down, i.e. em vez de usar um sistema modelo simple, estudamos sistemas moleculares complexos reais em fase condensada. O controle coerente é aqui utilizado como uma nova ferramenta espectroscópica para obter e resolver informação sobre a funcionalidade do sistema molecular em estudo. Por outras palavras fazemos espectroscopia de controle quântico. O nosso objectivo é, não somente o controle mas também uma melhor compreensão do processo de controle.

$\mathrm{Na}$ primeira parte da tese discutem-se os desafios e oportunidades intrínsecos a esta aproximação black-box. No capítulo 2 é descrita a metodologia usada e a tecnologia existente. No capítulo 4 e 5 apresentam-se novos resultados no que diz respeito à 
metodologia de controle coerente. Primeiramente concentramo-nos sobre o melhoramento da ferramenta black-box através da comparação de dois algoritmos evolutivos e demonstrando a sua eficácia na resolução de um problema real específico.

A segunda parte da tese é dedicada à aplicação a sistemas moleculares. No capítulo 6 descreve-se em pormenor a fotofísica de um complexo de colheita artificial de luz bem como os diferentes níveis de energia e mecanismos involvidos. No capítulo 7 apresentam-se os resultados de controle coerente de processos de transferência de energia que ocorrem no complexo biomímico da colheita artificial de luz (ver Figura B, Esquema a). Em seguida é feita a espectroscopia de controle quântico que permite um conhecimento mais aprofundado do mecanismo de controle e esclarece os processos que influenciam a eficiência da transferência de energia.

O capítulo 8 é dedicado ao modelo de fotosensibilizador $\mathrm{ZnPC}$ com aplicação em fototerapias como por exemplo a terapia fotodinâmica. No contexto destas aplicações terapêuticas, pouco se sabe ainda sobre os processos fotofísicos que envolvem fotosensibilizadores a nível molecular. Por esta razão procuramos aqui caracterizar pormenorisadamente a função destes fotosensibilizadores através do estudo das absorções transientes ultrarápidas e da aplicação do conhecimento adquirido em subsequentes experiências de controle coerente. O objectivo é de aumentar o rendimento quântico do estado tripleto através do melhoramento da eficiência do cruzamento singuleto ttripleto, passo essencial no mecanismo tipo 2 da terapia fotodinâmica (ver Figura B, Esquema b). Uma vez demonstrado o controle em experiências de laço de aprendizagem fechado passa-se então a experiências de laço de aprendizagem aberto que permitem a proposta de um mecanismo para o melhoramento da eficiência do fotofármaco modelo.

Concluindo, construímos um laboratório que reflecte o mais alto nível de conhecimento actual, desenvolvemos metodologias e aplicámo-las ao controle coerente de biomoléculas, demonstrando assim a capacidade das técnicas utilizadas na compreensão da fotofísica de sistemas complexos. O percurso que vai da caracterização dos sistemas através de espectroscopia de absorção transiente pelo laço de aprendizagem até à espectrocopia de controle quântico é longo, mas conseguimos aqui demostrar que a nossa aproximação fornece informação que não é acessível pelos técnicas tradicionais. Os métodos aqui apresentados poderão eventualmente levar ao desenvolvimento de novos princípios no campo da engenharia química, bem como a aplicações práticas da modelação de impulsos LASER, tornando a aproximação aqui proposta bastante atraente.

\section{Janne Savolainen}




\section{Yhteenveto}

\section{Biomolekyylien koherentti kontrollointi}

Tämä väitöskirja käsittelee uusia strategioita molekyylien optimaalista kontrollointia varten. Kirja on jaettu kahteen osaan ja etenee kuvaten ensin saavutetut metodologiset edistysaskeleet joita seuraa niiden soveltaminen toiminnallisten molekyylien kontrollointiin. Valitun lähestymistavan tehokkuus monimutkaisten biologisten systeemien tutkimuksessa havainnollistetaan keinotekoisen valonsieppaajakompleksin sekä fotodynaamisen lääkemolekyylin avulla.

Unelma kemian kontrolloimisesta laservalon avulla on inspiroinut tiedettä laserin keksimisestä lähtien. Perinteisesti kemiallinen kontrolli on ollut passiivista, käyttäen hyväkseen makroskopisia suureita kuten painetta, lämpötilaa jne., mutta nyt on alkanut ilmetä uusia aktiivisia menetelmiä jotka perustuvat valon ja aineen vuorovaikutusten manipuloimiseen. Laserkontrollin yleinen tavoite on löytää sellaisia laserpulssin muotoja, jotka ajavat reaktiota kohti haluttuja tuotteita, samalla välttäen ei-haluttujen sivutuotteiteiden muodostumista. Molekyylien monimutkainen maailma on kuitenkin vielä suuri haaste teoreettisille menetelmille, joten tarkkoja ennustuksia tarvittavista pulssinmuodoista ei yleensä ole saatavilla. Tämän ongelman ratkaisemiseksi esiteltiin 1992 kokeellinen menetelmä, joka perustuu femtosekuntipulssien mukatutuvaan muokkaamiseen oppimisluupissa (katso Kuva A) ja jota pian seurasivat lukuisat onnistuneet sovellukset.

Tässä väitöskirjatyössä hyödynnetään oppimisluuppi-tekniikan monikäyttöisyyttä biologisesti tärkeiden toiminnallisten molekyylien tutkimisessa. Lähestymistapamme on 'top-down': Sen sijaan että käyttäisimme yksinkertaistettuja mallisysteemejä me tutkimme varsinaisia toiminnallisia molekyylejä nestefaasissa. Me käytämme laserkontrollimenetelmiä uutena spektroskooppisena työkaluna molekyylien toiminnalle tärkeiden ominaisuuksien selvittämiseksi. Toisin sanoen tavoitteenamme ei ole ainoastaan kontrollointi, vaan myös kontrollin ymmärtäminen ja uuden informaation uuttaminen saavutetuista tuloksista. Koska laserkontrolli on riippuvainen molekyylien kvanttimekaanisista yksityiskohdista me kutsumme tätä lähestymistapaa kvanttikontrollispektroskopiaksi. 
Tämän kirjan ensimmäinen osa käsittelee haasteita ja mahdollisuuksia jotka liittyvät edellämainitun ‘black-box' lähestymistavan käyttöön. Luvussa 2 kuvataan käytetty metodiikka ja olemassa olevat tekniikat. Luvut 4 ja 5 esittävät koherenttiin kontrollointiin liittyviä uusia metodologisia kehitysaskelia. Ensin keskitymme 'black-box' -työkalun kehittämiseen vertailemalla kahta evolutionaarista algoritmia sekä esittämällä kuinka ne suoriutuvat tosielämän fysikaalisen ongelman ratkaisemisesta. Tämän jälkeen katsomme oppimisluupin ulkopuolelle ja näytämme kuinka saavutettuja optimaalisen kontrollin tuloksia voidaan käyttää systeemien edelleen tutkimisessa.

Kirjan toisessa osassa siirrymme käsittelemään kontrollimenetelmien sovelluksia molekyyleihin. Luvussa 6 selvitämme keinotekoisen valonsieppaajakompleksin fotofysiikkaa yksityiskohtaisesti sekä annamme selkeän aika- ja spektrikuvauksen eri energiatasoista ja niihin liittyvistä energiavirtapoluista. Luvussa 7 esitämme keinotekoisessa valonsieppauskompleksissa tapahtuvan energiansiirtoprosessin koherenttia kontrollointia (katso Kuva B, paneeli a). Tämän lisäksi suoritamme kvanttikontrollispektroskopiaa, joka johtaa kontrollitulosten parempaan ymmärtämiseen ja valaisee toiminnallisesti tärkeiden vapausasteiden vaikutuksia energiansiirtoprosessin tehokkuuteen.

Luku 8 käsittelee valoherkistäjämolekyyliä fotolääketieteellisiä sovelluksia varten. Valoherkistäjämolekyylien molekyylitason fotofysiikasta tiedetään tähän asti suhteellisen vähän, joten me ensin karakterisoimme näytemolekyylin huolellisesti pumppaa ja koeta -spektroskopian avulla. Saavutettuja tuloksia hydynnetään tämän jälkeen kontrollikokeissa. Kontrollitavoitteenamme on parantaa tripletti-tilaisen valoherkistäjän saantoa edistämällä spinkiellettyä konversiota, joka on olennainen askel fotodynaamisen terapian tyypin 2 -reaktioketjussa (katso Kuva B, paneeli b). Osoitamme ensin, että optimaalisen kontrollin avulla suhteellista triplettisaantoa voidaan lisätä. Tämän jälkeen suoritamme avoimen luupin kokeita, jotka johtavat ehdotetuun mahdolliseen kontrollimekanismiin tutkitun fotolääkkeen tehokkuuden lisäyksen takana.

Olemme rakentaneet huippulaboratorion, kehittäneet metodiikkaa ja soveltaneet niitä biomolekyylien koherenttiin kontrollointiin, ja näinollen havainnollistaneet käytettyjen työkalujen tehokkuutta uuden tiedon ja oivalluksien tuottamisessa. Tie karakterisoinnista pumppaa ja koeta -kokeen avulla oppimisluupin kautta kvanttikontrollispektroskopiaan on pitkä, mutta tässä väitösirjassa esitetyty tulokset osoittavat, että tämä lähestymistapa pystyy tuottamaan informaatiota, joka on piilossa perinteisiltä tekniikoilta. Viime kädessä nämä opit voivat johtaa uusiin synteettisiin sunnitteluperiaatteisiin kemiallista teollisuutta varten sekä muokattujen laserpulssien käytännöllisiin sovelluksiin, mikä tekee lähestymitavastamme yhä kiinnostavamman. 


\section{Acknowledgements}

OK, it is time to stop staring at the Mediterranean sea, have another sip of sangria, and offload some gratitude. Oh yes, there is quite some to share. It feels a bit of an awkward task to try to do this in couple of pages, since this book and my whole $\mathrm{PhD}$ work has benefited of the ample support of so many.

It has been my great pleasure to perform my $\mathrm{PhD}$ work in the Biomolecular Control group. From the first invitation email to these last hectic moments of writing-up this book, I've had my supervisor's support, attention, and words of advice available. Jennifer, you are without a doubt the best boss I've ever had; I thank you enormously for making sure that the door was always open and that I could approach you with anything; big or small, good or bad. Thank you so much for your patience and for being my supervisor over these turbulent years!

After the first two years of laboratory development together with the coolest group member, Niels (thanks man, it has been super making things work!), we had the pleasure to start sharing the efforts with two splendid gentlemen Riccardo and Peter, and a bit later with Maaike. I think our AMOLF days together with warmth and weird nostalgy as I've already been missing you here in Enschede! A big bow goes also to Dennis and Thomas, the two master students I had an honour to supervise. A significant part of my $\mathrm{PhD}$ project was much involved with technical details and laboratory development crucial for the research, and without the help of Rob, Hinco, Willem, Ed, Ad, Wouter, Silvia, Grace, Wim, Iliya, Dirk-Jan, Duncan, Richard, Rutger, Jan, Lars etc. etc. I would not be sitting here typing these last words into my thesis. Cheers guys, it has been a great pleasure working with you, and thanks for welcoming me so well in AMOLF in the beginning!

I have a massive gratitude to pay for the discussions the data and aid in writing thethe key contributions of this book, the spearheads of quantum control spectroscopy: Jürgen and Tiago with the important support from their professor Marcus Motzkus. I will always be grateful to Marcus for the hospitality during my visits to Garching and Marburg he accommodated as well as the scientific help he provided. Cheers guys, and rock on, as they say! 
My group in the University of Twente: Di, Ali, Jeroen, and Daan and the rest of the OS bunch have all already lent their helping hands and aided in the finalising touches of this book. They've truly been responsive, and afforded many positive inputs. Hence, thanks guys; looking forward to the times ahead. My best goes also to the old Jyväskylä gang, particularly Janne whom I am actually to 'blame' to get invited to AMOLF in the first place. I also want to mention Jouko, Pasi, and Viivi as this path originates from the darkness shared together in the femtolab of the University of Jyväskylä.

I'd also like to thank Lars Wilmes and Thomas Bäck group from LIACS for a really enjoyable collaboration and Ana Moore from Arizona, Jaap and Ben Feringa for providing samples for us. My thanks go also to Misha and Joep for a nice collaboration. I also want to thank my roommates Anna, Joan, Sébastien and Jocelyn for the entertaining company. Further, the AMOLF youth; Yves, Avi, Adriaan, Han-Kwang, Marijn, Dorte, Omair, Tanja, Frederik, Erica, Pavol, Klaas-Jan, Rutger, Marco, Rimco, Basak, Maria, Frank, Simon, Roland, Tarik Lennaert and all; many thanks for all the conversations, outings, PV activities that we shared over the times, in and out of the lab. And, of course, warm thoughts of gratitude are directed toward the insipiring femto-seniors: Huib, Sander, Mischa, and Marc.

At times, the companionship of my family and friends has been well needed and gladly received. From the Amsterdam end of the world, I want to particularly thank Richie for fine times we had in A'dam, Budapest, Romania, and Finland. Similarly, the activities as well as the passivities shared with Joost, Irina, family Kappelhof, Jarmo, Adi, Liam, Ioana, Maarten, Gary, Priscilla, Yvonne, Rebecca, and Sandy have been a engaging and delightful, but also a source of solace when the life in Amsterdam decided it was time to cast the grey spell. It has been wonderful to know that I could always rely on Juha's and Nakki's friendship and willingness to facilitate the holidays in Finland, which provided the needed variety and change of rhythm. Naturally, my parents, funky brothers and their families have been an important part of the Finnish connection during my stay in A'dam. I am so happy for their visits during these years. Especially the visit of my god daughter Vilhelmiina and her older brother Volmari springs to mind. And who could forget the holiday in Portugal! I also want to thank Ester's family, especially Mãe Simões for the great times in Lisbon and Algarve.

And thank you Esterinha for enduring numerous rantings and mood swings, the amplitude of which did sometimes scare even the undersigned. Although, not making any promises to grow up, I intend to have a bit more considerate approach in the future. Girl, we've got such an adventure ahead, thanks for wanting to share it with me!

Barcelona, Janne Savolainen April 2008 

